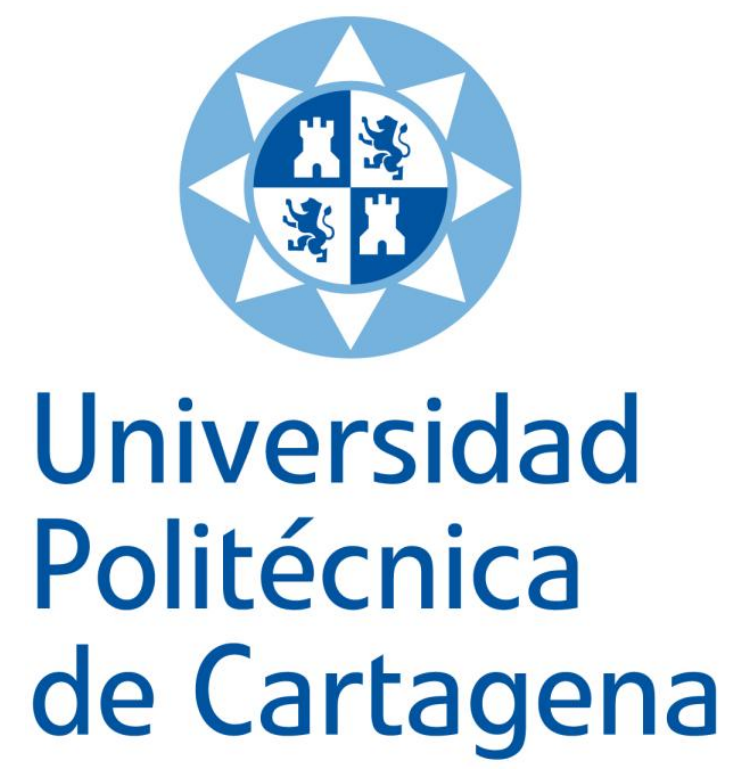

"Caracterización de músculos artificiales con capacidades sensores/actuadores e intercambio mayoritario de cationes"

Laura Luz Valero Conzuelo

2016 


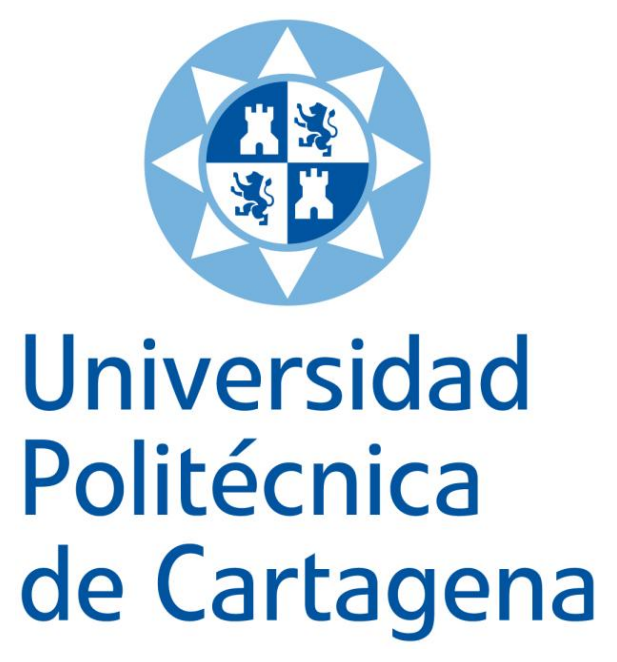

"Caracterización de músculos artificiales con capacidades sensores/actuadores e intercambio mayoritario de cationes"

Laura Luz Valero Conzuelo

Director de la Tesis

Toribio Fernández Otero

Co-Director de la Tesis

José Gabriel Martínez Gil

2016 


\title{
Dedicatorias
}

\begin{abstract}
A Dios:
Por cada amanecer a mi lado.

A mis Padres:

Celia y Enrique por enseñarme el camino de la vida con su ejemplo de amor y dedicación al trabajo.
\end{abstract}

\section{A mis Hijos:}

Por la pertenencia en la fe y el amor

\section{A mi Esposo:}

Por todos los momentos que compartimos

A mis hermanos, mis sobrinos y mi nieto

\begin{abstract}
Al Dr. Toribio:
Por su valiosa asesoría en el desarrollo de esta tesis doctoral. y por sus conocimientos y experiencia aportado a la presente .

Dr. José Gabriel Martínez Gil por su Co-asesoría en esta tesis
\end{abstract}

\section{A todos mis compañeros del CEMI}

Joaquín, María, Mercedes, Inés, Angelita, el buen Franz, Masaki, Yahya, Javi, y Víctor. 


\section{Agradecimientos}

\section{Universidad Politécnica de Cartagena}

Universidad Autónoma del Estado de México:

Facultad de Ingeniería

Centro de Investigación Científica de Yucatán

A los proyectos y Convenios que apoyaron esta tesis doctoral:

Al Gobierno de España Proyecto MAT2008-06702

A la fundación Séneca Proyecto 08684/PI/08

Consejería de Educación de Murcia, Plan Regional de Ciencia y Tecnología

Consejo Nacional de Ciencia y Tecnología

CONACYT Proyecto 47066

Consejo Mexiquense de Ciencia y Tecnología

COMECYT

UAEM-PROMEP 103.5/06/1975 
Los resultados obtenidos durante esta tesis han sido de la suficiente calidad e interés como para dar lugar a la publicación de 7 artículos en revistas internacionales indexadas en el Journal Citations Reports $\AA$ :

1. Laura Valero, Joaquin Arias-Pardilla, Mascha Smit, Juan CauichRodríguez, Toribio F. Otero. Polypyrrole free-standing electrodes sense temperature or current during reaction Polymer International, año 2010, volumen 59, páginas 337-342. (ISI-JCR IF: 2.245, Q1 en instrumentos e instrumentación, Q2 en química, analítica y Q3 en electroquímica).

2. Laura Valero Conzuelo, Joaquin Arias-Pardilla, Juan V. CauichRodríguez, Mascha Afra Smit, Toribio Fernández Otero. Sensing and Tactile Artificial Muscles from Reactive Materials Sensors, año 2010, volumen 10, páginas 2638-2674. (ISI-JCR IF: 2.409, Q2 en ciencia de polímeros).

3. Francisco Garcia-Cordova, Laura Valero, Yahya A. Ismail, Toribio F. Otero. Biomimetic polypyrrole based all three-in-one triple layer sensing actuators exchanging cations Journal of Materials Chemistry, año 2011, volumen 21, páginas 17265-17272. (ISI-JCR IF: 6.626, Q1 en química, física y ciencia de materiales, multidisciplinar).

4. Laura Valero, Joaquín Arias-Pardilla, Juan Cauich-Rodríguez, Mascha A. Smit, Toribio F. Otero. Characterization of the movement of polypyrrole-dodecylbenzenesulfonate-perchlorate/tape artificial muscles. Faradaic control of reactive artificial molecular motors and muscles Electrochimica Acta, año 2011, volume 56, páginas 3721 3726. (ISI-JCR IF: 4.504, Q1 en electroquímica).

5. Laura Valero, Toribio F. Otero, Jose G. Martinez. Exchanged Cations and Water during Reactions in Polypyrrole Macroions from Artificial Muscles Chemphyschem, año 2014, volumen 15, páginas 293-301. (ISI-JCR IF: 3.419, Q1 en física, atómica, molecular y química, Q2 en química, física). 
6. Toribio F. Otero, Jose G. Martinez, Masaki Fuchiwaki, Laura Valero. Structural Electrochemistry from Freestanding Polypyrrole Films: Full Hydrogen Inhibition from Aqueous Solutions Advanced Functional Materials, año 2014, volumen 24, páginas 1265-1274. (ISIJCR IF: 11.805, Q1 en química, multidisciplinar; química, física; ciencia de materiales, multidisciplinar; nanociencia y nanotecnología; física, aplicada; y física, materia condensada).

7. Laura Valero, Jose G. Martinez, Toribio F. Otero. Creeping and structural effects in Faradaic artificial muscles Journal of Solid State Electrochemistry, año 2015, volumen 19, páginas 2683-2689. (ISI-JCR IF: 2.446, Q2 en electroquímica). 
Universidad

Politécnica

'de Cartagena

DT-16

\section{CONFORMIDAD DE SOLICITUD DEAUTORIZACIÓN DE DEPÓSITO DE TESIS DOCTORAL POR EL/LA DIRECTOR/A DE LA TESIS}

D./Da.----Dn. Toribio Fernández Otero-- Director/a de la Tesis doctoral -

\section{INFORMA:}

Que la referida Tesis Doctoral, ha sido realizada por D/D ${ }^{\mathrm{a}}$.Laura Luz Valero Conzuelo

-, dentro del programa de doctorado -"Electroquímica. Ciencia y tecnología", dando mi conformidad para que sea presentada ante la Comisión de Doctorado para ser autorizado su depósito.

La rama de conocimiento en la que esta tesis ha sido desarrollada es:

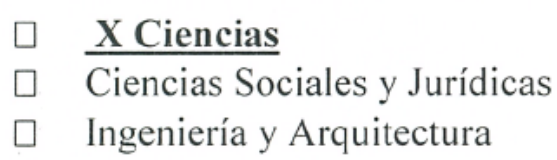

En Cartagena, a 22 de_Septiembre del 2015

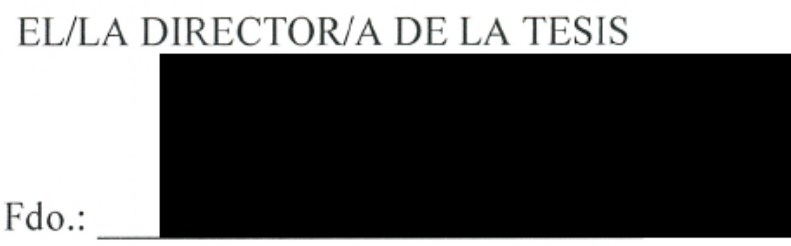

Dr.. Toribio Fernández Otero 


\section{Universidad}

Politécnica

de Cartagena

\section{CONFORMIDAD DE DEPÓSITO DE TESIS DOCTORAL POR LA COMISIÓN ACADÉMICA DEL PROGRAMA}

D/D ${ }^{\mathrm{a}}$. -, Don. Enrique Herrero Rodríguez

Presidente/a de la Comisión Académica del Programa Doctorado en Electroquímica, ciencia y tecnología

\section{INFORMA:}

Que la Tesis Doctoral titulada, ""CARACTERIZACIÓN DE MÚSCULOS ARTIFICIALES CON CAPACIDADES SENSORES/ACTUADORES E INTERCAMBIO MAYORITARIO DE CATIONES" -

-", ha sido realizada, dentro del mencionado programa de doctorado, por D/D ${ }^{\mathrm{a}}$. Laura Luz Valero Conzuelo -, bajo la dirección y supervisión del Dr. Toribio Fernández Otero -y CoDirección: del Dr. José Gabriel Martínez Gil.

En reunión de la Comisión Académica de fecha 25/09/2015, visto que en la misma se acreditan los indicios de calidad correspondientes y la autorización del Director de la misma, se acordó dar la conformidad, con la finalidad de que sea autorizado su depósito por la Comisión de Doctorado.

La Rama de conocimiento por la que esta tesis ha sido desarrollada es:

\section{$\square$ Xiencias}

$\square$ Ciencias Sociales y Jurídicas

$\square$ Ingeniería y Arquitectura

En Alicante, a -25-de -septiembre- de-2015-

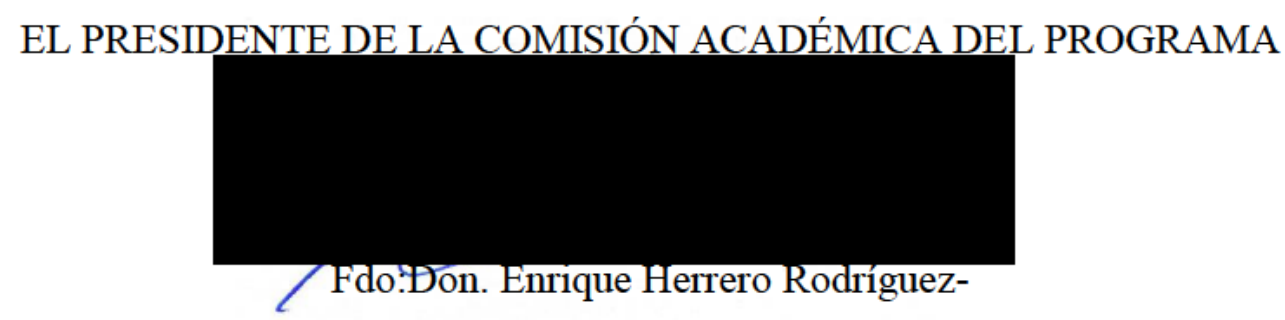




\section{RESUMEN}

Durante la pasada década, se han estado buscando nuevos materiales biomiméticos capaces de imitar el comportamiento de órganos humanos de tal forma que sea posible aplicarlos en diferentes tecnologías tales como: tecnologías de bajo coste, escalables, de bajo consumo de energía y aquellas con importantes potencialidades en campos como la salud, la robótica, los nervios artificiales o los músculos artificiales entre otras. La mayoría de los materiales y dispositivos biomiméticos estudiados imitan la matriz extracellular de los seres vivos y sus funciones físicas.

Ahora, por primera vez, los polímeros conductores, y otros materiales electroactivos intercambian iones y agua durante reacciones electroquímicas: el material se transforma en un gel denso y electroactivo. El contenido de dicho gel y las reacciones que en él ocurren imitan, por primera vez en ciencia, la composición (en su expresión más simple) y las reacciones que ocurren en la matriz intracelular de las células funcionales de los seres vivos. Durante las reacciones electroquímicas (oxidación o reducción), la composición relativa del gel (polímeroion-agua) varía de forma reversible en varios órdenes de magnitud. A la vez que su composición, algunas propiedades que dependen de dicha composición también cambian. La variación reversible del volumen del material impulsada por las reacciones imita el comportamiento de los músculos naturales: músculos artificiales poliméricos o actuadores poliméricos electroquímicos están siendo desarrollados basados en esta propiedad. A la vez que la composición del material, la energía consumida varía en función de las condiciones térmicas, químicas o mecánicas de la reacción. Esto se utiliza para el desarrollo de sensores y biosensores. El volumen y el potencial del material cambian simultáneamente durante la reacción. Aquí exploramos la posibilidad de desarrollar actuadores/sensores: dos elementos trabajando simultáneamente en el mismo dispositivo (físicamente uniforme) imitando el funcionamiento de los músculos hápticos.

En esta tesis se describe la síntesis de películas gruesas de polipirrol/DBS. El comportamiento electroquímico de la película polimérica, utilizada como electrodo autosoportado, es caracterizado asumiendo el intercambio de cationes durante su oxidación/reducción. Para la caracterización electroquímica de películas biomiméticas de polipirrol/DBS, se usan diferentes técnicas electroquímicas y bajo diferentes condiciones experimentales con el fin de comprender la capacidad sensora de la reacciones del material.

El estudio y la caracterización electroquímica del movimiento de bicapas pPy/DBS//cinta corroboran que la reacción está controlada por la expulsión de cationes desde el polímero conductor durante su oxidación y su entrada durante su reducción en el rango completo de potenciales estudiados. El actuador es un dispositivo farádico controlado por la reacción electroquímica origen del movimiento: la velocidad del movimiento angular es una función lineal 
(control sencillo de la velocidad) de la corriente eléctrica aplicada y el ángulo descrito por el movimiento es una función lineal de la carga consumida (también provee otro control sencillo del desplazamiento).

La evolución del potencial muscular y de la energía eléctrica consumida durante la reacción siente las condiciones energéticas de trabajo: energía química (concentración del electrolito), energía térmica (temperatura de trabajo) o energía eléctrica (corriente aplicada). El motor polimérico siente, mientras trabaja, sus condiciones de trabajo. Las curvas de calibración para los diferentes sensores han sido obtenidas.

Se han construido y caracterizado también músculos artificiales tricapa pPy/DBS//cinta//pPy/DBS, corroborando de nuevo el intercambio de cationes durante la reacción, la naturaleza farádica del actuador y la habilidad que tiene de sentir, mientras se mueve, las condiciones en las que trabaja, imitando los músculos hápticos naturales. Las señales actuadoras (corriente y carga) y sensoras (potencial muscular y energía consumida) están presentes simultáneamente en solamente dos hilos de conexión, imitando la comunicación cerebro-músculos.

El estudio de materiales poliméricos con intercambio de cationes y/o aniones abre las puertas al desarrollo de nuevas herramientas y robots blandos, húmedos, biomiméticos y multifuncionales. Señales iónicas, químicas, térmicas y mecánicas pueden ser transformadas en señales eléctricas, transportando información utilizando solamente dos cables conductores, simplificando de esta forma su conexión: el diseño de dispositivos y robots que los contengan anuncia una tecnología más eficiente. 


\section{ABSTRACT}

Over the past decade scientific research has been looking for new biomimetic materials able to imitate human organs behaviour, in such a way that is possible to apply them on different technologies: low cost ones, scalable ones, low energy consumption ones and on those with high potentialities in areas such as health, robotics, artificial nerves and muscles, among others. Most of the studied materials mimic the extracellular matrix (ECM) of living cells and its physical functions.

Now, and for the first time, conducting polymers, and other electroactive materials exchange ions and water through electrochemical reactions: the material becomes a dense electroactive gel. The content of mentioned gel and the reactions happening in it mimic, by the first time in the history of science, the composition (in its simplest expression) and reactions taking place in the reactive intracellular matrix of the functional cells of living beings. During the chemical reactions (oxidation or reduction) the gel relative composition (polymer-ion-water) shifts, in a reversible way, by several orders of magnitude. Along with it several composition-dependent properties of the material change simultaneously. The reversible variation of the material volume driven by the reactions mimics the natural muscles behaviour: artificial polymeric muscles, or polymeric electrochemical actuators, based on this property are being developed. With the material composition the consumed energy change as a function thermal, chemical or mechanical conditions. This fact is used for the development of sensors and biosensors. The material volume and the material potential shift, simultaneously, during the reaction. Here the possibility to develop dual sensing-actuators is explored: two elements working concurrently in the same, physically uniform, device mimicking haptic muscles.

In this thesis the electrochemical synthesis of thick polypyrrole/DBS films is described. The electrochemical behaviour of the polymer film, used as a self-supported electrode, is characterized assuming the exchange of cations during its oxidation/reduction. For the electrochemical characterization of biomimetic films of polypyrrole/DBS, different electrochemical techniques are used and under different experimental conditions with the view to understanding the sensing potentialities of the material reactions.

The study and electrochemical characterization of the motion of pPy/DBS//tape bilayer bending actuators corroborates that the reaction is driven by the expulsion of cations from the conducting polymer to the electrolyte during oxidation and its entrance during reduction, in the full potential range studied. The actuator is a faradaic device controlled by the electrochemical reaction driving the movement: the rate of the angular movement is a linear function (easy control of the velocity) of the applied current and the described angle by the displacement is a linear function of the consumed charge (it also provides another easy control of the displacement) 
The evolution of the muscle potential and that of the consumed electrical energy during the reaction senses the energetic working conditions: chemical energy (electrolyte concentration), thermal energy (working temperature) or electric energy (applied current). The polymeric motor senses, while working, environmental conditions. The sensing calibration curves were attained for the different sensors.

They have been constructed and characterized triple-layer artificial muscles $\mathrm{pPy} / \mathrm{DBS} / / \mathrm{Tape} / / \mathrm{pPy} / \mathrm{DBS}$, corroborating again the exchange of cations during the reaction, the faradic nature of the device and the ability of the device to sense, while moving, its environmental working conditions mimicking natural haptic muscles. The actuator (current and charge) and sensing (muscle potential and involved energy) signals are simultaneously present in only two connecting wires, mimicking brain-muscle intercommunication.

The study of polymeric materials with cationic and/or ionic exchange opens the possibility of working in a future, using also anion-exchange materials, to develop new soft, wet, biomimetic and multifunctional tools and robots. Ionic, chemical, thermal and mechanical signals can be transformed into electrical ones and the involved information is transported using just two wires, simplifying in that way their connection to computers: the design of devices and robots having them heralds a more efficient technology. 


\section{ÍNDICE}

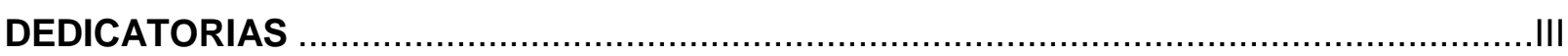

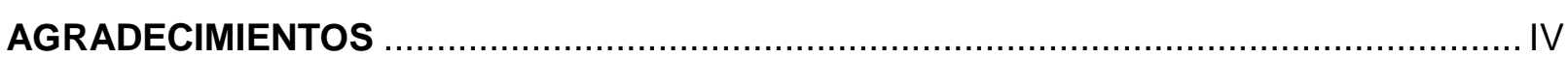

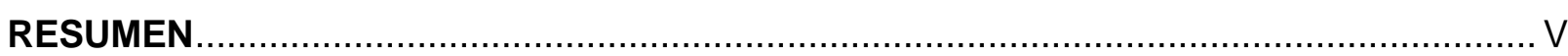

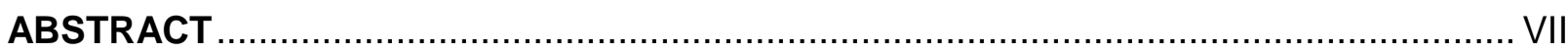

ÍNDICE

ÍNDICE DE FIGURAS.

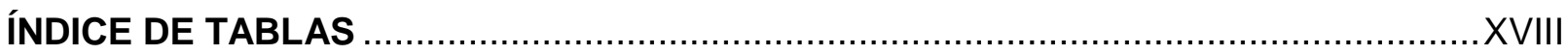

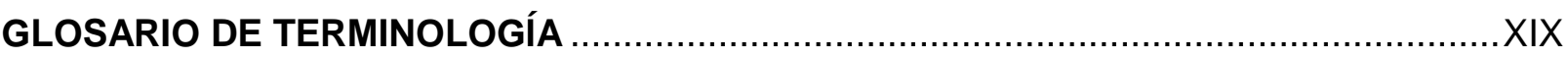

\section{CAPÍTULO I INTRODUCCIÓN}

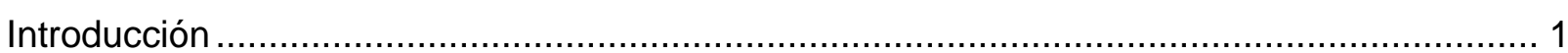

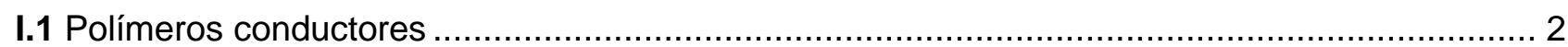

I.2 Clasificación de polímeros conductores ........................................................................ 5

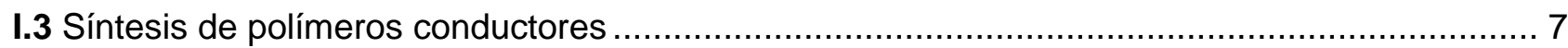

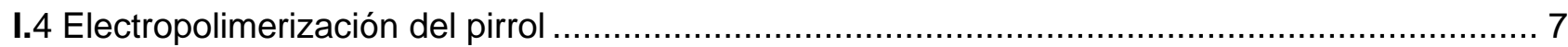

I.5 Oxidación y reducción de Polímeros conductores......................................................... 7

I.6 Propiedades electroquímicas de los polímeros conductores ............................................... 9

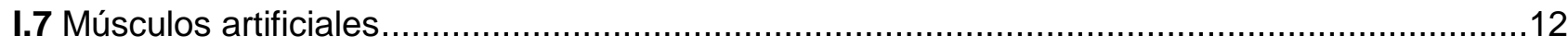

I.7.1 Clasificación de los músculos artificiales (actuadores poliméricos electroquímicos) ..........13

I.8 Propiedades actuadoras/Sensoras de los Polímeros conductores .......................................17

I.9 Potencialidades de los Polímeros Conductores ................................................................17

\section{CAPITULO II METODOLOGIA EXPERIMENTAL}

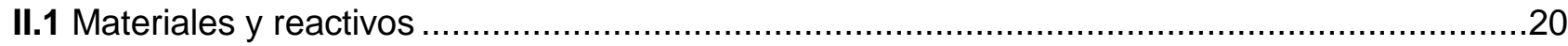

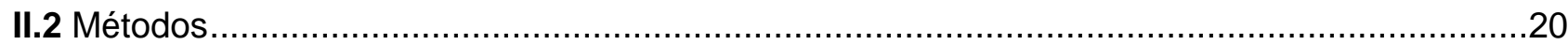

II.2.1 Preparación del sustrato de polimerización .................................................................20

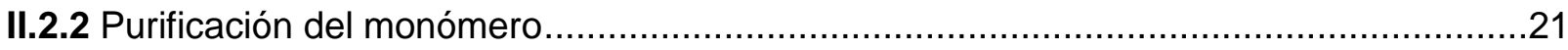

II.2.3 Electrogeneración de películas poliméricas de base pirrol .......................................21

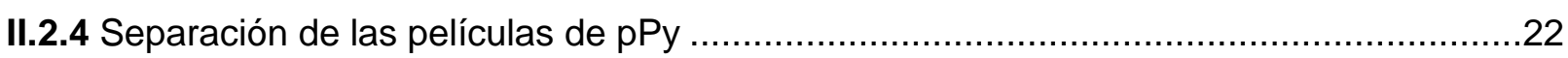

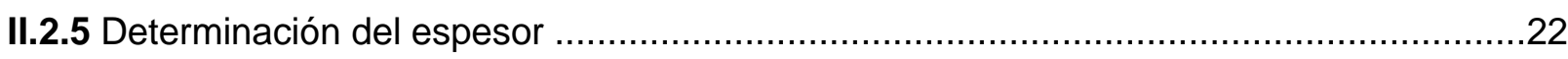

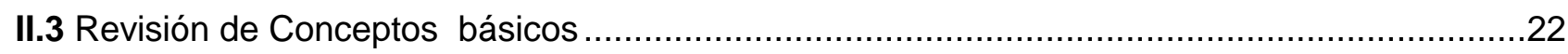

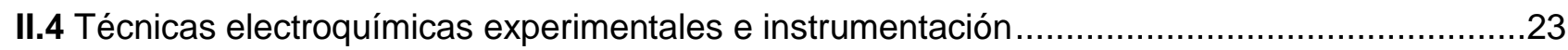

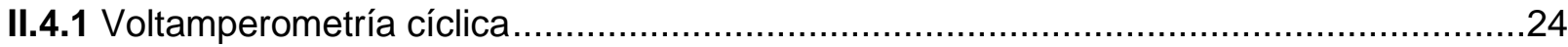


II.4.2 Cronopotenciometría y Cronoamperometría .24

II.5 Electrodos poliméricos autosoportados .25

II.5.1 Valoración del estado de las películas o actuadores caracterizados .26

II.6 Construcción del actuador bicapa: película//cinta adhesiva ............................................27

II.7 Construcción del actuador tricapa pPy/DBS//cinta// pPy/DBS .........................................28

II.8 Caracterización dinámica de los actuadores bicapa y tricapa ..............................................29

\section{CAPITULO III RESULTADOS Y DISCUSIONES}

III.1 Caracterización electroquímica Película pPy/DBS autosoportadas......................................30

III.2 Influencia del límite catódico de potencial .......................................................................30

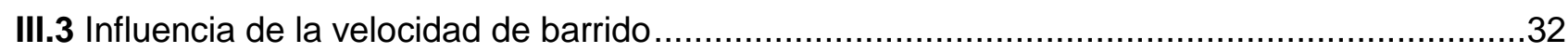

III.4 Reversibilidad, estabilidad en películas autosoportadas pPy/DBS .....................................34

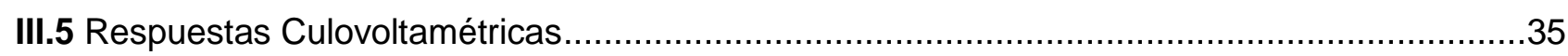

III. 6 Pérdida de electroactividad en películas autosoportadas pPy/DBS (tiempo de vida) ............38

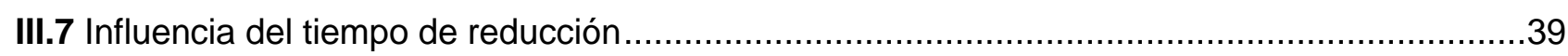

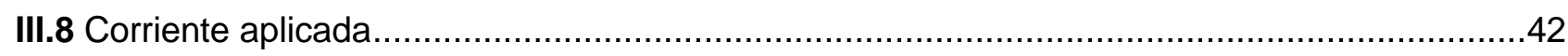

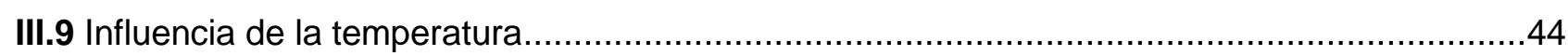

III.10 Influencia de la concentración en la solución acuosa.....................................................45

III.11 Caracterización de Actuadores Bicapa .......................................................................

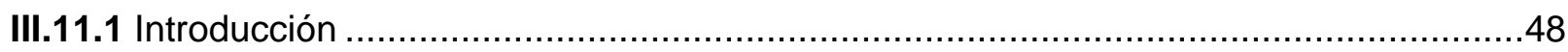

III.11.2 Caracterización del movimiento de los músculos poliméricos tipo bicapa pPy/DBS ...........48

III.11.3 Caracterización del movimiento (velocidad angular/ corriente) del músculo artificial

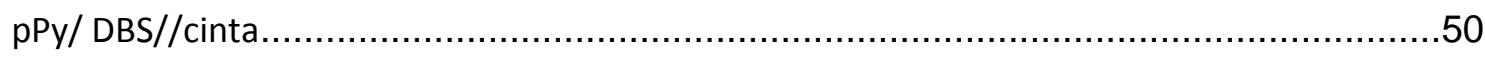

III.11.4 Intercambio de cationes y agua durante la actuación .............................................52

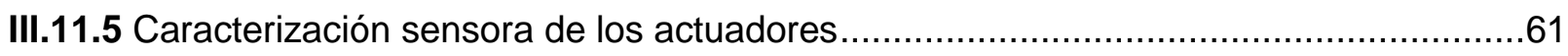

III.11.5.1 Caracterización del actuador como sensor de la densidad de corriente

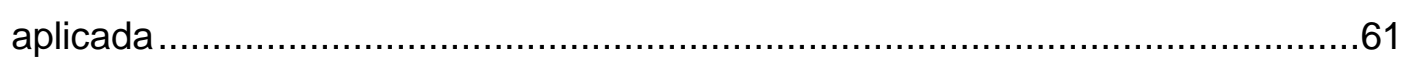

III.11.5.2 Caracterización del actuador como sensor de la temperatura ...........................63

III11.5.3 Caracterización sensora del actuador en función de la concentración del Electrolito ...................................................................65

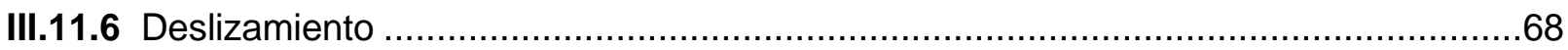

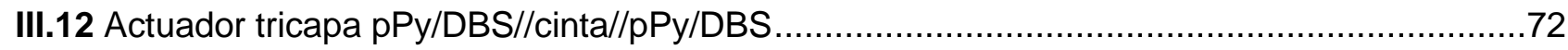

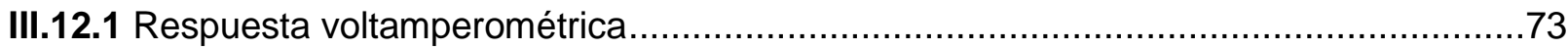

III.12.2 Respuesta dual actuadora/sensora tricapa

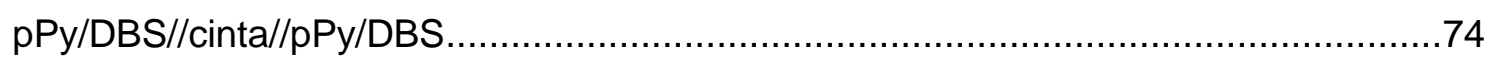

III.12.2.1 Influencia de la corriente eléctrica en el movimiento del 
Actuador tricapa Ppy/DBS//cinta//Ppy/DBS

III.12.2.2 Influencia de la temperatura en el movimiento del

Actuador tricapa Ppy/DBS//cinta//Ppy/DBS .76

III.12.2.3 Influencia de Concentración del electrolito en el movimiento del actuador tricapa $\mathrm{pPy} / \mathrm{DBS} / / \mathrm{cinta} / / \mathrm{pPy} / \mathrm{DBS}$

CONCLUSIONES 80

BIBLIOGRAFÍA. 82 


\section{ÍNDICE DE FIGURAS}

Figura 1.1: Estructura química de polímeros conjugados.

Figura 1.2: Mecanismo de oxidación-reducción de una cadena de polipirrol en presencia de iones perclorato

Figura 1.3: Clasificación de polímeros conductores, (a) Polímeros conductores básicos, (b) Polímeros sustituidos (c) Polímeros autodopados (d) Co-polímeros,(e). mezclas poliméricas, (f) materiales híbridos $(\mathrm{g})$ composites. 6

Figura 1.4: Voltamperograma de un polímero conductor ................................................. 8

Figura 1.5: Expansión y contracción de volumen en polipirrol, de un polímero con intercambio de cationes

Figura 1.6: Dispositivos conductores basados en Polímeros Conductores (PC) de combado

Figura 1.7: Actuador electroquimiomecánico de desplazamiento angular tipo bicapa

Figura 1.8: Diagrama representativo de un actuador tricapa. Las flechas rosas y rojas indican el gradiente generado en la interfase pPy/cinta no-conductora

Figura 2.1: Celda electroquímica de polimerización, se observan los 2 contraelectrodos, el electrodo de trabajo y el electrodo de referencia.

Figura 2.2: Técnica de separación de películas de pPy del sustrato de polimerización.

Figura 2.3: Celda electroquímica para la caracterización de películas de pPy.

Figura 2.4: a) Densidad de corriente aplicada durante experimentos cronopotenciométricos

y b) respuesta del potencial a la corriente aplicada durante la oxidación y reducción de la película (cronopotenciograma)

Figura 2.5: Película autosoportada de pPy

Figura 2.6: Registro voltamperomémetrico de control de una película pPy antes (inicial) y y después (final) de una serie de experimentos.

Figura 2.7: Secuencia de armado de un actuador tipo bicapa película//cinta adhesiva................28

Figura 2.8: Conectividad de un actuador polimérico tricapa.....................................................29

Figura 2.9: Sistema 3D-Grab

Figura 3.1: Voltamperograma cíclico obtenido de una película pPy/DBS autosoportada a $6 \mathrm{mV} / \mathrm{s}$ entre diferentes límites catódicos $(-0.4,-0.6,-0.8,-1,-1.2,-1.4)$ y un único límite anódico $0.6 \mathrm{~V}$ respecto al electrodo de referencia $\mathrm{Ag} / \mathrm{AgCl}$ en disolución acuosa $0.1 \mathrm{M} \mathrm{LiClO} 4$. a temperatura ambiente .

Figura 3.2: Voltamperograma cíclico obtenido de una película pPy/DBS autosoportada a $6 \mathrm{mV} / \mathrm{s}$ en disolución acuosa de $0.1 \mathrm{M} \mathrm{LiClO} 4$ a temperatura ambiente 
Figura 3.3: Voltamperogramas cíclicos entre +0.55 y $-1.3 \mathrm{~V}$ respecto al electrodo de referencia $\mathrm{Ag} / \mathrm{AgCl}$ obtenidos de una película pPy/DBS autosoportada a diferentes velocidades de barrido de $50 \mathrm{mV} / \mathrm{s}$ a $1 \mathrm{mV} / \mathrm{s}$ en disolución acuosa 0.1 M LiClO4 a temperatura ambiente

Figura 3.4: Raíz cuadrada de la velocidad de barrido de una película pPy/DBS en $\mathrm{mV} / \mathrm{s}$ frente al máximo medido de corriente en mA. Valores obtenidos de la Figura 3.3 ......33

Figura 3.5: Voltamperograma cíclico obtenido de una película autosoportada pPy/DBS a $4 \mathrm{mV} / \mathrm{s}$ en una disolución acuosa $0.1 \mathrm{M} \mathrm{LiClO} 4$ a temperatura ambiente, en los intervalos de potenciales $-2.5 \mathrm{a}+2.5$ (primer y segundo ciclado) y de $-3 \mathrm{a}+3 \mathrm{~V}$ (tercer ciclado). La línea continua, indica el primer ciclo la línea punteada en rojo el segundo ciclo y la línea punteada en azul el tercer ciclo

Figura 3.6: Respuestas (a) Voltamperométricas y (b) culovoltamétricas de una película pPy/DBS autosuportada hasta diferentes potenciales catódicos $(-1,-1.5$ and $-2 \mathrm{~V})$ en disolución acuosa $0.1 \mathrm{M}$ de $\mathrm{LiClO} 4$. A una velocidad de barrido $6 \mathrm{mV} \mathrm{s}-1$

Figura 3.7: Respuesta culovoltamétrica obtenida de una película autosoportada pPy/DBS en disolución acuosa $0.1 \mathrm{M}$ de $\mathrm{NaCl}$ a velocidad de barrido de $6 \mathrm{mV} / \mathrm{s}$, a temperatura ambiente. Muestra las variaciones abruptas de pendiente relacionadas con las variaciones bruscas en la velocidad de reacción $(\mathrm{dQ} / \mathrm{dE}=$ $1 /$ Fmd [A - ] / dE ). Dichos cambios son debidos a las cuatro transiciones estructurales dentro de la película, manejados por las reacción y descrito por el modelo ESCR: oxidación-reducción, oxidación-compactación, reducciónrelajación y reducción-hinchazón. Además la película polimérica presenta una nueva variación estructural lenta en el estado reducido- hinchado que puede ser debido a una organización micelar o lamelar del DBS y agua

Figura 3.8: Voltamperogramas obtenidos de una película autosoportada pPy/DBS entre $1.3 \mathrm{~V}$ y $0.65 \mathrm{~V}$ a una velocidad de $6 \mathrm{mV} / \mathrm{s}$ en disolución acuosa $0.1 \mathrm{M} \mathrm{LiClO} 4 \mathrm{a}$ temperatura ambiente durante diferentes ciclos (se muestran los ciclos $2,10,20,30,40$ y 50$)$

Figura 3.9: Relación de la carga eléctrica específica consumida durante los voltamperogramas mostrados en la figura 3.8 con el número de ciclo.

Figura 3.10: Registros cronoamperométricos obtenidos de una película pPy/DBS en una disolución acuosa $0.1 \mathrm{M} \mathrm{LiClO} 4$ obtenidos aplicando $0.4 \mathrm{~V}$ tras una prepolarización a un potencial catódico de $-1.2 \mathrm{~V}$ durante diferentes tiempos.

Figura 3.11: Representación doble logarítmica del tiempo a la máxima corriente (tmax) obtenido en la figura 3.10 y el tiempo de prepolarización

Figura 3.12: Carga de oxidación (Qoxid) determinada de los registros cronoamperométricos de película pPy/DBS (Figura 3.10) en función del tiempo de prepolarización 
$\mathrm{t}(\mathrm{c}) \mathrm{a}-1.2 \mathrm{~V}$

Figura 3.13: Cronopotenciogramas normalizados (mismo potencial de inicio) obtenidos al someter la película de pPy/DBS a ondas cuadradas consecutivas de corrientes, desde $\pm 1.5 \mathrm{a} \pm 24 \mathrm{~mA}$, durante el tiempo requerido en cada caso para consumir una carga constante de $180 \mathrm{mC}$, en solución acuosa $0.1 \mathrm{M}$ de LiClO4 y temperatura ambiente

Figure 3.14: Corriente eléctrica consumida de una película de pPy/DBS a) anódica, b) catódica durante los cronopotenciogramas mostrados en la Figura 3.13

Figura 3.15: Cronopotenciogramas normalizados (mismo potencial inicial) obtenidos de una película autosoportada pPy/DBS a diferentes temperaturas cuando: a) $0.8 \mathrm{~mA} \mathrm{y}$ b)-0.8mA se aplican a la película 60s en disolución acuosa $0.1 \mathrm{M} \mathrm{LiClO4} \mathrm{a}$ temperatura ambiente

Figura 3.16: Energía eléctrica específica consumida de película pPy/DBS a diferentes temperaturas, obtenida por integración de los cronopotenciogramas mostrados en la Figura 3.15

Figura. 3.17: Cronopotenciogramas obtenidos de una película autosoportada pPy/DBS en disoluciones acuosas $\mathrm{LiClO} 4$ con diferentes concentraciones de electrolito $(0.09,0.15,0.25,0.30,0.40,0.50 \mathrm{M})$ aplicando: (a) $+2.25 \mathrm{~mA}$ y (b) $-2.25 \mathrm{~mA}$ durante 40 s a temperatura ambiente

Figura 3.18: Variación de la energía eléctrica consumida durante los cronopotenciogramas mostrados en la figura 3.17 en función de la concentración del electrolito

Figura 3.19: Movimientos de combamiento de un actuador pPy/DBS//cinta: a, a') flujo de corriente anódica $\left.b, b^{\prime}\right)$ Representación de la distribución de iones en el músculo c, c') flujo de corriente catódica

Figura 3.20 a): Relación lineal entre la corriente aplicada y la velocidad angular obtenida para un movimiento de \pm 30 o de un músculo pPy/DBS//cinta en una disolución acuosa $0.1 \mathrm{M} \mathrm{LiClO4}$ a temperatura ambiente aplicando diferentes corrientes eléctricas b) carga eléctrica consumida por el músculo para realizar los movimientos descritos para (a).

Figura 3.21: Voltamperogramas de control obtenidos en disolución acuosa $0.1 \mathrm{M}$ de $\mathrm{NaCl}$ entre $-1 \mathrm{~V}$ y $0.2 \mathrm{mV}$, a $6 \mathrm{mV} / \mathrm{s}$ a temperatura ambiente con un desplazamiento del músculo de $50^{\circ}$ después de: (a) experimentos galvanostáticas en cloruro de rubidio $(\mathrm{RbCl})$ y $(b)$ experimentos galvanostáticas en sal de cloruro de magnesio $\mathrm{MgCl} 2$

Figura 3.22: Respuestas cronopotenciométricas obtenidas al aplicar diferentes corrientes 
constantes (indicadas en la Figura) de una bicapa pPy/DBS//cinta en diferentes electrolitos acuosos $0.1 \mathrm{M}$ de las diferentes sales indicadas en la Figura a 25 C. (a) las respuestas anódicos en LaCl3. (a ') respuestas catódica en LaCl3; (b) las respuestas anódicos en $\mathrm{CaCl}$;. (b ') respuestas catódica en $\mathrm{CaCl2,} \mathrm{(c)}$ respuesta anódica de $\mathrm{RbCl}$; (c ') la respuesta catódica de $\mathrm{RbCl}$

Figura 3.23: Evolución de los ángulos descritos por una bicapa pPy/DBS//cinta en función de la carga específica consumida bajo el flujo de diferentes corrientes constantes (de $\pm 0.4 \mathrm{a} \pm 3 \mathrm{~mA}$ ) para diferentes tiempos (se obtuvieron resultados para 11 diferentes corrientes aplicadas), en disoluciones acuosas $0.1 \mathrm{M}$ de las sales estudiadas

Figura 3.24): Relación entre cada pendiente de la Figura 3.23 y el volumen del concomitante catión intercambiado (b) Las relaciones entre cada pendiente de la Figura 3.23 y el volumen aparente solvatado (catión intercambiado más las moléculas de agua calculada)

Figura 3.25: Cronopontenciogramas obtenidos a un músculo bicapa pPy/DBS//cinta caracterizado: a) anódicas y b) catódicas aplicando diferentes corrientes durante el tiempo necesario para obtener desplazamientos de $30^{\circ}$ en una disolución acuosa $0.1 \mathrm{M} \mathrm{LiClO} 4$ a temperatura ambiente.

Figura 3.26: Evolución de la energía eléctrica consumida durante un desplazamiento de $30^{\circ}$ para diferentes densidades de corriente aplicadas, de un actuador pPy/DBS//cinta moviéndose en una disolución acuosa0.1M LiClO4 a temperatura ambiente

Figura 3.27: Cronopotenciogramas de evolución del potencial a diferentes temperaturas para un actuador pPy/DBS//cinta moviéndose durante 30 en una disolución acuosa 0.1M LiClO4 aplicando una corriente de $\pm 1.5 \mathrm{~mA}$.

Figura 3.28: Evolución de la energía eléctrica consumida por un actuador pPy/DBS//cinta realizando en un movimiento de $30^{\circ}$ obtenida por integración de los cronopotenciogramas mostrados en la figura 3.27 en función de la temperatura a la que realizó el movimiento.

Figura 3.29: Cronopotenciogramas obtenidos durante el desplazamiento de un actuador pPy/DBS//cinta aplicando una corriente de $\pm 1 \mathrm{~mA}$ en una disolución acuosa con diferentes concentraciones de $\mathrm{LiClO} 4$ :a temperatura ambiente a) anódicos y b) catódicos.

Figura 3.30: Variación del consumo de energía específica de un actuador pPy/DBS//cinta en respuesta al desplazamiento de $30^{\circ}$ en soluciones acuosas de $\mathrm{LiClO} 4$ obtenidos de la figura 3.29 
Figura 3.31. (a): Respuesta voltamperométrica (V/i) de un actuador pPy/DBS como

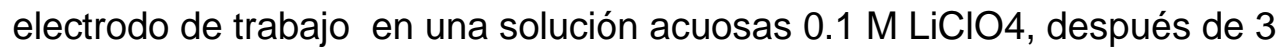
ciclos de estabilización consecutivos, cuando se somete a ciclos potenciales de $-2,5 \mathrm{~V}$ a $1 \mathrm{~V}$, de $-1.2 \mathrm{~V}$ a $0.6 \mathrm{~V}$ o de $-1 \mathrm{~V}$ a $0.4 \mathrm{~V}$, a una velocidad de barrido de $(6 \mathrm{mV} / \mathrm{s})$. (b) respuestas Culovoltamétrica $(\mathrm{Q} / \mathrm{V})$ obtenida por integración de los voltamperogramas de (a), (c). Las fotos de c1 a c10 muestran la posición de flexión del músculo correspondiente a los puntos del 1 al 10. (d) Evolución de la carga consumida, de la respuesta culovoltamétrica y evolución paralela del ángulo descrito por el músculo.

Figura 3.32: Voltamperograma registrado de un actuador tricapa pPy/DBS//cinta//pPy/DBS a una velocidad de barrido de $5 \mathrm{mV} / \mathrm{s}$ entre los límites potenciales de $-0.8 \mathrm{~V}$ y $0.3 \mathrm{~V}$ utilizando disolución acuosa $0.1 \mathrm{M} \mathrm{LiClO4}$ como electrolito a temperatura ambiente.

Figura 3.33: Movimientos angular de una tricapa pPy/DBS//cinta//pPy/DBS (amplitud, \pm 60 grados) en disolución .

Figura 3.34: Respuesta cronopotenciométrica anódica (a) y catódica (b) de una tricapa pPy/DBS//cinta//pPy/DBS aplicando diferentes corrientes en una disolución acuosa $0.1 \mathrm{M} \mathrm{LiClO} 4$ a temperatura ambiente durante el tiempo necesario para obtener movimientos angulares de $\pm 45^{\circ}$

Figura 3.35: Energía eléctrica consumida por gramo de pPy/DBS en función de la corriente eléctrica aplicada obtenida por integración de los cronopotenciogramas de la Figura 3.34. R2 es el coeficiente de correlación del ajuste lineal. a es la sensibilidad de los sensores anódicos (subíndice a) y catódicos (subíndice c); $\theta$ es el ángulo desplazado.

Figura 3.36: Respuesta anódica (a) y catódica (b) cronopotenciométrica de una tricapa $\mathrm{pPy} / \mathrm{DBS} / /$ cinta//pPy/DBS moviéndose al aplicar una onda cuadrada de corriente de $\pm 3 \mathrm{~mA}$ durante $20 \mathrm{~s}$ para obtener movimientos angulares de $\pm 45^{\circ}$ en una disolución acuosa $0.1 \mathrm{M} \mathrm{LiClO4a} \mathrm{diferentes} \mathrm{temperaturas}$

Figura 3.37: Energía eléctrica específica consumida por una tricapa a diferentes temperaturas durante los experimentos de la Figura 3.35 (a) y 3.35 (b). R2 es el coeficiente de correlación del ajuste lineal; a es la sensibilidad de los sensores anódicos (subíndice a) y catódicos (subíndice c); $\theta$ es el ángulo desplazado

Figura 3.38: Respuesta cronopotenciométrica anódica (a) y catódica (b) de una tricapa $\mathrm{pPy} / \mathrm{DBS} / / \mathrm{cinta} / / \mathrm{pPy} / \mathrm{DBS}$ obtenida aplicando un flujo de corriente de $\pm 3 \mathrm{~mA}$ para obtener movimientos angulares $\pm 45^{\circ}$ en diferentes concentraciones de una disolución acuosa de $\mathrm{LiClO} 4$.a temperatura ambiente. 
Figura 3.39: Variación de las energías eléctricas específicas (obtenido por la integración de las respuestas actuales correspondientes a las Figuras 3.37 a y 10 b) con el logaritmo de la concentración experimental del electrolito. R2 es el coeficiente de correlación del ajuste lineal. a es la sensibilidad de los sensores anódicos (subíndice a) y catódicos (subíndice c); $\theta$ es el ángulo desplazado 


\section{ÍNDICE DE TABLAS}

Tabla I.1: Conductividad eléctrica de algunos polímeros conductores dopados con

diferentes moléculas

Tabla I.2: Propiedades electroquímicas de polímeros conductores relacionadas a funciones y órganos biológicos

Tabla III.1: Cargas específicas de oxidación y reducción de los voltamperogramas de control realizados para los diferentes músculos en el electrolito de referencia (disolución acuosa $0.1 \mathrm{M} \mathrm{NaCl}$ ) entre -1 y $0.2 \mathrm{~V}$ frente a $\mathrm{Ag} / \mathrm{AgCl}$ a $6 \mathrm{mV} / \mathrm{s}$, antes (precontrol) y después (post-control) de los experimentos en los diferentes electrolitos.

Tabla III.2: Oxidación y reducción de los picos obtenidos a partir de las respuestas precontrol y post-control voltamperométricas de las bicapas $\mathrm{pPy} / \mathrm{DBS} / / \mathrm{cinta}$ en 0.1 $\mathrm{M}$ disolución acuosa de $\mathrm{NaCl}$ entre -1 y $0.2 \mathrm{~V}$ frente a $\mathrm{Ag} / \mathrm{AgCl}$ en $6 \mathrm{mV} / \mathrm{s}$

Tabla III.3: Radios de cationes, volumen cristalográfico de los cationes y la masa activa de las películas pPy/DBS inmersas en cada electrolito estudiado. Los ángulos descritos por la bicapa pPy/DBS//cinta como una función de la carga específica consumida (por miligramo) por el flujo de las diferentes corrientes constantes (de 0.4 a $3 \mathrm{~mA}$ ) para diferentes tiempos (para una carga específica constante $40 \mathrm{mC} / \mathrm{mg}$ ) a través de los músculos artificiales en soluciones acuosas $0.1 \mathrm{M}$ de los electrolitos estudiados.

Tabla III4: Magnitudes relativas (en soluciones acuosas $0,1 \mathrm{M}$ ) de las sales estudiadas relacionadas con los: ángulos descritos por el músculo de $\mathrm{CsCl}$ (Figura 4) para una carga específica constante de $40 \mathrm{mC} / \mathrm{mg}$; radio cristalográfico y los volúmenes cristalográficos, en disolución acuosa $\mathrm{CsCl}$ (considerado como el sistema de referencia).

Tabla III.5: Número de moléculas de agua intercambiadas, por cationes intercambiados o por electrones extraídos o inyectados, durante la actuación del músculo (reacción), asumiendo de que en $0.1 \mathrm{M} \mathrm{CsCl}$ se intercambian sólo los cationes; en la composición final de las películas de polipirrol reducidas 


\section{GLOSARIO DE TERMINOLOGÍA}

\begin{tabular}{|c|c|}
\hline $\mathrm{ACN}$ & Acetonitrilo \\
\hline AFM & Microscopía de fuerza atómica \\
\hline BS & Benceno-sulfonato \\
\hline BL & Barrido lineal \\
\hline C & Coulombio \\
\hline CA & Cronoamperometría \\
\hline CB & Banda de conducción \\
\hline $\mathrm{ClO}_{4}^{-}$ & Ion de perclorato \\
\hline CE & Contraelectrodo \\
\hline $\mathbf{C P}$ & Cronopotenciometría \\
\hline CV & Voltametría cíclica \\
\hline DBS & Dodecilbencenosulfonato \\
\hline DBSA & Acido dodecilbencensulfónico \\
\hline DRX & Difracción de Rayos X \\
\hline $\mathrm{E}_{\mathrm{ac}}$ & Energía de activación \\
\hline$E_{d}$ & Energía \\
\hline EAPs & Polímeros electroactivos \\
\hline$E_{g}$ & Brecha de energía \\
\hline$E_{1}$ & Potencial inicial \\
\hline$E_{2}$ & Potencial final \\
\hline$E_{\text {máx }}$ & Potencial máximo alcanzado en una CV \\
\hline$E_{p a}$ & Potencial de máximo anódico u oxidativo \\
\hline$E_{p c}$ & Potencial de máximo catódico o reductor \\
\hline ESCR & Estimulación electroquímica de la relajación conformacional \\
\hline EQCM & Microbalanza electroquímica de cristal de cuarzo. \\
\hline eV & Electronvoltio \\
\hline FTIR & Infrarrojo por Transformada de Fourier \\
\hline HR & Húmedad Relativa \\
\hline ICPs & Polímeros Intrinsecamente Conductores \\
\hline$I_{\mathrm{pa}}$ & Corriente de máximo anódico \\
\hline$I_{p c}$ & Corriente de máximo catódico \\
\hline $\mathbf{i}_{\mathbf{f}}$ & Densidad de corriente \\
\hline$I_{1}$ & Corriente inicial \\
\hline $\mathbf{I}_{2}$ & Corriente final \\
\hline $\mathrm{LiClO}_{4}$ & Perclorato de Lítio \\
\hline
\end{tabular}




$\begin{array}{ll}\text { Mpa } & \text { Mega Pascales } \\ \mathbf{m C} & \text { milicoulombio } \\ \mathbf{m V} & \text { milivolts } \\ \mathbf{m g} & \text { miligramo } \\ \mathbf{N a D B S} & \text { Sodio Dodecilbencenosulfonato } \\ \mathbf{P C} & \text { Polímeros Conductores } \\ \mathbf{P y} & \text { Pirrol (monómero) } \\ \mathbf{p P y} & \text { Polipirrol } \\ \mathbf{p P y} / \mathbf{D B S} & \text { Película de polipirrol, sintetizada en solución } 0.2 \text { Py/ 0.2M DBSA } \\ \mathbf{p P y} / \mathbf{L i C O}_{4} & \text { Película de polipirrol, sintetizada en solución } 0.1 \text { Py/ 0.1M LiClO } 4 \\ \mathbf{p P y} / \mathbf{D B S} / / \mathbf{p P y} / \text { LiCO } \\ \mathbf{p P y} / \mathbf{m i x t o}_{\text {H20 }}\end{array}$




\section{Capítulo I}

\section{Introducción}

Hoy en día existe un gran interés por el diseño de sistemas robóticos artificiales inspirados en sistemas biológicos. ${ }^{1}$ El desarrollo de dichos sistemas se centra en todos los aspectos de los mismos, desde la búsqueda de nuevos materiales que imiten el funcionamiento de los materiales de los que están compuestos los seres vivos y simplifique el diseño de los robots, hasta el desarrollo de los componentes individuales, o la totalidad, de los robots imitando el comportamiento de los seres vivos.

Esta tesis presenta la caracterización de polímeros conductores de base polipirrol con intercambio de cationes durante sus reacciones electroquímicas, su caracterización electroquímica y su aplicación a actuadores electro-quimio-mecánicos de bicapa y tricapa (músculos artificiales). El propósito final sería poder llegar a diseñar motores poliméricos a partir de materiales blandos, húmedos, con contenido iónico que imiten el comportamiento de los músculos naturales de los seres vivos. Los músculos naturales son los actuadores más importantes en los sistemas biológicos. Existe un creciente esfuerzo en investigación para el diseño de nuevos materiales blandos que imiten el comportamiento de los actuadores biológicos con altas potencialidades para ser aplicados como dispositivos actuadores/sensores en el desarrollo de nuevos robots zoomórficos o antropomórficos.

Fijándonos en los músculos de los seres vivos un impulso nervioso llega al músculo desde el cerebro a través del sistema nervioso y libera una corriente iónica (calcio) en el sarcómero. La variación de concentración de calcio dispara una reacción química (hidrólisis de ATP) y dicha reacción produce cambios conformacionales del sistema actina-miosina generando la contracción muscular y el movimiento órgano correspondiente. Cuando un polímero conductor está en presencia de un electrolito (disolvente con una sal disuelta), el paso de una corriente eléctrica produce una reacción electroquímica con intercambio de iones y disolvente desde la disolución y el correspondiente cambio de volumen del polímero. Se ha propuesto a los polímeros conductores como el modelo más simple de materiales (geles densos y reactivos) del interior de una célula. ${ }^{2}$ Los eventos consecutivos que originan el cambio de volumen de los polímeros conductores imitan, uno por uno, los que tienen lugar en los músculos naturales. Es por ello que a los actuadores basados en polímeros conductores se les ha denominado músculos artificiales, actuadores poliméricos o motores poliméricos. ${ }^{3}$ 
Una de las propiedades de las reacciones químicas es que su velocidad de reacción y la energía de la reacción es una función de las condiciones energéticas (térmicas, químicas, mecánicas, eléctricas, ópticas, etc.) en las que actúa: la reacción siente las condiciones ambientales. De forma similar, un músculo natural es capaz de sentir elementos de su entorno como su estado de fatiga, la fuerza que necesita realizar para desplazar un objeto, si es capaz de desplazarlo o no, la temperatura de trabajo, etc. La propiedad por la cual el cerebro es capaz de conocer en todo momento la posición, velocidad de movimiento, dirección del movimiento, presencia de obstáculos, resistencia de esos obstáculos al movimiento producido por los músculos mientras se mueven se conoce con el nombre de propiocepción. ${ }^{4,5}$ Los músculos artificiales también sienten las condiciones en las que trabajan tales como la presencia de objetos en su camino, las condiciones químicas, térmicas o eléctricas: son músculos-sensores. Los músculos artificiales sirven para desarrollar dispositivos con capacidades propioceptivas $^{6-8}$.

\section{I.1 Polímeros conductores}

Los polímeros conductores son materiales poliméricos que, como los metales, pueden ser oxidados y reducidos y en estado oxidado conducen la corriente eléctrica. Entre dichos materiales destacan los polímeros basados en anillos de pirrol, furano y tiofeno, ya que poseen una buena estabilidad ambiental además de buenas propiedades eléctricas y ópticas ${ }^{6,9}$.

Politiofeno




\section{Capítulo I}

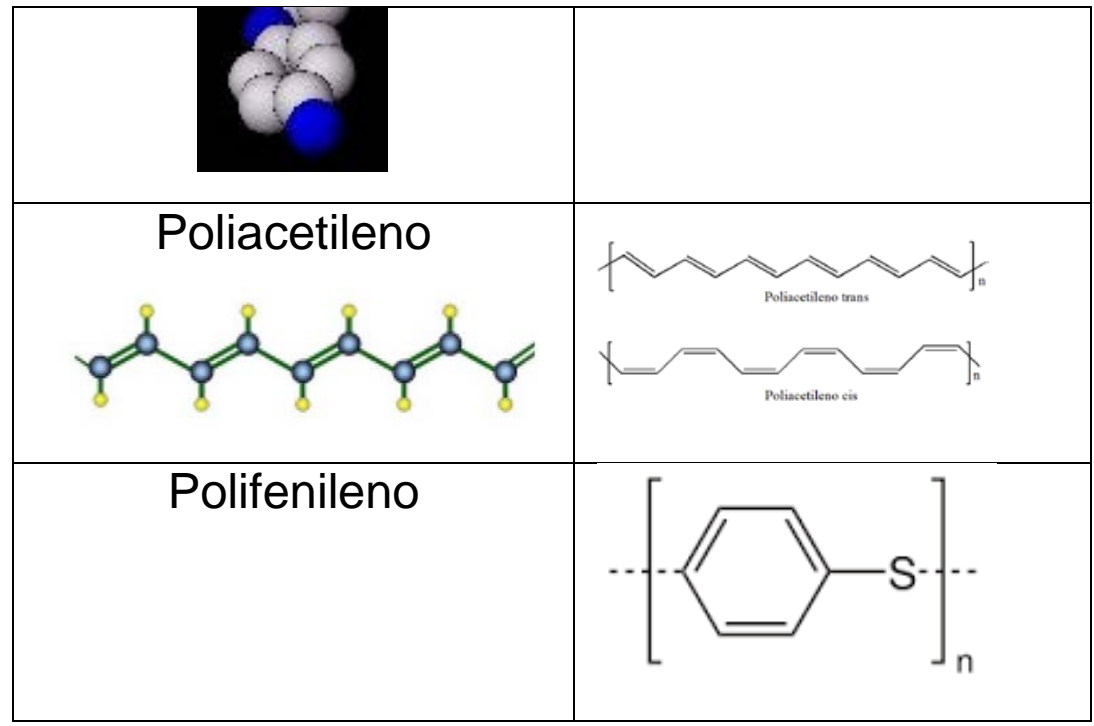

Figura 1.1 Estructura química de algunos polímeros conjugados.

Los polímeros conductores, de los que se pueden observar ejemplos en la Figura 1.1 tienen, en estado neutro, una estructura de dobles enlaces alternada de enlaces sencillos (sigma, fijos) y dobles enlaces $(\pi) .{ }^{10,11}$ La presencia de enlaces sigma entre cada dos unidades monoméricas hace que el polímero sea aislante y las unidades monoméricas puedan rotar libremente alcanzando múltiples conformaciones. Al oxidarse cambia la estructura electrónica de la cadena polimérica generándose dobles enlaces conjugados en forma de radical-cationes (polarones en la terminología física): enlaces conjugados planos entre varias unidades monoméricas que soportan el flujo de electrones, aumentando la conductividad cuanto mayor es la concentración de esas estructuras conjugadas en el polímero. La presencia de cargas positivas en las cadenas poliméricas requiere la entrada de contraiónes desde la disolución para balancear la carga y mantener la electroneutralidad. Los movimientos conformacionales que se producen en las cadenas para permitir la entrada de contraiones, las cargas positivas y los contraiónes hacen que se genere espacio libre entre las cadenas poliméricas y fuerzan la entrada de disolvente para equilibrar la presión osmótica entre dentro y fuera del polímero. Las cadenas poliméricas reactivas, los contraiones y el disolvente (generalmente agua) constituyen un gel denso que imita, en su contenido más simple, la matriz intracelular de las células vivas ${ }^{12}$. En la Figura 1.2 se ilustra, como ejemplo y de manera breve, el mecanismo de oxidación/reducción de una cadena de polipirrol en presencia de perclorato de litio. Durante la oxidación, los electrones son extraídos de las cadenas de polipirrol, generando un exceso de cargas positivas en las cadenas y la deslocalización de los enlaces doble-simple. El exceso de cargas positivas es compensado por contraiónes 
de la disolución para mantener la electroneutralidad del material. Durante la reducción ocurre lo contrario, los electrones son inyectados en las cadenas poliméricas, expulsando los contraiónes y volviendo a su conjugación original (enlace simple-doble enlace).

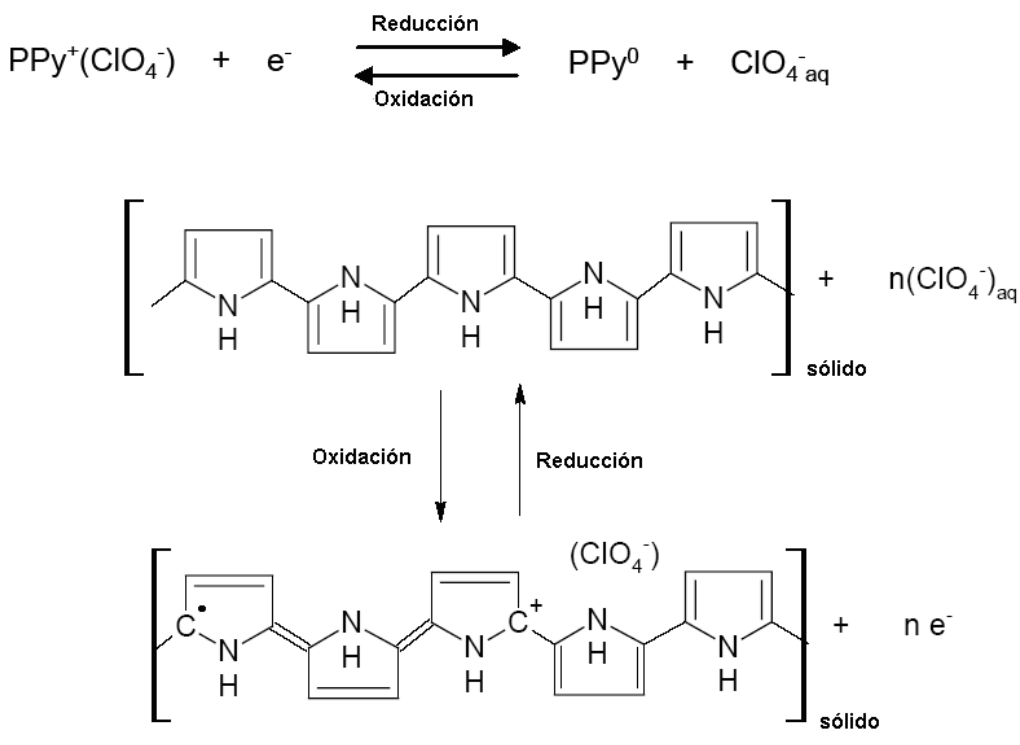

Figura 1.2 Mecanismo de oxidación-reducción de una cadena de polipirrol en presencia de iones perclorato.

El cambio de composición del material debido a la inclusión de los contraiónes en el polímero conductor para mantener la electroneutralidad también se conoce con el nombre de dopado por sus similitudes con el dopado de semiconductores inorgánicos. Como en ellos hay polímeros que se pueden oxidar, dopado de tipo $p$, y otros que se pueden reducir inyectando electrones a la cadena (dopado de tipo $n$ ) con entrada de cationes para compensar las cargas. Durante el dopado del polímero se produce el cambio en la distribución de los dobles enlaces originando el movimiento de las cadenas poliméricas con el cambio en las posiciones relativas de los átomos (conformaciones) que permiten la generación de volumen libre y la introducción de los contraiónes.

Con la variación en la composición (polímero/contraión) del material durante la reacción, se producen cambios en las propiedades eléctricas, mecánicas, ópticas, químicas, térmicas, etc. del material de varios órdenes de magnitud. La conductividad y la conjugación, van aumentando con la oxidación, pudiendo calcularse variaciones teóricas de la conductividad de una cadena aislada en un intervalo entre $10 \times 10^{3}$ hasta $50 \mathrm{~S} / \mathrm{cm}$, como se muestra en la tabla $\mathrm{I} .1^{13}$. El nivel del dopado puede ser reversiblemente controlado mediante la reacción electroquímica empleando técnicas 


\section{Capítulo I}

electroquímicas sencillas. La variación de las propiedades de los polímeros conductores dependerá de la técnica y condiciones de síntesis del polímero, el agente dopante y las características del medio en que se polimeriza (tipo de sustrato, temperatura, disolvente, luminosidad, etc. ${ }^{14-17}$, así como su estado de dopado (grado de oxidación-reducción) del material.

El descubrimiento de estos materiales mereció la concesión del premio Nobel de Química del año 2000 a los investigadores A. Heeger, A. McDiarmid y H. Shirakawa por su contribución al descubrimiento y desarrollo de polímeros orgánicos conductores. ${ }^{7,18-23}$.

Tabla I.1 Conductividad eléctrica de polímeros conductores dopados con diferentes moléculas.

\begin{tabular}{|c|c|c|}
\hline Polímero & Material Dopante & $\begin{array}{l}\text { Conductividad } \\
\text { aproximada } \\
(\mathrm{S} / \mathrm{cm})\end{array}$ \\
\hline Poliacetileno & $\mathrm{I}, \mathrm{Br}, \mathrm{Na}, \mathrm{AsF}$ & 10000 \\
\hline Polipirrol & $\mathrm{BF}-, \mathrm{ClO}_{4^{-}}$, tosilato & $500-7500$ \\
\hline Politiofeno & $\begin{array}{c}\mathrm{BF}-, \mathrm{ClO}_{4^{-}}, \text {tosilato, } \\
\mathrm{FeCl}-\end{array}$ & 1000 \\
\hline Poli(3-alquitiofeno) & $\mathrm{BF}-, \mathrm{ClO}_{4^{-}}, \mathrm{FeCl}-$ & $1000-10000$ \\
\hline Polifenil-sulfuro & AsF & 500 \\
\hline Polifenilenvinileno & AsF & 10000 \\
\hline Politienilenovinileno & AsF & 2700 \\
\hline Polifenileno & AsF,Li,K & 1000 \\
\hline Polisotianafteno & BF-, $\mathrm{ClO}_{4^{-}}$ & 50 \\
\hline Polifurano & $\mathrm{BF}-, \mathrm{ClO}_{4}^{-}$ & 100 \\
\hline Polianilina & $\mathrm{HCl}$ & 200 \\
\hline
\end{tabular}

\section{I.2 Clasificación de polímeros conductores}

Cada uno de los monómeros con cada uno de los posibles agentes dopantes e influidos por las condiciones experimentales de síntesis generan polímeros conductores por polimerización. Podemos clasificar a los polímeros conductores en diferentes familias que se enumeran a continuación (figura 1.3) ${ }^{24}$ : 


\section{Capítulo I}

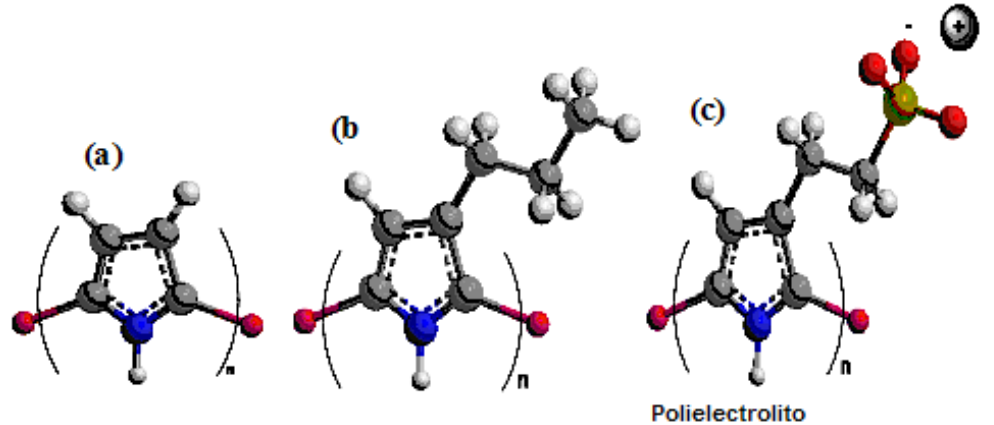

(e) )

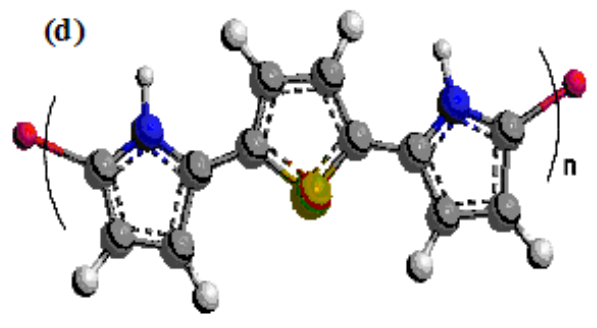

(f)
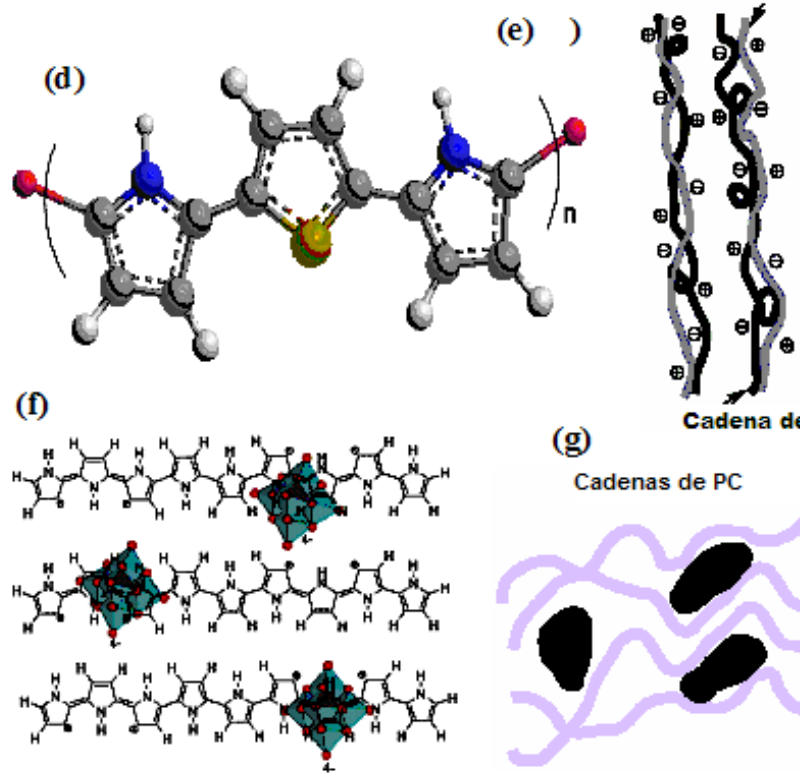

(g)

Cadena de polímero

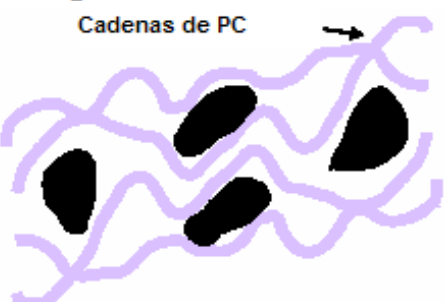

Figura 1.3 Clasificación de polímeros conductores, (a) Polímeros conductores básicos, (b) Polímeros sustituidos (c) Polímeros autodopados (d) Co-polímeros, (e) mezclas poliméricas, (f) materiales híbridos $(g)$ composites.

a. Polímeros conductores básicos: los más representativos son polipirrol ${ }^{25-3} 1$, polianilina ${ }^{32-37}$, politiofeno ${ }^{38,39}$, polifurano ${ }^{40-42}$ y sus derivados.

b. Polímeros sustituidos ${ }^{43-46}$ : Uno o varios átomos de hidrógeno, en el monómero base es sustituido por otro grupo químico. Se puede utilizar diferentes grupos para que los polímeros sustituidos tengan diferentes propiedades eléctricas 0 mecánicas.

c. Polímeros autodopados ${ }^{40,47-49}$ : los sustituyentes poseen un grupo iónico que compensa la carga inyectada en la cadena durante la reacción electroquímica.

d. Co-polímeros ${ }^{50-54}$ : dos o más unidades monoméricas están presentes en la cadena polimérica.

e. Aleaciones polímero/macro-ion El material sintetizado oxidado contiene macroiones que no se intercambian en los procesos redox; en esta familia se pueden encontrar los siguientes grupos:

f. Materiales híbridos ${ }^{55-57}$ : el macro-ion añadido es inorgánico 
g. Aleaciones poliméricas ${ }^{58-61}$ : el macro-ion es un polielectrolito o un macro-ion orgánico.

h. Composites ${ }^{62-66}$ : formados con diferentes materiales orgánicos o inorgánicos.

De las familias de polímeros conductores solo algunos de los posibles materiales han sido sintetizados y estudiados. La inmensa mayoría de los posibles materiales están aún por sintetizar.

\section{I.3 Síntesis de Polímeros conductores}

Existen diferentes técnicas para la síntesis de polímeros conductores: oxidación electroquímica, oxidación química, plasma, polimerizaciones foto-iniciadas, etc. ${ }^{67,68}$. En la síntesis del polímero conductor, las condiciones específicas del proceso determinan la estructura molecular del polímero obtenido, afectando sus propiedades químicas, mecánicas y eléctricas ${ }^{24,60,69-75}$.

\section{I.4 Electropolimerización del pirrol}

De las técnicas de síntesis mencionadas, la polimerización electroquímica es la más utilizada dado que se puede controlar la morfología y el espesor de la película, resultando en polímeros más limpios comparados con los sintetizados químicamente ${ }^{7}$, 76-82. La electropolimerización se produce por oxidación del monómero que se encuentra disuelto en un disolvente, que también contiene una sal disuelta mediante la aplicación de una corriente electrónica a través de un sustrato conductor (electrodo). Se generan radicales cationes que por condensación originan oligómeros cada vez más largos. Los oligómeros se oxidan para generar sales de los mismos (cadenas con cargas positivas y aniones para balancear la carga). De este modo, la síntesis y el dopado son procesos que se dan simultáneamente. El anión se incorpora en el polímero para asegurar una neutralidad eléctrica de la película.

\section{I.5 Oxidación y reducción de Polímeros Conductores}

Una vez sintetizados los polímeros conductores, ya sea formando una película sobre el metal en el que se generaron, o cómo electrodos autosoportados formados por las películas gruesas una vez despegadas del electrodo, se pueden usar como electrodos en una celda electroquímica y allí oxidarlos y reducirlos (dopar/desdopar) reversiblemente. Para ello se usan como electrodo de trabajo en un electrolito, en el que se pueden someter a técnicas electroquímicas tales como barridos cíclicos de potencial (voltamperometría cíclica), saltos consecutivos de potencial 
(cronoamperometría) o a ondas cuadradas de corriente (cronopotenciometría). En la figura 1.4 se muestra la respuesta voltamperométrica del polímero cuando se sometió a un barrido cíclico de potencial. Al avanzar el potencial eléctrico hacia valores más anódicos (la derecha en la figura) comienza a fluir una corriente positiva hasta que se alcanza un máximo debido a que los electrones se arrancan de las cadenas poliméricas: el material se oxida. Al retroceder el potencial la corriente se hace catódica (negativa) debido a la inyección de electrones a las cadenas poliméricas para anular las cargas positivas y el material se reduce.

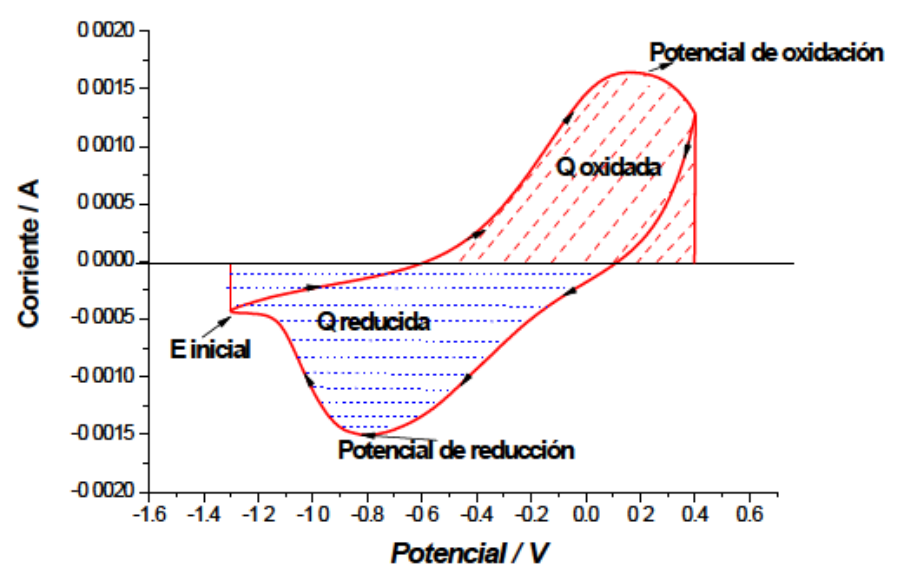

Figura 1.4 Voltamperograma de un polímero conductor.

Los polímeros conductores caracterizados electroquímicamente en electrolitos líquidos, se convierten en materiales blandos, húmedos y de composición variable (polímero, disolvente y contraiónes), debido a la entrada de contra-iones para mantener la electroneutralidad y disolvente para mantener la presión osmótica, tal y como se mencionó en el apartado I.1.

Dependiendo de la familia de polímeros conductores hay dos mecanismos básicos de reacción (dopado):

a) Intercambio aniónico. Los polarones positivos generados en la cadena se compensan con aniones que entran desde la disolución durante la oxidación. Este mecanismo lo siguen las familias $a, b, f, y ~ g{ }^{83,84}$ de la figura 1.3. La reacción simplificada, eligiendo una película de polipirrol, será:

$$
\begin{aligned}
& \left(p P y^{0}\right)_{s}+n\left(A^{-}\right)_{a q}+m(\text { Disolvente }) \underset{\text { Reducción }}{\stackrel{\text { Oxidación }}{\text { Cadena oxidada }}}\left[\left(p P y^{n+}\right)_{s}\left(A^{-}\right)_{n}(\text { Disolvente })_{m}\right]_{\text {gel }}+n\left(e^{-}\right)_{\text {metal }} \\
& \text { Cadena neutra }
\end{aligned}
$$


Donde pPy representa a las cadenas de polipirrol, $\mathrm{A}^{-}$, son los aniones presentes en la disolución y que entran en la estructura polimérica durante la reacción para mantener la electroneutralidad del polímero, Disolvente son moléculas de disolvente que se intercambian con la disolución para mantener el equilibrio osmótico en el interior de la membrana polimérica, formando un gel denso (indicado por el subíndice gel); y e representan los electrones extraídos/insertados de las cadenas poliméricas durante la oxidación/reducción.

b) Intercambio catiónico. Este mecanismo se presenta para las familias c y e de la figura 1.3. Se da cuando el polímero se electrogeneró en presencia de un macro-ion dando lugar a una aleación macro-ion-polímero conductor. Al ser oxidado en presencia de un electrolito el macro-ion permanece atrapado en el interior del material debido a sus dimensiones y a las fuertes uniones intermoleculares entre el macro-ion y el polímero ${ }^{85-88}$. Para mantener la electroneutralidad la oxidación/reducción fuerza el intercambio de cationes:

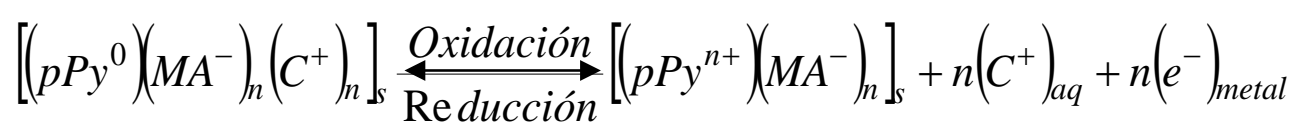

Cadena Neutra

Cadena Oxidada

donde: s significa sólido, $M A^{-}$representa el macro-anión atrapado dentro del polímero durante la polimerización, pPy es la cadena de polipirrol y $\mathrm{C}^{+}$representa un catión, que balancea la carga del macro-anión atrapado.

Las reacción (1.1 y 1.2) producen cargas positivas en la cadena polimérica, siendo este un proceso tipo dopado "p".

En esta tesis se estudiarán y caracterizarán películas de polipirrol generado en presencia de macro-iones orgánicos, así como músculos artificiales basados en estas aleaciones poliméricas con intercambio de cationes siguiendo la reacción (1.2).

\section{I.6 Propiedades electroquímicas de los polímeros conductores.}

Las propiedades de los materiales dependen de su composición. Las reacciones (1.1) y (1.2) provocan el cambio de la composición (polímero/ contraión) del material. El valor de cada una de aquellas propiedades que sean función de la composición se 
podrá modificar dentro de un intervalo mediante la carga invertida en las reacciones: serán propiedades electroquímicas.

De acuerdo con las reacciones (1.1) y (1.2), es posible obtener un gran número de estados intermedio de la reacción (tantos como electrones son extraídos/insertados del material) de forma contínua, reversible y estables. Cada uno de esos estados es un material diferente con una composición diferente, y por lo tanto, propiedades diferentes. A continuación se enuncian las propiedades más importantes que varían con el grado de oxidación/reducción (dopado):

- Propiedades electrocrómicas. Los polímeros conductores sintetizados en forma de películas delgadas son transparentes a la luz, al pasar una corriente eléctrica se produce una reacción de oxidación que deslocaliza los electrones entre varios anillos (polarones). Los nuevos orbitales (enlazantes y antienlazantes) vacíos de electrones tiene distinta energía que los orbitales de partida del polímero neutro, que absorben luz ultravioleta. La reacción genera cromóforos, capaces de absorber la luz visible e infrarroja, cambiando el color. La absorbancia de luz es función de la concentración de polarones en la película (ley de Lambert-Beer) y dicha concentración se puede variar de forma revesible con las reaccines de oxidación/reducción. El cambio controlado de las propiedades electrocrómicas se está utilizando para el desarrollo de ventanas inteligentes, espejos inteligentes, gafas de absorción controlable o pantallas ${ }^{89-93}$.

- Almacenamiento de carga eléctrica. En el interior de la red polimérica, al oxidarse o reducirse, las cadenas poliméricas almacenan cargas eléctricas positivas o negativas, por lo que están siendo usados como electrodos de baterías o para construir baterías totalmente poliméricas ${ }^{94-96}$.

- Electroporosidad. En estado neutro las cadenas del polímero conductor tienen una elevada atracción polímero-polímero, por lo que el material se encuentra compactado con poros intermoleculares de bajo diámetro. Si el polímero conductor se oxida, la estructura se abre y aumenta la distancia entre cadenas, por lo que aumenta el tamaño de los poros. Al aplicar un gradiente de potencial entre ambos lados de la membrana polimérica, los iones que se encuentran cercanos fluyen a través de estos poros $^{90,97-102}$. La conductividad iónica a través de la membrana se puede controlar con el estado de oxidación. También se puede controlar la conductividad relativa de iones con distinto diámetro. 
- $\quad$ Propiedades electroquimiomecánicas. Esta propiedad se debe al cambio de volumen que se da las reacciones 1.1 y 1.2. Para compensar las cargas eléctricas entran en la matriz polimérica contraiónes y agua de la disolución, dando lugar a un aumento de volumen del material. En el proceso inverso las cadenas poliméricas recuperan los electrones expulsando los contraiónes y recuperando su estado inicial, haciendo que el polímero se contraiga. Generalizando, cuando la reaccción supone la entrada mayoriataria de iones y disolvente el volumen del material aumenta, cuando la reacción obliga a la salida de iones y disolvente el volumen del material disminuye. Las reacciones controlan la magnitud y el sentido del cambio de volumen. Los músculos artificiales, son una aplicación de esta propiedad ${ }^{103-106}$ (Figura 1.5).
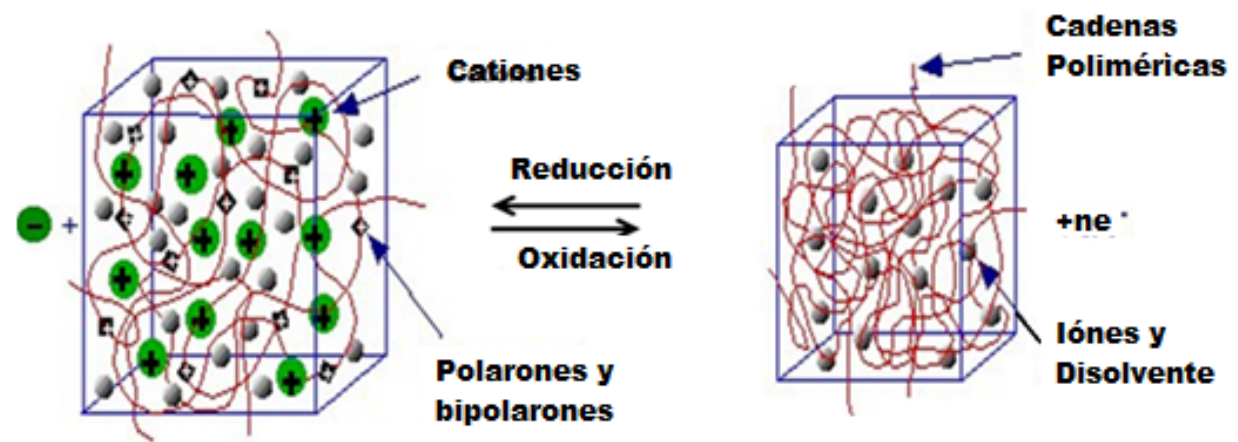

Figura 1.5 Expansión y contracción de volumen en polipirrol, de un polímero con intercambio de cationes.

Los polímeros conductores que tienen un intercambio predominante de cationes (reacción 1.2) durante las reacciones presentan cambios de volumen y movimientos inversos a los de los materiales de intercambio de aniones. El material se expande durante la reducción y se contrae durante la oxidación.

- Propiedades de transducción electrón/ión o electrón/transducción química. En las reacciones 1.1 y 1.3 se tiene una relación entre los electrones inyectados o extraídos del polímero y el número de aniones o cationes intercambiados entre el material y la disolución. Por ello, estas reacciones son adecuadas para el almacenamiento de iones en la red polimérica, así como para su liberación bajo control electroquímico. Esta propiedad los hace candidatos atractivos para aplicarse para la liberación inteligente de fármacos en el cuerpo humano, ${ }^{2}$. 
Muchas de las propiedades mencionadas se corresponden con las que cambian durante la actuación de muchos órganos de los seres vivos. No sólo la composición de los polímeros conductores recuerda la de la matriz intracelular de las células vivas sino que también sus propiedades recuerdan las funciones biológicas desarrolladas por las reacciones en los órganos de los seres vivos. En la tabla 1.2 se muestran las propiedades de los polímeros conductores ligadas a una acción e inspirada en un órgano biológico.

Tabla I.2 Propiedades electroquímicas de polímeros conductores relacionadas a funciones y órganos biológicos ${ }^{107}$

\begin{tabular}{|l|l|l|}
\hline Propiedad & Acción & Órganos \\
\hline Electroquimiomecánica & Cambio de volumen & Músculos \\
\hline Electrocrómica & Cambio de color & Piel mimética \\
\hline Almacenamiento de cargas & Generación de corriente & órganos eléctricos \\
\hline Electroporosidad químico o & Flujo transversal iónico & Membrana \\
\hline $\begin{array}{l}\text { Almacenamiento quicaión química } \\
\text { farmacológico }\end{array}$ & Glándulas \\
\hline Transducción electrón/ión & $\begin{array}{l}\text { Propiedades } \\
\text { Físicas) }\end{array}$ & Bio-sensores \\
\hline Electrón/neurotransmisor & Canal-acción & Interfase nerviosa \\
\hline
\end{tabular}

\section{I.7 Músculos artificiales}

De entre las propiedades de los polímeros conductores modificadas de forma reversible por las reacciones de oxidación/reducción del material una de las más estudiadas es el cambio de volumen. El cambio reversible de volumen imita al que tiene lugar en los músculos naturales durante contracción/relajación. Es por ello que se ha empleado para el desarrollo de músculos artificiales: motores capaces de transformar los pequeños cambios de volumen originados por la reacción a nivel molecular en movimientos macroscópicos y controlables. Los diseños más usuales son bicapas polímero conductor//cinta adhesiva 0 tricapas polímero conductor//cinta//polímero conductor. Los cambios de volumen que la reacción reversible origina en el polímero conductor se transforman en un movimiento macroscópico y reversible de combado (movimiento angular) ${ }^{108-111}$.

Una cadena de un polímero conductor puede ser considerada durante la reacción como un motor molecular electro-quimio-mecánico. La cadena reducida presenta una estructura globular. La cadena pasa por $n$ etapas consecutivas de equilibrio químico o por $n$ estados energéticos conformacionales consecutivos: la estructura se abre para 
permitir la aproximación de los contraiónes a los polarones. La cadena oxidada presenta una estructura extendida (de bastón). El proceso es reversible bajo control electroquímico, siendo $\mathrm{n}$ el número de electrones extraídos/añadidos a cada cadena polimérica durante la oxidación o inyectados durante la reducción. El cambio entre la forma globular y la extendida, bajo control de la reacción electroquímica constituye un motor molecular. ${ }^{112}$

Varias cadenas poliméricas conforman una película de polímero conductor. Las películas que reaccionan con intercambio de aniones (reacción 1.1) se expanden durante la oxidación y se contraen durante la reducción ${ }^{113}$, las de intercambio de cationes (reacción 1.3) presentan cambios de volumen y movimientos opuestos.

Los músculos artificiales basados en polímeros conductores se aproximan a los músculos naturales en varios aspectos fundamentales. Por ejemplo, trabajan a bajo potencial de $100 \mathrm{mV}$ a $2 \mathrm{~V}$ mientras que los músculos naturales funcionan de $60 \mathrm{a}$ $150 \mathrm{mV}$, que es el potencial del pulso nervioso. Los músculos artificiales trabajan tanto en contracción como en expansión, mientras que el musculo natural solamente trabaja en contracción ${ }^{114}$.

El primer actuador basado en polímeros conductores tuvo un tamaño de varios centímetros. Este dispositivo fue patentado por Otero y sus colaboradores en 1992 , en la Universidad del País Vasco, España. Dicho actuador se basó en una bicapa formada por una película de polipirrol dopado con $\mathrm{ClO}_{4}^{-} \mathrm{y}$ otra de un polímero flexible, aislante y adherente $e^{7,115-119}$. Para transformar los movimientos conformacionales moleculares, en movimientos macroscópicos, se diseñó un dispositivo de doble capa polipirrol/película adherente como electrodo en una disolución acuosa. De esta forma, los pequeños cambios de volumen producidos en la interface entre el polímero conductor y la película adherente (de volumen constante) producen un movimiento angular (Figura 1.8). Estos actuadores son capaces de desarrollar un trabajo mecánico y de desplazar un peso, al mismo tiempo que sienten las condiciones del medio.

Algunas desventajas de los actuadores de polímeros conductores incluyen un deslizamiento (creeping) importante en ciclos consecutivos y su tiempo de vida limitado.

l.7.1 Clasificación de los músculos artificiales (actuadores poliméricos
electroquímicos) 
Los actuadores electroquimiomecánicos se pueden clasificar de acuerdo a la trayectoria del I movimiento que producen:

- $\quad$ Lineales.

- $\quad$ De Bending (combado).

Los lineales aprovechan los cambios reversibles de volumen a lo largo de una dimensión del material (longitud) que la reacción electroquímica reversible produce en el material.

Los tipos más comunes de actuadores lineales son: fibras y láminas ${ }^{120-126}$, tubos y láminas con soporte metálico ${ }^{127-133}$ y combinación de dispositivos angulares ${ }^{131-133}$. En la caracterización de este tipo de dispositivos la relación que se utiliza para el desplazamiento longitudinal, es el porcentaje de desplazamiento longitudinal (diferencia de longitud final, $L$, del dispositivos respecto a su longitud inicial $L_{0}$ )

Los de combado (bicapa o tricapa) mediante la inclusión de una capa soporte adhesiva, que mantienen constante su volumen durante el flujo de corriente, transforman los pequeños movimientos longitudinales en el polímero conductor en el combado reversible del dispositivo originado movimientos angulares macroscópicos del extremo libre.

La figura 1.6 presenta los diferentes dispositivos actuadores basados en polímeros conductores ( $A$, B son actuadores bicapa y C, D son del tipo tricapa) que contienen una o más películas de polímero conductor y una capa soporte. La capa soporte puede ser un polímero aislante, un polímero metalizado, un hidrogel, un metal o un polielectrolito sólido.

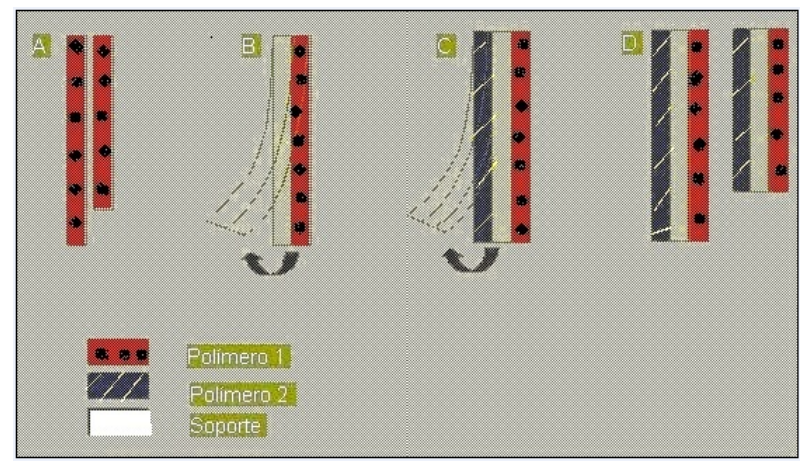

Figura 1.6. Dispositivos conductores basados en Polímeros Conductores (PC) de combado.

Un actuador electroquimiomecánico de bicapa se ilustra en la figura 1.7. El polímero conductor se adhiere a un polímero no-conductor. Durante el dopado, el polímero 


\section{Capítulo I}

conductor se expande empujando al polímero no conductor y de esta forma el actuador exhibe un desplazamiento angular. Durante el desdopado el polímero conductor se encoge y el dispositivo recupera la posición original.

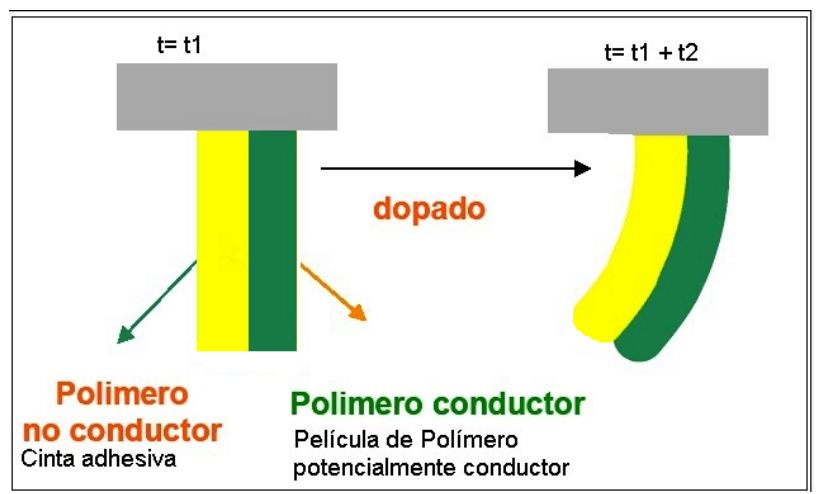

Figura 1.7 Actuador electroquimiomecánico de desplazamiento angular tipo bicapa.

El comportamiento de actuador tricapa se ilustra en la figura.1.8, al aplicar una corriente continua una de las películas de polímero conductor se dopa y expande y empuja al dispositivo, la otra se desdopa y se contrae y tira del dispositivo ${ }^{142-145}$. En la figura 1.8a la película de pPy del lado derecho actúa como ánodo, se oxida y se expande por entrada de aniones y agua y del lado izquierdo como cátodo, se reduce y se contrae con expulsión. La oxidación de la película y su expansión, debido a la entrada de los aniones necesarios para neutralizar las cargas positivas, genera un gradiente de tensiones de expansión en la interfase pPy/cinta no-conductora. De manera simultánea, la reducción de la película del lado izquierdo provoca la salida de contraiónes del polímero y su consecuente contracción con la formación de un gradiente de tensiones de contracción en su interfase con la cinta no-conductora (Figura 1.8b). El gradiente de tensiones provoca un combado del dispositivo hacia el lado derecho (figura 1.8a), y estos gradientes de tensiones son de signo contrario. Al estar las dos películas de pPy enfrente una de la otra, los gradientes de tensiones se complementan cuando se mueven, sumando sus efectos ${ }^{138-143}$.

El movimiento inverso se presenta cuando la película de la izquierda actúa como cátodo y la de la derecha como ánodo. La tricapa siempre se combará hacia el lado que se encuentra la película de pPy que actúa como cátodo y se contrae ${ }^{115,144-153}$. 


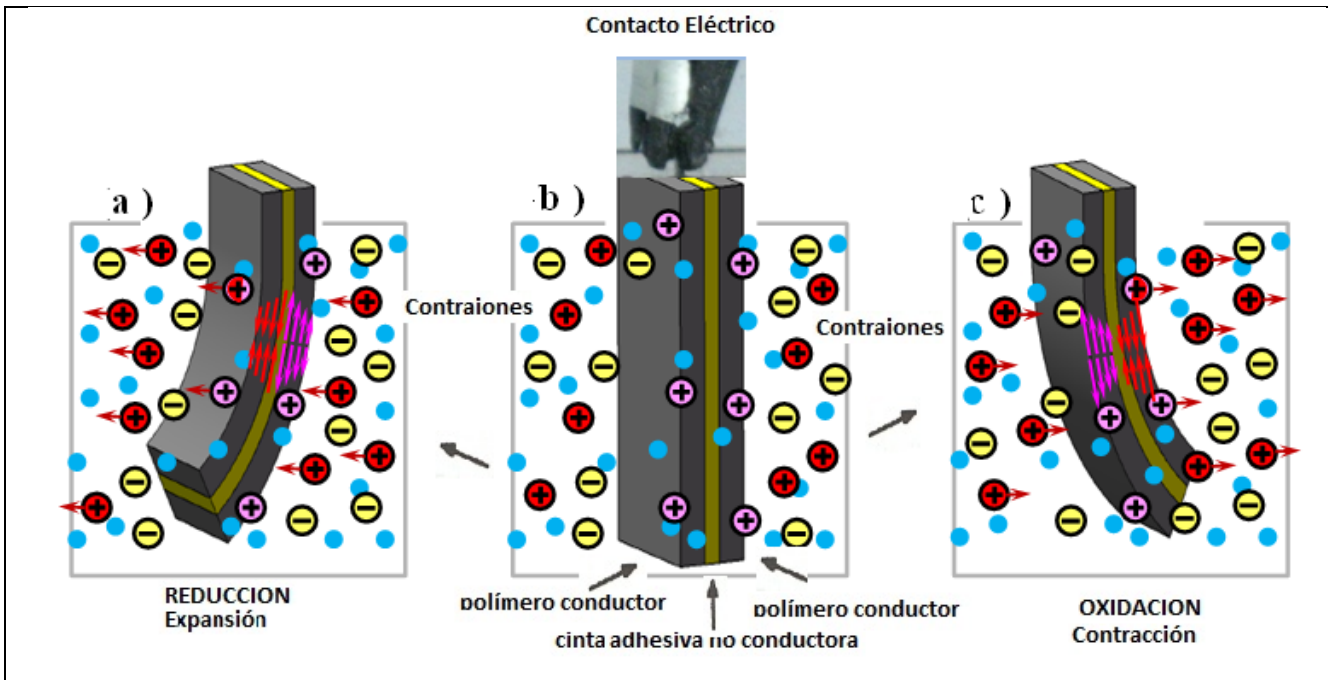

Figura 1.8 Diagrama representativo de un actuador tricapa. Las flechas rosas y rojas indican el gradiente generado en la interfase pPy/cinta no-conductora ${ }^{7,154-156}$.

La oxidación de una de las películas, al pasar una corriente entre las dos, implica la reducción de la otra en la misma magnitud: por las dos pasa la misma carga. El actuador tipo tricapa permite procesos anódicos y catódicos simultáneos, siendo más compacto ya que el electrodo y el contra-electrodo están empaquetados en un mismo dispositivo, evitándose reacciones electroquímicas no deseadas en la interfase disolución-contra-electrodo metálico y la pérdida de energía eléctrica consumida para producir las reacciones de descarga del agua del contra-electrodo metálico requerido para el funcionamiento de las bicapas. Ambas reacciones son empleadas en desarrollar trabajo mecánico ${ }^{53-56,157-160}$.

Al estar el movimiento originado por las reacciones 1.1 y 1.2 la carga eléctrica (el número de electrones) definirá el estado de oxidación del material y, por lo tanto la posición de músculo artificial. La carga eléctrica por unidad de tiempo (la corriente) definirá la velocidad de reacción, por lo tanto la velocidad de movimiento del músculo y el sentido de flujo de la corriente (anódica o catódica) define el sentido de movimiento del músculo (el de las agujas del reloj, o el contrario, para los músculos que se comban dando movimientos circular de su extremo libre). Los músculos artificiales son motores poliméricos farádicos (controlados por las leyes de Faraday) ${ }^{161}$.

Las variaciones de volumen relacionadas con el grado de oxidación del polímero, estarán controladas también con la carga consumida para alcanzar ese estado de oxidación. De esta forma, debe de existir una relación biunívoca entre cargas y volúmenes $\mathrm{y}$, por lo tanto entre la carga y la posición del extremo libre de la 
bicapa. Al dispositivo correspondiente se le denomina electroquimioposicionador ${ }^{162}$.

\section{I.8 Propiedades actuadoras/sensoras de los Polímeros Conductores}

Durante las reacciones 1.1 y 1.2 las propiedades anteriormente mencionadas y mostradas en la tabla 1.2 pueden variar simultáneamente. De esta forma, sería posible obtener dispositivos multifuncionales utilizando películas de polímero conductor, en las que varias propiedades varían a la vez impulsadas por la misma reacción del material. Fijándonos simultáneamente en la variación de volumen y en la del potencial eléctrico del material durante la reacción se están desarrollando músculos artificiales que sienten por sí mismos las condiciones experimentales (mecánicas, químicas, térmicas, eléctricas) de trabajo.

Los cambios en la composición intermedia del material están ligados a un equilibrio electroquímico entre especies poliméricas, contraiónes y moléculas de disolvente dentro del polímero, iones y moléculas de disolvente en la solución, y electrones expulsados del interior de la cadena desde el electrodo de trabajo (metal). Cualquier variable que afecte a la velocidad de la reacción durante la actuación muscular producirá un cambio de la energía necesaria para que la reacción y el movimiento muscular continúen. Si el músculo funciona impulsado por una corriente constante el efecto de cualquier cambio de variable se notará, inmediatamente, en la evolución del potencial muscular mientras se mueve. El músculo será un sensor de las condiciones en que está trabajando, como hacen los músculos naturales (músculos hápticos) ${ }^{163}$. Esta propiocepción artificial ha sido demostrada para músculos artificiales basados en polímeros conductores con intercambio de aniones, es decir, siguiendo la reacción $(1.1)^{164}$.

\section{I.9 Potencialidades de los Polímeros Conductores.}

Los polímeros conductores son materiales con capacidades y propiedades comunes a los plásticos siendo muy flexibles y de bajo coste, lo que los posiciona como excelentes candidatos para ser aplicados en diferentes sectores como el de la medicina (área de farmacología y biomedicina) ${ }^{165-169}$ y diversos sectores industriales ya que pueden ser utilizados en aplicaciones tecnológicas como el desarrollo energético, baterías ${ }^{170}$, fotodiodos y Leds ${ }^{171}$ debido a su capacidad para ser semiconductores y conductores de electricidad, con niveles de operación muy bajos (capaces de competir con la tecnología actual basada en 
semiconductores como el $\mathrm{Si}$ ). Las variaciones simultáneas en sus propiedades actuadoras y sensoras durante una reacción electroquímica fácilmente controlable, ofrecen la posibilidad de desarrollar dispositivos multifuncionales que se pueden aplicar en diferentes áreas del conocimiento, tales como robótica, dónde permitirían desarrollar robots más simples, baratos y fiables.

A día de hoy se han sintetizado y caracterizado materiales orgánicos y biocompatibles que pueden ser capaces de intercambiar aniones y/o cationes en un medio o responder eléctricamente a un cambio de concentración. ${ }^{172}$ Combinando materiales de intercambio de cationes y materiales con intercambio de aniones, se abre la posibilidad de trabajar en el diseño y construcción de interfaces entre el sistema nervioso ${ }^{173,174}$. Estos materiales son capaces de transformar las señales eléctricas que producen los ordenadores y no entienden las neuronas en señales iónicas y químicas, que si entienden las neuronas a través de los canales iónicos de las dendritas, construyendo traductores, o transductores de las señales electrónicas en señales iónicas, y viceversa. Se abre una posibilidad real para la comunicación entre equipos electrónicos y el cerebro (conexión de cámaras de video como ojos artificiales) o entre el cerebro y equipos electrónicos (manejo de brazos robóticos con las señales directas del cerebro).

Por otro lado el desarrollo de los músculos artificiales permite por ejemplo el trabajo en aplicaciones de micro robótica en equipos quirúrgicos, en catéteres para facilitar el manejo de equipos ópticos como posicionadores y como sensores-actuadores en sistemas de detección ${ }^{175}$.

Cada día existe mayor interés por parte de la Agencia Espacial Estadounidense (NASA) y de la Agencia Espacial Europea (ESA) en la aplicación de actuadores poliméricos. Ya existen varias empresas que producen y han producido dispositivos basados en polímeros conductores: Quantum Technology Pty. Ltd, Micromuscles $\mathrm{AB}^{176}$ (Compañía cerrada recientemente), Molecular Mechanics, empresa asociada al Centro Tecnológico de Massachussets del MIT, USA, Artificial muscles INC (AMI ${ }^{177}$, Danfos Polypower A/S compañía danesa dedicada al giro de plásticos inteligentes ${ }^{178}$, EMPA ${ }^{179}$, Environment Robots Incorporated, Nanosonic Inc ${ }^{180,181}$. Un ejemplo del creciente interés en estos materiales y aplicaciones es la reciente adquisición de "Artificial Muscles" por parte de la multinacional Bayer Material Science. 
Actualmente una de sus limitaciones más importantes para ser utilizados en masa es la falta de modelos teóricos electroquímicos y mecánicos, que expliquen sus propiedades reactivas, así como los procesos elementales que ocurren en estos materiales, por lo que la investigación experimental de sus aspectos fundamentales y el desarrollo de modelos teóricos supondría un gran avance para el desarrollo de nuevos productos industriales basados en estos materiales biomiméticos.

Algunos de estos aspectos fundamentales son abordados en esta tesis para un material que ha demostrado tener una excelentes propiedades mecánicas, pero que todavía se ignoran aspectos fundamentales: el polipirrol dopado con dodecilbencenosulfonato (pPy/DBS). El fin último, al que no se alcanza en esta tesis, sería la utilización de dicho material como actuador para desarrollos en robótica.

La presente tesis consta de tres capítulos, en este Capítulo I se ha ofrecido una introducción a los conceptos más importantes sobre el estado del arte en Polímeros Conductores, el Capítulo II presenta la metodología experimental utilizada en esta tesis para la obtención de los resultados y el Capítulo III presenta los resultados obtenidos para la caracterización electroquímica del polímero conductor de intercambio catiónico pPy/DBS, así como la caracterización de los actuadores electro-quimio-mecánicos de doble y triple capa con la discusión de los resultados alcanzados, finalmente se presentan las Conclusiones a esta tesis y las referencias bibliográficas. 


\section{METODOLOGÍA EXPERIMENTAL.}

En este capítulo se describen los procedimientos y técnicas utilizados para sintetizar y caracterizar las películas poliméricas de base polipirrol (pPy) con Ácido dodecilbenceno sulfónico (DBSH) como agente dopante, así como se enuncian los dispositivos, equipos, materiales y reactivos utilizados. También se describen los procedimientos para la construcción de los músculos artificiales de bicapa o de tricapa y la caracterización de su movimiento angular bajo diferentes técnicas electroquímicas.

\section{II.1 Materiales y reactivos}

En la preparación de las películas poliméricas de pirrol se utilizaron los siguientes reactivos:

a) Pirrol, Py (Sigma, 98\%), purificado por destilación en condiciones de vacío y almacenándolo a $-10^{\circ} \mathrm{C}$ hasta su uso.

b) Ácido dodecilbenceno sulfónico, DBSH (Sigma, 70wt\% disolución en 2propanol), usado directamente tal y como se recibió.

c) Perclorato de litio, $\mathrm{LiClO}_{4}$ (Aldrich, $95 \%$, ACS reactivo analítico), usado directamente tal y como se recibió.

d) Cloruro de sodio, $\mathrm{NaCl}$ (Sigma-Aldrich), usado como se recibió.

e) Cloruro de magnesio, $\mathrm{MgCl}_{2}$ (Sigma-Aldrich), usado como se recibió.

f) Cloruro de calcio, $\mathrm{CaCl}_{2}$ (Sigma-Aldrich), usado como se recibió.

g) Cloruro de potasio, KCl (Sigma-Aldrich), usado como se recibió.

h) Cloruro de rubidio, $\mathrm{RbCl}$ (Sigma-Aldrich), usado como se recibió.

i) Cloruro de cesio, $\mathrm{CsCl}$ (Sigma-Aldrich), usado como se recibió.

j) Cloruro de lantano (III) heptahidratado, $\mathrm{LaCl}_{3} 7 \mathrm{H}_{2} \mathrm{O}$ (Sigma-Aldrich), usado como se recibió.

k) Placas de acero inoxidable S316.

I) Agua ultrapura destilada obtenida de un equipo de Millipore Milli-Q.

\section{Métodos.}

\section{II.2.1 Preparación del sustrato de polimerización.}

Como electrodos de trabajo se utilizaron láminas de acero inoxidable S316 de dimensiones $1.8 \mathrm{~cm} \times 5 \mathrm{~cm}$, y contraelectrodos de $3 \mathrm{~cm} \times 5.5 \mathrm{~cm}$. Para su limpieza las láminas fueron lijadas utilizando lijas del número P400 (water proof CB5), terminando con un pulido en una pulidora mecánica marca Imptech Europe Modelo Grinder polisher 1PM-10V. Posteriormente la lámina se lavó y se enjuagó nuevamente 
con agua destilada, se colocaron en el interior de baño de ultrasonidos durante quince minutos y posteriormente se sumergieron en acetona antes de su utilización.

\section{II.2.2 Purificación del monómero}

El monómero fue destilado en condiciones de vacío utilizando una bomba de vacío (modelo MZ 2C marca SCHOTT) a 7 mbar de presión. La destilación se realizó entre $38^{\circ} \mathrm{C}$ y $42^{\circ} \mathrm{C}$. El monómero destilado fue recolectado en un recipiente y almacenado en el congelador a una temperatura de entre -8 y $-10^{\circ} \mathrm{C}$ hasta su utilización.

\section{II.2.3 Electrogeneración de películas poliméricas de base pirrol}

La síntesis del polímero se realizó utilizando un potenciostato-galvanostato Autolab PGSTAT100. La electropolimerización se efectuó en una celda electroquímica de tres electrodos y de compartimiento simple. El medio electrolítico utilizado fue $50 \mathrm{ml}$ de disolución acuosa conteniendo pirrol destilado en una concentración $0.2 \mathrm{M}$, y DBSH, también en una concentración 0.2M.

El electrodo de trabajo, después de ser limpiado tal y como se ha descrito en la sección II.2.1, se colocó en la parte media de la celda electroquímica, quedando dentro de la disolución un área de $5 \mathrm{~cm}^{2}$ por cada uno de los lados. Como contraelectrodos se utilizaron dos electrodos de $3 \mathrm{~cm} \times 5.5 \mathrm{~cm}$ del mismo material, colocados simétricamente a ambos lados del electrodo de trabajo a $1 \mathrm{~cm}$ de distancia con el fin de obtener un campo eléctrico uniforme a ambos lados del electrodo de trabajo. Como electrodo de referencia se utilizó un electrodo marca Metrohm $\mathrm{Ag} / \mathrm{AgCl}$ $3 \mathrm{M} \mathrm{KCl}\left(E=210.5 \mathrm{mV}_{\mathrm{H} / \mathrm{H} 2}\right)$, todos los potenciales en esta tesis están referidos a este electrodo.

La distribución de los electrodos en la celda electroquímica se muestra en la Figura 2.1.

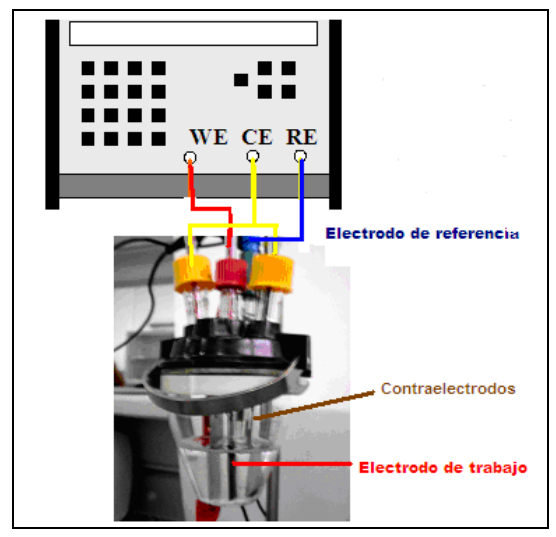

Figura 2.1 Celda electroquímica de polimerización, se observan los 2 contraelectrodos, el electrodo de trabajo y el electrodo de referencia. 
La polimerización se realizó aplicando una densidad de corriente constante $(I)$ de 2 $\mathrm{mA} / \mathrm{cm}^{2}$ durante 2 horas, siendo por tanto la carga total consumida durante la electropolimerización de $72 \mathrm{mC}$. La posible foto-oxidación del monómero fue minimizada realizando el depósito en la oscuridad y con la disolución a una temperatura inicial a $2^{\circ} \mathrm{C}$ continuando hasta llegar a temperatura ambiente. Todas las polimerizaciones se llevaron a cabo con disoluciones recién preparadas. El electrodo de acero, una vez recubierto con poli pirrol, se extrajo de la celda y se sumergió en agua destilada durante 24 horas para eliminar el exceso de DBSH adsorbido en la superficie del polímero.

\section{II.2.4 Separación de las películas de pPy}

Dos películas de polipirrol, una por cada lado del electrodo de trabajo, fueron separadas del acero con un cúter, como se ilustra en la Figura 2.2: se retiró el polímero depositado en los laterales del electrodo y se separaron las dos películas de la parte frontal y posterior del acero. Se obtienen así dos películas autosoportadas de polipirrol/Dodecilbencenosulfonato (pPy/DBS). Dichas películas fueron almacenadas en el refrigerador a una temperatura de entre 5 y $10^{\circ} \mathrm{C}$ hasta su utilización.

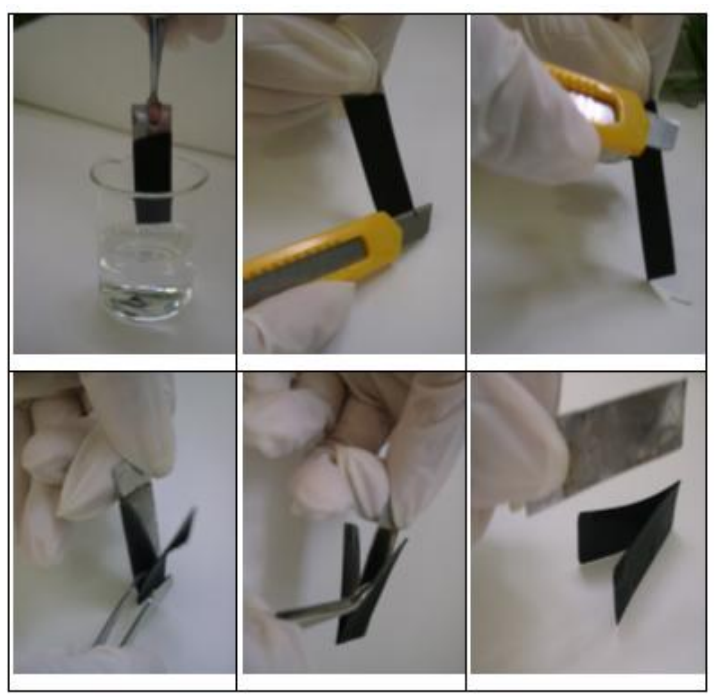

Figura 2.2 Técnica de separación de películas de pPy del sustrato de polimerización

\section{II.2.5. Determinación del espesor}

Para medir el espesor de las películas de pPy/DBS auto-soportadas se usó un micrómetro manual, de marca Comecta Electronic modelo 5900602, con una sensibilidad de $0.1 \mu \mathrm{m}$. Se midió en cada película el espesor en 10 puntos distintos y se utilizó como resultado final el valor promedio para cada película. 


\section{II.3 Revisión de Conceptos básicos:}

Velocidad de barrido: se define como la relación entre la variación lineal de potencial ( $V$, medido frente al electrodo de referencia) que se aplica al electrodo de trabajo con el tiempo (s) durante un barrido lineal de potencial.

$$
\text { Velocidad de barrido }=\square \text { Tiempo(s) (2.1) }
$$

Dónde $\Delta$ indica incrementos.

Carga eléctrica (Q): se define como la integral de la corriente que pasa a través de las películas poliméricas cuando esta está conectada como electrodo de trabajo en el tiempo que dura el movimiento.

$$
Q=\int_{0}^{t}(i \bullet d t)
$$

Donde: $Q$ representa la carga eléctrica (Culombios, $C$ ), i es la corriente eléctrica (Amperios, A), t es el tiempo (segundos, s). La carga específica se expresa como la carga por unidad de masa de material activo $(\mathrm{mC} / \mathrm{mg})$.

Energía eléctrica $\left(E_{\mathrm{e}}\right)$ : se define como la integral del producto del potencial eléctrico por la corriente eléctrica que circula en la película polimérica por el tiempo que pasa por esta:

$$
E_{e} t=\int_{0}^{t} i t E(t) d t
$$

Donde: $i$ es la densidad de corriente específica $\left(\mathrm{mA} \mathrm{mg}^{-1}\right), E$ es el potencial eléctrico $(V)$, t es el tiempo (s) y $E_{e}$ es la energía eléctrica consumida ( $\left.\mathrm{mJ}\right)$.

\section{II.4 Técnicas electroquímicas experimentales e instrumentación}

La caracterización electroquímica del polímero se realizó utilizando un potenciostatogalvanostato marca Autolab PGSTAT100 controlado mediante un ordenador personal con el software General Pourpouse Electrochemical Software (GPES).

La celda electroquímica utilizada para la caracterización se observa en la Figura 2.3.

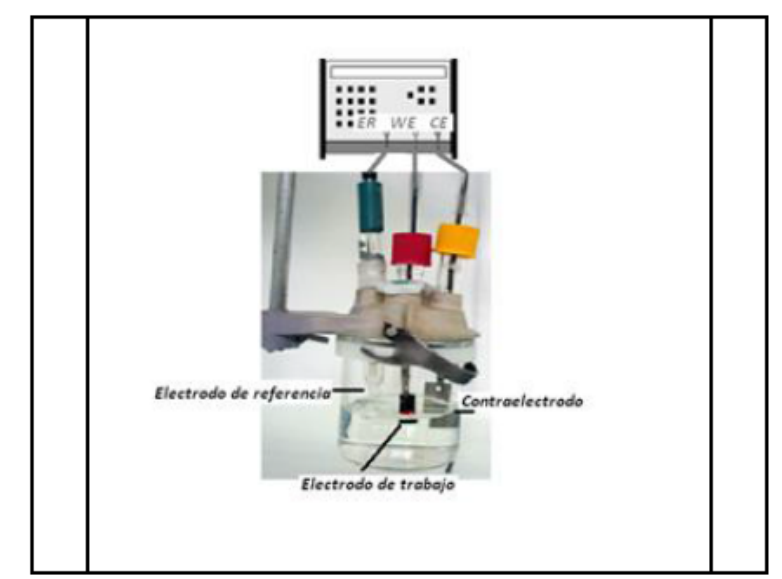

Figura 2.3 Celda electroquímica para la caracterización de películas de pPy 
Las películas, sintetizadas, enjuagadas y pesadas, se colocaron en una disolución acuosa de $\mathrm{LiClO}_{4}$ a temperatura ambiente $\left(25^{\circ} \mathrm{C} \pm 1^{\circ}\right)$ y se caracterizó su respuesta electroquímica mediante diferentes técnicas electroquímicas: Voltamperometría cíclica, cronoamperometría y cronopotenciometría.

\section{II.4.1. Voltamperometría cíclica}

La voltamperometría cíclica es una técnica electroquímica que consiste en realizar un barrido lineal y cíclico de potencial a una velocidad de barrido $(\mathrm{mV} / \mathrm{s})$ contante en una ventana de potencial de trabajo definida por los límites de potencial anódico y catódico entre los que se realiza el barrido. Mientras se aplica dicho barrido de potencial se registra la evolución de corriente que pasa por el sistema. A la representación de dicha corriente medida respecto del potencial aplicado se le denomina respuesta voltamperométrica o voltamperograma ${ }^{182}$.

La densidad de carga eléctrica registrada durante la oxidación o reducción es un parámetro cuantitativo muy utilizado en la caracterización voltamperométrica. Para calcular la carga eléctrica se aplica la ecuación (2.2) teniendo en cuenta la relación existente entre la velocidad de barrido y el tiempo.

Es posible representar dicha carga en función del potencial aplicado, obteniendo así la respuesta culovoltamétrica. Los potenciostatos-galvanostatos empleados dan tanto la respuesta voltamperométrica como la culovoltamétrica de forma automática.

\section{II.4.2 Cronopotenciometría y Cronoamperometría}

La Cronopotenciometría es la técnica electroquímica en la que se aplica entre el electrodo de trabajo y el contraelectrodo el flujo de una densidad de corriente constante mientras se registra la evolución de la diferencia de potencial eléctrico entre el electrodo de trabajo y el electrodo de referencia en función del tiempo transcurrido durante el experimento (respuesta cronopotenciométrica). Es posible combinar diferentes flujos de corrientes constantes aplicando ondas cuadradas de corriente 183,184 .

Esta técnica es empleada con frecuencia para caracterizar el comportamiento electroquímico de los músculos artificiales y su capacidad sensora bajo distintas condiciones experimentales: densidades de corriente, temperatura y concentración de sales $^{185}$.

En la figura 2.4 a), se ilustra, el procedimiento aplicado experimentalmente durante el desarrollo de esta tesis, donde inicialmente se somete al material al flujo de una determinada corriente catódica (en el caso de la figura 2.4 a, - $-0.150 \mathrm{~mA}$ durante $250 \mathrm{~s}$ ) durante un determinado tiempo (reducción del material) para buscar que se estabilice 
a un mismo estado de reducción inicial para cada experimento. Posteriormente se aplican tres ondas cuadradas consecutivas de corriente. Las corrientes y los tiempos que se aplican fueron calculadas con el fin de mantener la cargas de oxidación y reducción constantes (mismos estados redox inicial y final en cada caso). Las respuestas de evolución del potencial del músculo con el tiempo (cronopontenciogramas) se registran tal y como se ilustra en la figura $2.4 \mathrm{~b}$.

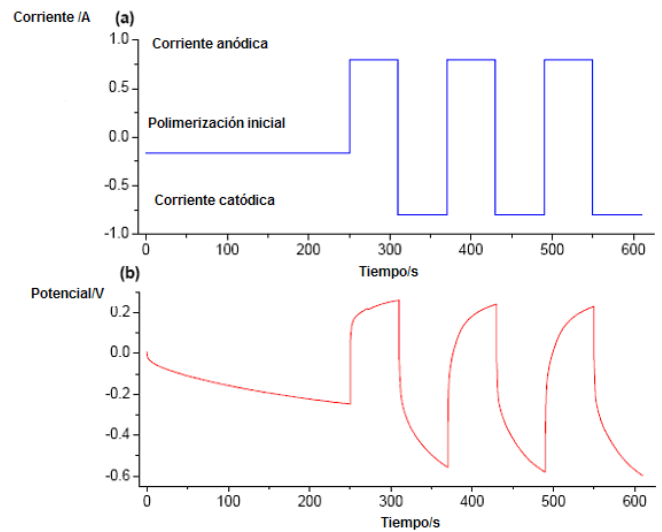

Figura 2.4 a) Densidad de corriente aplicada durante experimentos cronopotenciométricos y b) respuesta del potencial a la corriente aplicada durante la oxidación y reducción de la película (cronopotenciograma)

La cronoamperometría es una técnica electroquímica en la que se aplica al electrodo de trabajo un salto potencial constante, medido con respecto al electrodo de referencia, registrándose la evolución de la corriente eléctrica que fluye entre el electrodo de trabajo y el contraelectrodo para reajustar el potencial electródico (respuesta cronoamperométrica). Esta técnica puede utilizarse realizando un único salto de potencial o realizar varios saltos consecutivos de potencial (lo más habitual en nuestro caso): Se fija el valor inicial y final del potencial catódico/anódico a aplicar, con un determinado tiempo de aplicación de cada uno de estos potenciales. Se aplica al electrodo de trabajo el salto de registrándose la evolución de la corriente que atraviesa el electrodo de trabajo a cada momento ${ }^{183,184}$.

\section{II.5.1 Electrodos poliméricos autosoportados.}

Las películas poliméricas después de haber sido sintetizadas electroquímicamente, enjuagadas con agua destilada y separadas del sustrato metálico, se cortan en tiras del tamaño requerido y se pesan en la balanza marca Sartorius modelo ED2245.

Para evitar el contacto directo entre la pinza metálica y el electrolito por capilaridad se pinta transversalmente una tira de barniz comercial de uñas a ambos lados de la 
película de polímero a una distancia de $1 \mathrm{~cm}$ del borde superior y se deja secar. Con ello se cubren los poros del polímero impidiendo el ascenso del electrolito por capilaridad. Además, dicha tira de pintura delimita la cantidad de material que se meterá en la disolución para su caracterización. Se han reportado en la literatura el estudio de otras películas poliméricas conductoras auto-soportadas ${ }^{185-191}$.

Posteriormente cada una de ellas es suspendida en la celda electrolítica en una posición vertical (Ver Figura 2.5), quedando el nivel del electrolito por debajo del límite inferior de la película de pintura transversal. Esta película autosoportada de pPy/DBS es el electrodo de trabajo polimérico (películas autosoportada), que se conecta en la parte superior con una pinza metálica para garantizar el contacto eléctrico.

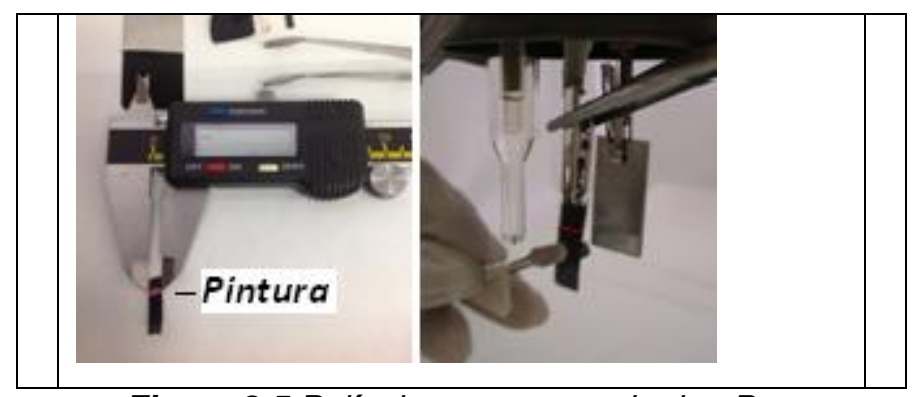

Figura 2.5 Película autosoportada de pPy

En todas las etapas de la experimentación electroquímica, el lado externo que presenta la película durante la electropolimerización se colocó frente al contraelectrodo.

\section{II.5.2 Valoración del estado de las películas o actuadores caracterizados}

En todos los experimentos realizados en esta tesis, tanto de películas autosoportadas, como de músculos de bicapa o tricapa, cuya construcción se describirá más abajo, es necesario realizar un control de su electroactividad. Para ello se someten a un control voltamperométrico, mediante barridos consecutivos de potencial hasta conseguir una respuesta estacionaria. Tanto al inicio como al final de los distintos experimentos se verifica que mantiene su electroactividad mediante otro voltamperograma de control y asegurar así la reproducibilidad de los experimentos.

Un ejemplo de control voltamperométrico puede observarse en la figura 2.6; obtenido de ciclar una película pPy/DBS a $6 \mathrm{mV} / \mathrm{s}$, en un intervalo de 0.4 a $-1.2 \mathrm{~V}$. La diferencia entre los voltamperogramas inicial (antes de los experimentos) y final (tras los experimentos) muestra un pequeño desplazamiento del pico del potencial de oxidación y de reducción hacia la derecha (sin cambios significativos de la carga eléctrica consumida). Este control experimental sirve para evaluar que la película continúa en 
condiciones estables similares a las del experimento inicial, y determina que se pueda continuar con otros experimentos de la misma serie. Cuando la carga consumida durante la oxidación/reducción varía en más de un 10\% respecto al primer control realizado a la película, la película es sustituida por una nueva con una carga específica (por unidad de masa) con los mismos valores que la película inicial.

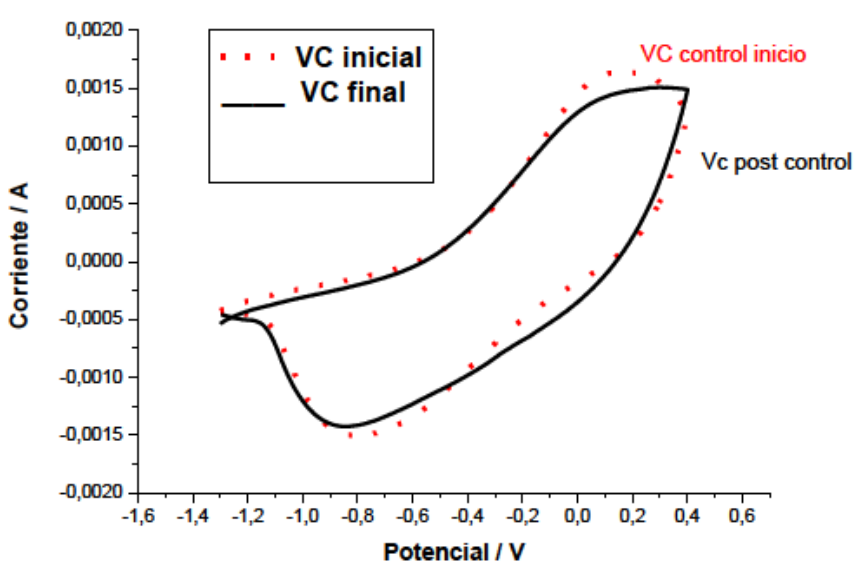

Figura 2.6 Registro voltamperométrico de control de una película pPy antes (inicial) y después (final) de una serie de experimentos.

\section{II.6 Construcción del actuador bicapa: película//cinta adhesiva}

Para construir el actuador bicapa película//cinta adhesiva se siguen los pasos de preparación del material según la sección II.2; posteriormente, para obtener el actuador, se siguen los pasos que se ilustran en la figura 2.7:

a) Se corta la película de pPy a las dimensiones deseadas con un cúter.

b) Se pesa en la microbalanza Sartorius modelo ED2245 y se mide espesor.

c) Posteriormente se coloca sobre una tira de cinta adhesiva doble cara y se pega uniformemente a la superficie de pPy/DBS que estaba en contacto con el metal durante la electropolimerización, teniendo cuidado de que las orillas queden pegadas (ver figura 2.7 paso 1 ).

d) Se corta el excedente de cinta adhesiva (ver figura 2.7 paso 2 ).

e) Se retira la cubierta protectora de la cinta adhesiva (ver figura 2.7 paso 3 ).

f) Se pinta con barniz una tira transversal para evitar la capilaridad y se calcula la proporción de la masa de película debajo de la pintura que pondrá dentro de la disolución para su caracterización.

g) El actuador bicapa se suspende como electrodo de trabajo en la celda electroquímica para su caracterización mediante una pinza metálica para asegurar el contacto eléctrico con el potenciostato (ver figura 2.7 paso 4). 

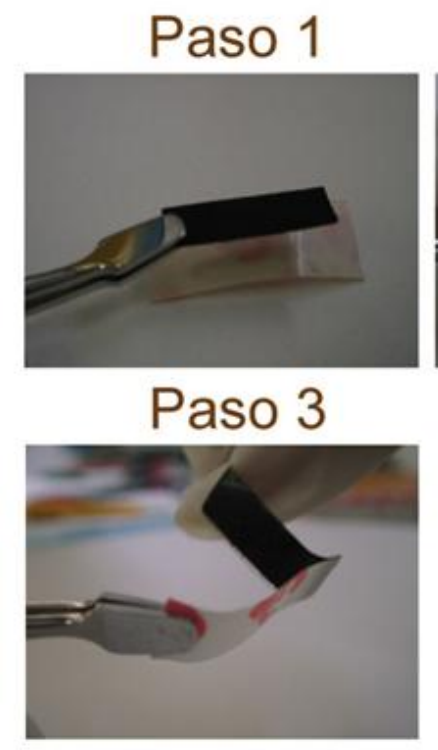
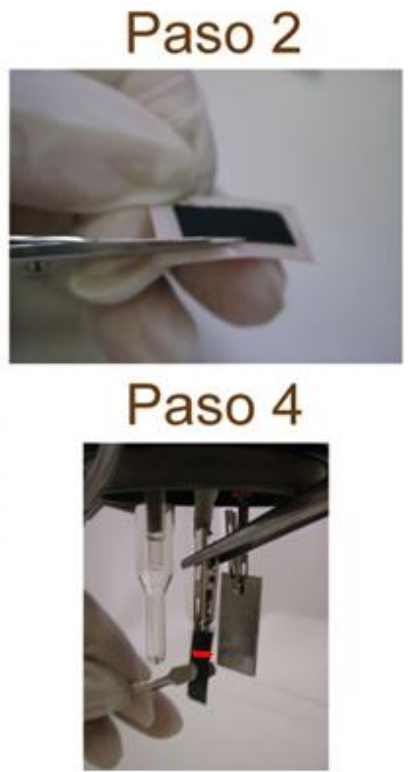

Figura 2.7 Secuencia de armado de un actuador tipo bicapa película//cinta adhesiva

\section{II.7 Construcción del actuador tricapa pPy/DBS//cinta// pPy/DBS}

El actuador de triple capa basado en el material pPy/DBS se fabricó mediante el uso de la misma cinta de doble cara no conductora que fue utilizada para el actuador bicapa, con la diferencia de que una película pPy/DBS se adhiere a cada lado de la cinta siguiendo el mismo procedimiento que para la bicapa. Una de las capas de pPy/DBS está conectada eléctricamente a la salida del electrodo de trabajo del potenciostato-galvanostato. La película de pPy/DBS unida al lado opuesto de la capa adhesiva es conectada a la salida del contraelectrodo y al electrodo de referencia (en cortocircuito) del potenciostato-galvanostato. De esta forma podemos seguir la evolución del potencial del músculo artificial tipo tricapa (potencial del pPy/DBS actuando como electrodo de trabajo frente al potencial de la película de pPy/DBS actuando como contraelectrodo) mientras haya un flujo de corriente. No hay contacto eléctrico (cortocircuito) entre las dos películas de polímero conductor debido a la cinta intermedia adhesiva y no conductora. La Figura 2.8 esquematiza la manera de conectar el actuador tricapa al potenciostato-galvanostato.

Como respuesta a cualquier paso de corriente, el extremo libre del actuador triple capa describe un movimiento angular alrededor del extremo fijo. El seguimiento del movimiento angular se consigue mediante tratamiento de las imágenes tomadas por un sistema de visión descrito en la siguiente sección. 


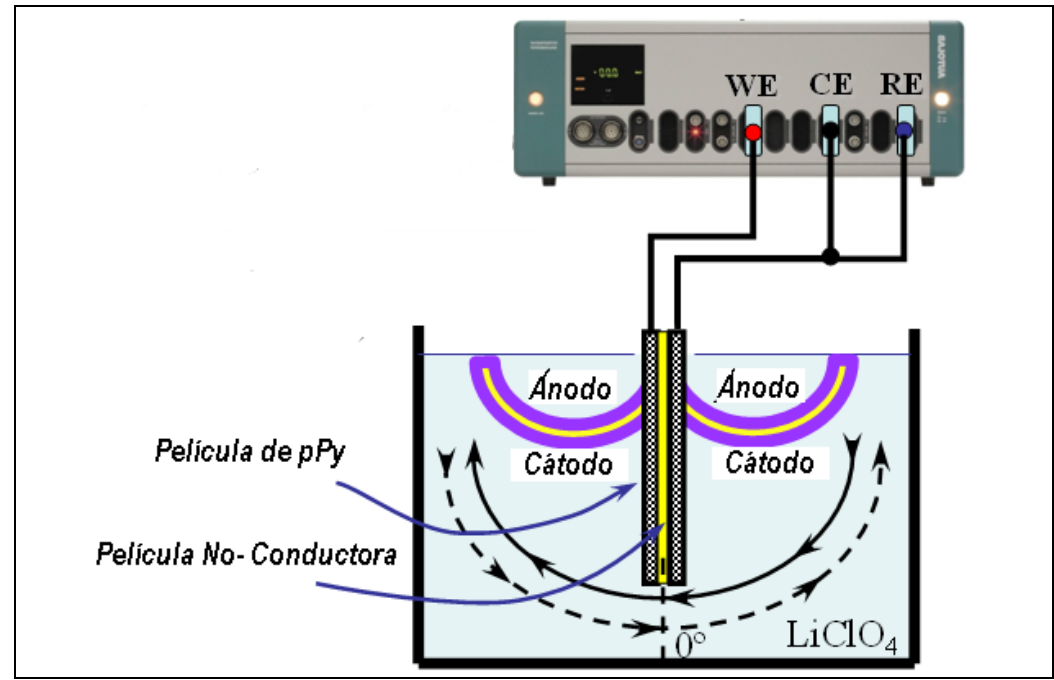

Figura 2.8 Conectividad de un actuador polimérico tricapa.

\section{II.8. Caracterización dinámica de los actuadores bicapa y tricapa}

Para el estudio del movimiento de los actuadores, se utilizó una plataforma de grabación de video con posibilidad de seguimiento tridimensional de los músculos bicapa y tricapa con movimiento angular basado en un medio gráfico cuadriculado como referencia. El equipo consta de 2 cámaras de video de marca Sony EVI-D31, conectadas por una interfase (tarjeta de adquisición de video, MATROX II) a una computadora personal. A este equipo audiovisual se le ha denominado 3D-Grab system, el sistema completo se ilustra en la Figura 2.9. Con ambas cámaras se pueden tomar vídeos de forma ortogonal durante el movimiento de los actuadores y registrarlos de forma sincronizada, entre ellos y con la señal (barrido de potencial, salto de potencial o salto de corriente) aplicada al músculo, con el apoyo de un software denominado 3D-Grab ${ }^{192}$ para controlar y operar las cámaras desde la computadora. Posteriormente se obtuvieron los fotogramas del video con el software InfranView, para posteriormente analizarlos y medir los ángulos de desplazamiento con un programa elaborado ex profeso para ello en Matlab.

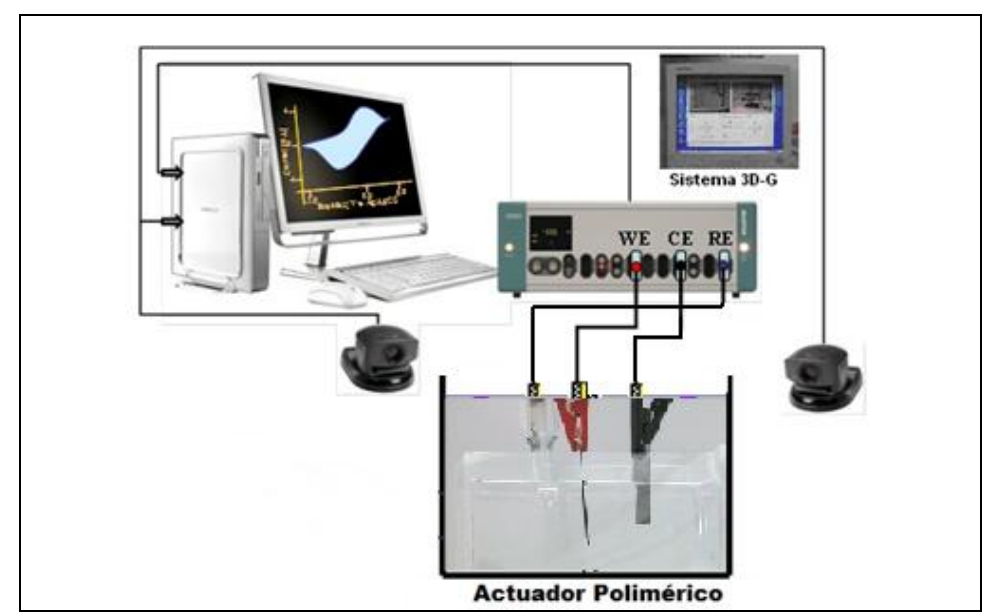

Figura 2.9 Sistema 3D-Grab 


\section{III.1 Caracterización electroquímica Película pPy/DBS autosoportadas}

Para construir músculos artificiales electro-quimio-mecánicos con películas de pPy/DBS es conveniente caracterizar previamente el comportamiento electroquímico del material activo que los componen: las películas de pPy/DBS autosoportadas. Para ello se han aplicado distintas metodologías electroquímicas, estudiando la influencia en cada una de las respuestas obtenidas de las variables químicas y electroquímicas: la velocidad de barrido, el potencial aplicado, la corriente aplicada, la temperatura de trabajo, la concentración del electrolito, el tiempo de reducción o las cargas eléctricas consumidas tanto reversibles como irreversibles.

\section{III.2 Influencia del límite catódico de potencial.}

Una película autosoportada de pPy/DBS, preparada tal y como se describió en la sección experimental, se empleó como electrodo de trabajo en una disolución acuosa de $0.1 \mathrm{M}$ $\mathrm{LiClO}_{4}$. Se comenzó estudiando su comportamiento mediante Voltamperometría Cíclica (VC), con diez barridos consecutivos de potencial a una velocidad de $6 \mathrm{mV} / \mathrm{s}$ entre $-1 \mathrm{~V}$ y $0.8 \mathrm{~V}$, con el fin de estabilizar la película, borrar cualquier memoria estructural del material adquirida durante su geneación y obtener finalmente una respuesta estacionaria: los voltamperogramas consecutivos se solapan. Las respuestas estacionarias del $\mathrm{PPy} / \mathrm{DBS}$ suelen requerir maás de 6 barridos consecutivos. Posteriormente se procedió a estudiar la influencia del límite catódico, manteniendo constante el límite anódico de potencial a $0.6 \mathrm{~V}$, respecto al electrodo de referencia $\mathrm{Ag} / \mathrm{AgCl}$; variando el límite catódico de potencial para cada experimento: $-0.4,-0.6,-0.8,-1,-1.2$ y $-1.4 \mathrm{~V}$. La Figura 3.1 muestra los segundos voltamperogramas de cada experimento superpuestos para los diferentes límites catódicos.

Durante el barrido catódico, se observa un solo pico de reducción del material a - $0.765 \mathrm{~V}$, que se superpone para los diferentes límites catódicos empleados. Sin embargo, durante el barrido anódico, se observa que el pico de oxidación se desplaza hacia potenciales más positivos cuando el material se ha reducido a potenciales cada vez más catódicos. Este tipo de desplazamientos se relaciona en la literatura con cambios estructurales (formación de vacuolas o lamelas) ocasionados por la reacción de reducción dentro de la película polimérica ${ }^{193-196}$. 


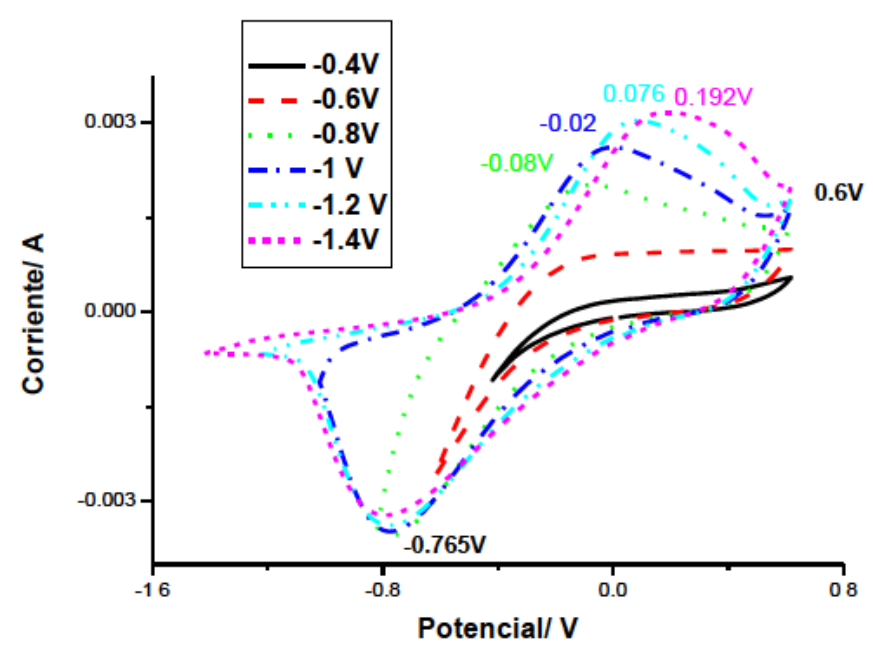

Figura 3.1 Voltamperograma cíclico obtenido de una película pPy/DBS autosoportada a $6 \mathrm{mV} / \mathrm{s}$ entre diferentes límites catódicos $(-0.4,-0.6,-0.8,-1,-1.2,-1.4)$ y un único límite anódico $0.6 \mathrm{~V}$ respecto al electrodo de referencia $\mathrm{Ag} / \mathrm{AgCl}$ en disolución acuosa $0.1 \mathrm{M} \mathrm{LiClO}_{4}$ a temperatura ambiente.

Con el fin de obtener el mayor rango de potencial posible en el que el material es capaz permanece electroactivo, se procedió a abrir la ventana de potencial hasta -2.0 V. En la Figura 3.2 se observa que el pico anódico se desplazó hasta $0.356 \mathrm{~V}$ y el pico catódico hasta $-0.850 \mathrm{~V}$. La separación entre ambos picos es de $494 \mathrm{mV}$, lo cual se atribuye en parte al espesor de las películas ${ }^{197-199}$ y a los cambios estructurales en la película polimérica. La reducción de la película continua después del máximo catódico, aunque a una velocidad mucho menor, pero la corriente nunca cae a cero. Después de su reducción hasta $-2.0 \mathrm{~V}$ la película autosostenida se vuelve a reoxidar, durante el siguiente barrido anódico, en los barridos consecutivos. Este hecho contradice el modelo de transición conductor/aislante durante la reducción de los polímeros conductores que sostiene que los polímeros conductores sólo conducen la electricidad en el estado oxidado, siendo no conductores cuando, al reducirse, pierden la conjugación. Gran parte de los científicos y tecnolólogos (electroquímicos, químicos, físicos, ingenieros y deseñadores de dispossitivos) siguen aplicando este modelo. El modelo de relajación conformacional explica las variaciones estructurales (relajación, hichado, contracción, compactación o formación de vacuolas) que las reacciones electroquímicas de oxidación/reducción originan en el material. Durante la reducción la estructura se abre (máximo de reducción) atrapando gran cantidad (hasta el $40 \%$ ) de los contraiones, con los correspondientes polarones, origen de la alta conductividad del material reducido. 


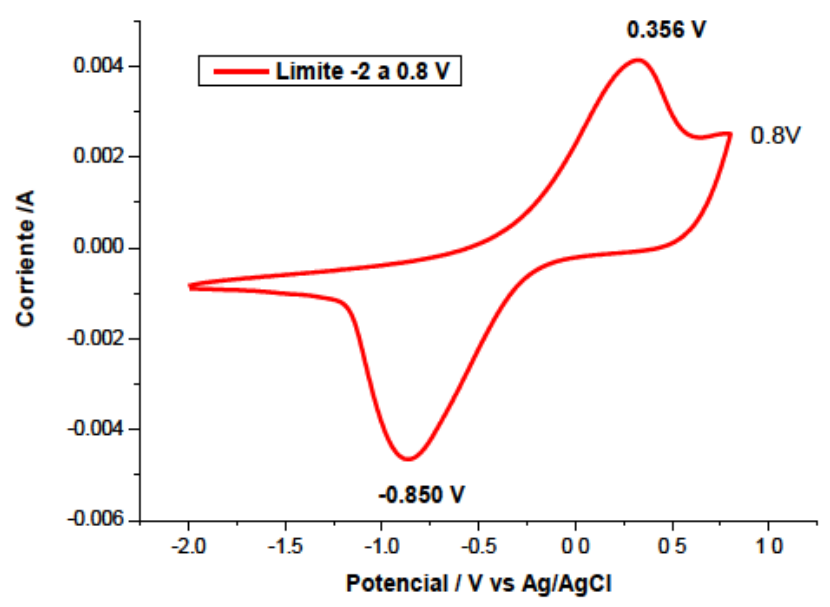

Figura 3.2 Voltamperograma cíclico obtenido de una película pPy/DBS autosoportada a $6 \mathrm{mV} / \mathrm{s}$ en disolución acuosa de $0.1 \mathrm{M} \mathrm{LiClO}_{4}$ a temperatura ambiente.

\section{III.3 Influencia de la velocidad de barrido}

El grado de oxidación y reducción alcanzado en el material por métodos voltamperométricos, está relacionado con la velocidad de barrido (tiempo disponible para oxidar/reducir el polímero) debido a la presencia de limitaciones cinéticas durante la reacción electroquímica. Dicho grado de oxidación/reducción tendrá un efecto en las propiedades del material (y dispositivos basados en él).

Las respuestas voltamperométricas estacionarias (alcanzadas después de de dos o tres voltamperogramas consecutivos) obtenidas de una película autosoportada de $\mathrm{pPy} / \mathrm{DBS}$, a diferentes velocidades de barrido indicadas en la figura, se presentan en la Figura 3.3. A velocidades inferiores a $10 \mathrm{mV} / \mathrm{s}$ los máximos de oxidación y reducción aparecen bien definidos. En el intervalo de velocidades de barrido de $20 \mathrm{mV} / \mathrm{s}$ a $50 \mathrm{mV} / \mathrm{s}$ se aprecia que los procesos redox son incompletos. 


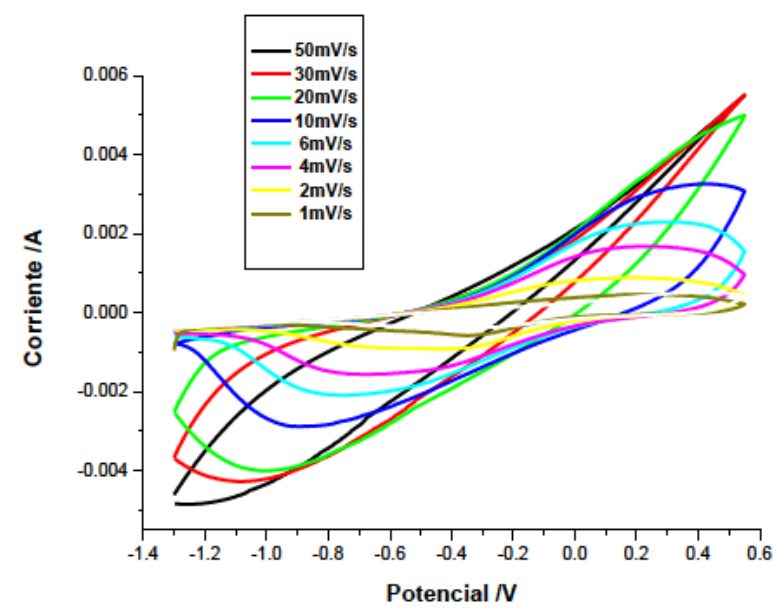

Figura 3.3 Voltamperogramas cíclicos entre +0.55 y $-1.3 \mathrm{~V}$ respecto al electrodo de referencia Ag/AgCl obtenidos de una película pPy/DBS autosoportada a diferentes velocidades de barrido de $50 \mathrm{mV} / \mathrm{s}$ a $1 \mathrm{mV} / \mathrm{s}$ en disolución acuosa $0.1 \mathrm{M} \mathrm{LiClO}_{4}$ a temperatura ambiente.

En la Figura 3.4 se observa que la intensidad de los picos voltamperométricos de la Figura 3.3 (los que se observan bien definidos, para velocidades de $10 \mathrm{mV} / \mathrm{s}$ o menores) se incrementa como una función de la raíz cuadrada de la velocidad de barrido, lo que confirma que en este intervalo de potenciales, el proceso de oxidación/reducción se produce bajo el control de difusión de los iones $\left(\mathrm{Li}^{+}\right)$dentro de la película polimérica ${ }^{183}$. A velocidades mayores de $20 \mathrm{mV} / \mathrm{s}$, la forma resistiva del voltamperograma indica que aparecen limitaciones de transporte iónico requerido para balancear las cargas en la película y mantener así la electroneutralidad.

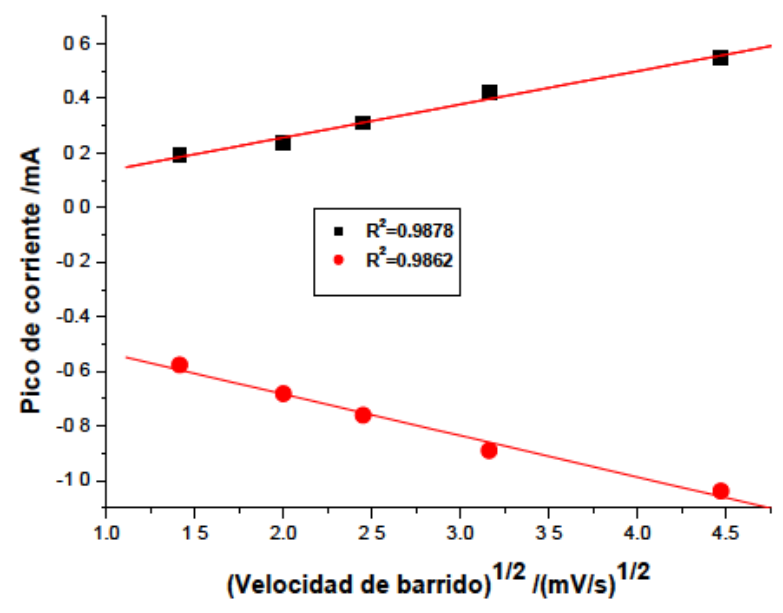

Figura 3.4 Raíz cuadrada de la velocidad de barrido de una película pPy/DBS en $\mathrm{mV} / \mathrm{s}$ frente al máximo medido de corriente en $\mathrm{mA}$. Valores obtenidos de la Figura 3.3. 


\section{4 Reversibilidad, estabilidad en películas autosoportadas pPy/DBS}

Para poder conocer el tiempo de vida que puede llegar a tener un dispositivo es importante conocer aspectos del material tales como la estabilidad de su comportamiento o si va a responder siempre igual durante diferentes ciclos de vida. Las respuestas voltamperométricas a una velocidad de barrido de $4 \mathrm{mV} / \mathrm{s}$ con diferentes límites de potencial extremos se muestran en la Figura 3.5. Se observa en el voltamperograma del primer ciclado (línea continua y color negro) en la ventana electroquímica $-2.5 \mathrm{a}+2.5 \mathrm{~V}$, un pico catódico a $-0.752 \mathrm{~V}$ y un anódico a $0.418 \mathrm{~V}$ que es comparable a los resultados obtenidos anteriormente en la Figura 3.2. A potenciales mayores de 1V la corriente crece exponencialmente hasta $19 \mathrm{~mA}$. En el segundo barrido consecutivo utilizando la misma ventana electroquímica $(-2.5 \mathrm{a}+2.5 \mathrm{~V})$ ), se observa (Figura 3.5 línea punteada de color rojo) un desplazamiento del pico anódico a $1.93 \mathrm{~V}$ con un pequeño aumento en la corriente. El pico catódico se ha desplazado a $-0.51 \mathrm{~V}$ a la vez que presenta un descenso en la corriente de -2 mA: el material se ha vuelto más resistivo debido a que la reducción requiere mayor sobretensión. En películas de polipirrol con aniones inorgánicos pequeños los barridos hasta sobretensiones anódicas tan altas producen la sobreoxidación del material, que pierde totalmente su electroactividad en el segundo barrido ${ }^{190}$ Aquí el pPy/DBS vemos que solo sufre una degradación parcial de su electroactividad (carga total consumida durante su oxidación/reducción reversible) durante el primer barrido.

Al abrir la ventana de potenciales entre $-3 \mathrm{Va}+3 \mathrm{~V}$ (voltamperograma de línea azul puntoraya), se observa que el material se recupera y presenta 2 máximos de oxidación, rama anódica. El máximo a $0.76 \mathrm{~V}$ y $3 \mathrm{~mA}$, es muy similar al encontrado en el primero de los barridos (negro). Por encima de 1.2V no se observa descarga de oxígeno, la corriente sube hasta formar un pico a $2.81 \mathrm{~V}$ con una corriente de $22 \mathrm{~mA}$. Aquí, de forma contraria a lo que se ha reportado para polímeros conductores que intercambian aniones, durante el barrido catódico el voltamperograma presenta el inicio de la reacción de reducción del material a $-0.5 \mathrm{~V}$ con un pico de reducción a $-1.31 \mathrm{~V}$ con una corriente de $-3 \mathrm{~mA}$. $\mathrm{A}$ potenciales más catódicos no se observa el aumento indefinido de la corriente propio de la descarga de hidrógeno, por lo que se podría asumir que el material puede ser un fuerte inhibidor de hidrógeno. Además, el material mantiene una alta estabilidad electroquímica (electroactividad) a pesar de someterse a muy elevados potenciales catódicos y anódicos en los sucesivos ciclos, por lo que podría ser utilizado en condiciones de potencial extremas sin la pérdida total de electroactividad. 


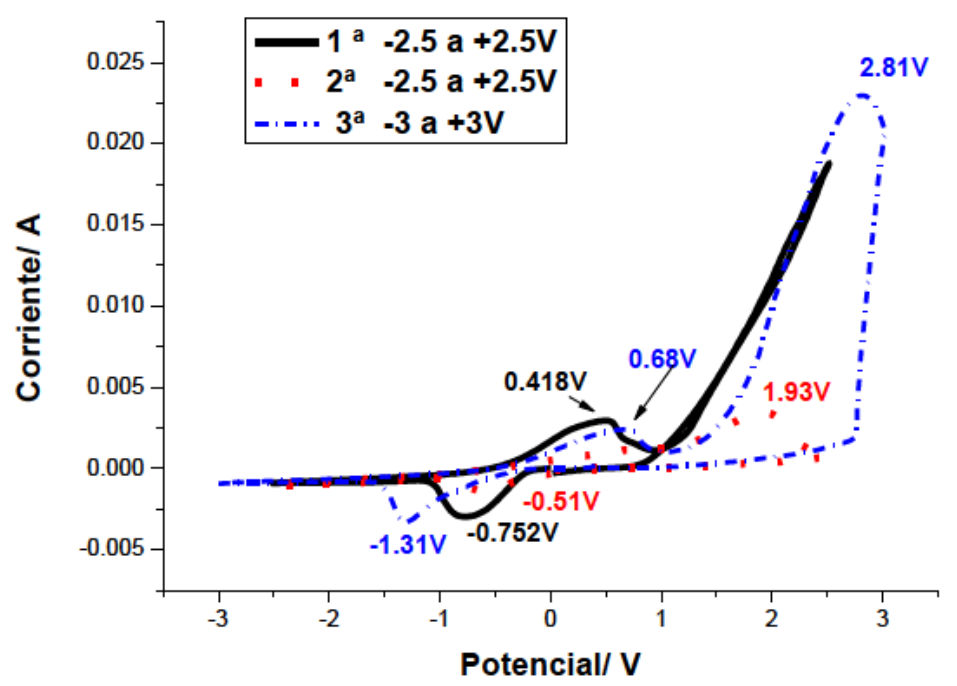

Figura 3.5 Voltamperograma cíclico obtenido de una película autosoportada $p P y / D B S$ a $4 \mathrm{mV} / \mathrm{s}$ en una disolución acuosa $0.1 \mathrm{M} \mathrm{LiClO}_{4}$ a temperatura ambiente, en los intervalos de potenciales $-2.5 \mathrm{a}$ +2.5 (primer y segundo ciclado) y de -3 a +3 V (tercer ciclado). La línea continua, indica el primer ciclo la línea punteada en rojo el segundo ciclo y la línea punteada en azul el tercer ciclo.

\section{III.5 Respuestas culovoltamétricas}

Por integración de las respuestas voltamperométricas obtenidas hasta distintos potenciales catódicos (Figura 3.6 a) se obtiene las respuestas culovoltamétricas, (Figura 3.6 b): carga que ha pasado por el electrodo respecto al potencial aplicado. Los culovoltagramas han resultado una herramienta poderosa para estudiar la reversibilidad de las reacciones electroquímicas. En ellos, los incrementos positivos de carga indican oxidación de la película, los negativos, reducción ${ }^{200}$. Los culovoltagramas obtenidos para películas autosoportadas pPy/DBS (Figure 3.6 b) presentan un bucle cerrado y una parte abierta a su izquierda. El bucle cerrado (carga de oxidación igual a la carga de reducción) representa la carga invertida en la oxidación/reducción reversible de la película de pPy/DBS. La parte abierta es una carga consumida en reacciones irreversibles no recuperándose con el ciclado.

A pesar de no observarse un incremento de la corriente en los voltamperogramas a potenciales muy catódicos, esta carga irreversible podría estar relacionada con la evolución de hidrógeno, simultaneamente a la reducción del polímero, a potenciales catódicos mayores a $-1.1 \mathrm{~V}^{200}$. Hay que destacar que la presencia de la liberación de hidrógeno no produce un aumento de las corrientes catódicas al aumentar el potencial catódico en los voltamperogramas. El cómo y dónde tiene lugar esta evolución de 
hidrógeno sigue siendo una cuestión pendiente para futuros estudios. El hecho de que en las películas autosoportadas de polipirrol con pequeños iones inorgánicos no se produzca esta carga irreversible hasta potenciales mas catódicos que $-2.5 \mathrm{~V}$ en disolución acuosa apunta al DBS, y en particular a su foma ácida HDBS, como origen de esta descarga.
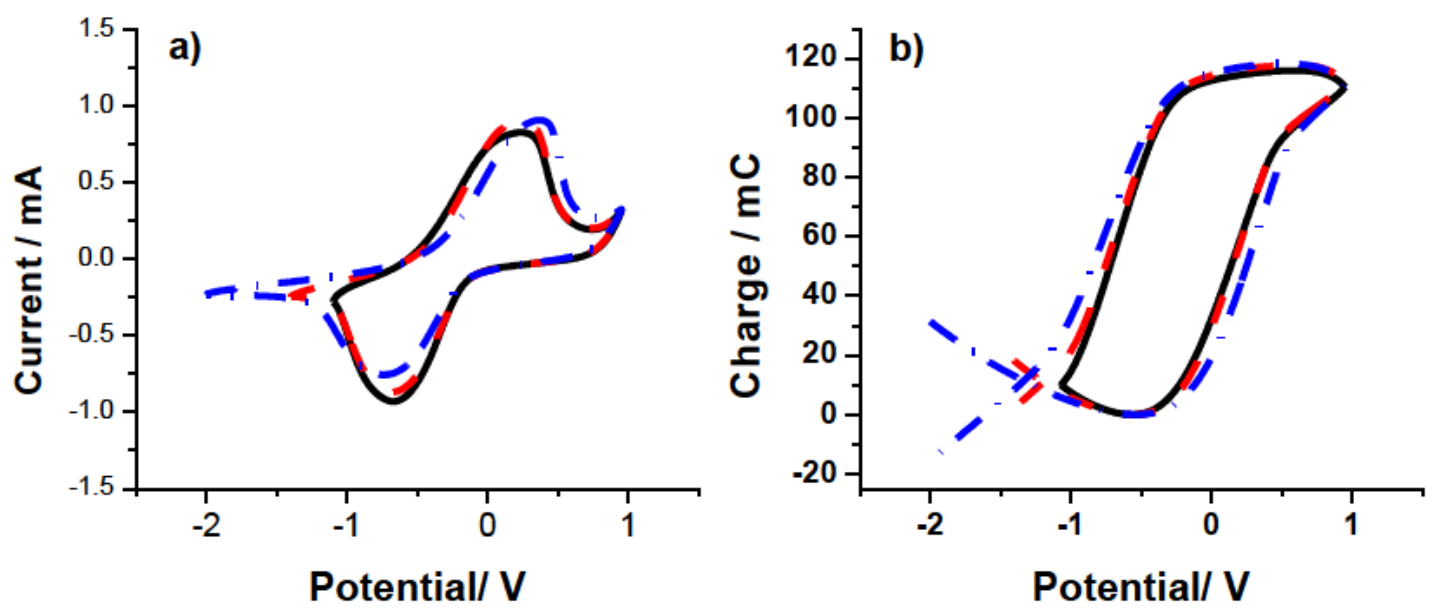

Figura 3.6. Respuestas (a) Voltamperométricas y (b) culovoltamétricas de una película pPy/DBS autosuportada hasta diferentes potenciales catódicos $(-1,-1.5$ y $-2 \mathrm{~V})$ en disolución acuosa $0.1 \mathrm{M}$ de $\mathrm{LiClO}_{4}$. A una velocidad de barrido $6 \mathrm{mV} \mathrm{s}^{-1}$.

Cualquier variación en la pendiente $Q / V$ de los culovoltagramas indica un cambio de la velocidad de la reacción ${ }^{201}$.

En la Figura 3.7 se observa un culovoltagrama obtenido en las mismas condiciones experimentales de la figura 3.6, pero hasta un potencial catódico todavía mayor (-2.5 V). En él se observa que la pendiente carga-potencial presenta distintas e importantes varíaciones, indicando que la cinética de las reacciones de oxidación y de reducción de la película varía a lo largo del barrido de potencial. Debido al intercambio de cationes durante las reacciones para mantener la electroneutralidad, el material se contrae durante la oxidación, se cierra la estructura cuando la distancia media entre las cadenas se hace igual al diámetro de los contraiones atrapando hast aun $40 \%$ de los cationes y posteriormente se empaqueta con expulsión de cationes y disolvente, de acuerdo al modelo de relajación conformacional estimulada electroquímicamente (ESCR, siglas en inglés) ${ }^{202-211}$. Durante la reducción el material relaja su estructura compactada par permitir el inicio de la entrada de cationes impulsada por la reacción, lugo se hincha con entrada de cationes y agua, siguiendo la reacción (1.2). Las respuestas culovoltamétricas (Figura 
3.7), revelan durante el barrido anódico el paso desde una oxidación-contracción rápida a una oxidacción-empaquetamiento lenta a potenciales altos: el potencial de cambio de pendiente es el potencial de cierre. La reducción empieza con una relajación lenta de la estructura empaquetada (evolución muy lenta de la carga de reducción por unidad de voltaje), seguido de un proceso rápido de reducción-hinchamiento. Sin embargo, para una reducción profunda (curva azul punteada) más allá de $-1.2 \mathrm{~V}$ la reducción se vuelve lenta de nuevo. Esto apunta a la reducción con formación de lamelas, estructuras tipo micela o vacuolas dentro del material debido a la naturaleza del DBS, según lo propuesto en trabajos anteriores ${ }^{193,194}$. Este nuevo aspecto estructural y su cuantificación requerirá de un estudio profundo posteriormente.

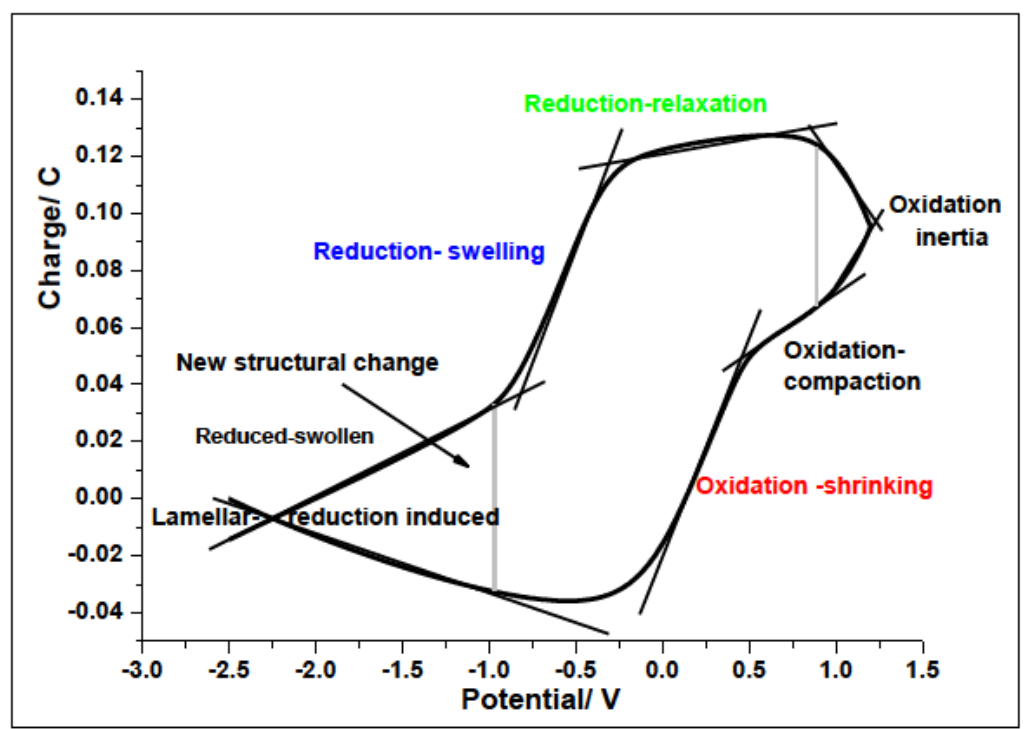

Figura 3.7. Respuesta culovoltamétrica obtenida de una película autosoportada pPy/DBS en disolución acuosa $0.1 \mathrm{M}$ de $\mathrm{NaCl}$ a velocidad de barrido de $6 \mathrm{mV} / \mathrm{s}$, a temperatura ambiente. Muestra las variaciones abruptas de pendiente relacionadas con las variaciones bruscas en la velocidad de reacción ( $d Q / d E=1 / F m d[A-] / d E$ ). Dichos cambios son debidos a las cuatro transiciones estructurales dentro de la película, manejados por las reacción y descrito por el modelo ESCR: oxidación-reducción, oxidación-compactación, reducción-relajación y reducciónhinchazón. Además la película polimérica presenta una nueva variación estructural lenta en el estado reducido-hinchado que puede ser debido a una organización micelar o lamelar del DBS y agua. 


\section{6 Pérdida de electroactividad en películas autosoportadas pPy/DBS (tiempo de}

vida)

Se estudió la pérdida de electroactividad (degradación) de películas autosoportadas pPy/DBS mediante voltamperometría cíclica sometiendo a la película a 50 barridos consecutivos a una velocidad de $6 \mathrm{mV} / \mathrm{s}$ en las mismas condiciones experimentales.

En la Figura 3.8 se presentan los voltamperogramas de una película autosoportada pPy/DBS de $72 \mu \mathrm{m}$ de espesor, peso de $6.38 \mathrm{mg}$ y un área de $0.88 \mathrm{~cm}^{2}$ dentro de la disolución. El área dentro de la disolución, el área total de la película y su peso total permiten obtener el peso de película dentro de la disolución, Se observa un desplazamiento de los picos, haciéndose cada vez más importante la diferencia entre los potenciales de los picos anódicos y catódicos (mayor resistividad de la película), a la vez que se observa un descenso en la corriente de pico.

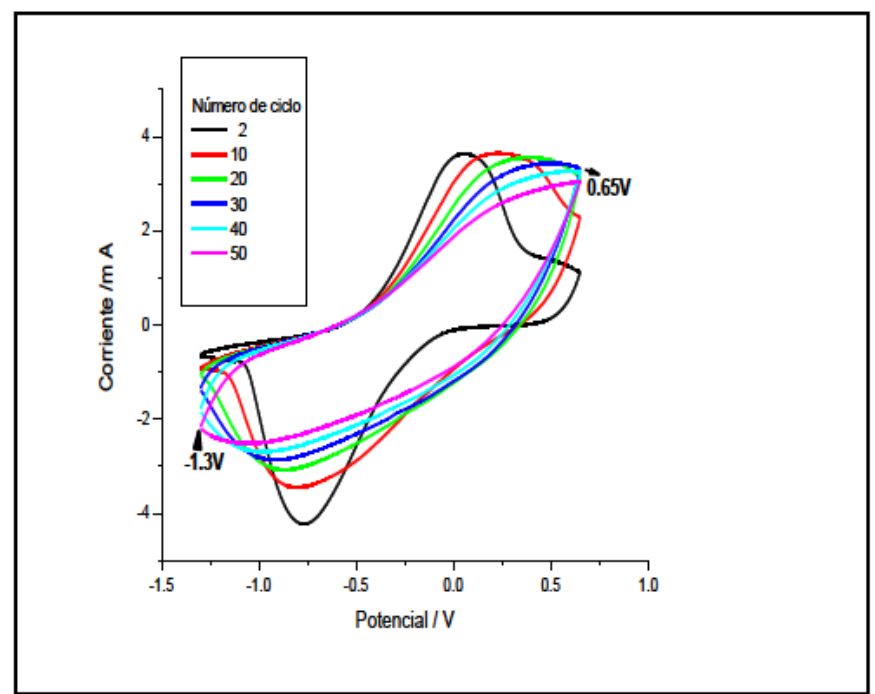

Figura. 3.8 Voltamperogramas obtenidos de una película autosoportada pPy/DBS entre $-1.3 \mathrm{~V} y$ $0.65 \mathrm{~V}$ a una velocidad de $6 \mathrm{mV} / \mathrm{s}$ en disolución acuosa $0.1 \mathrm{M} \mathrm{LiClO}_{4}$ a temperatura ambiente durante diferentes ciclos (se muestran los ciclos 2,10,20,30,40 y 50).

Representando la relación de números de ciclo frente a la carga eléctrica específica (por mg de material) consumida se obtiene una disminución de la electroactividad del material, presentando un comportamiento lineal la relación de la carga eléctrica con el número de ciclos de potencial, como se observa en la Figura 3.9: la electroactividad del material se degrada linealmente con el ciclado, es decir, en cada ciclo se observa una degradación similar. 


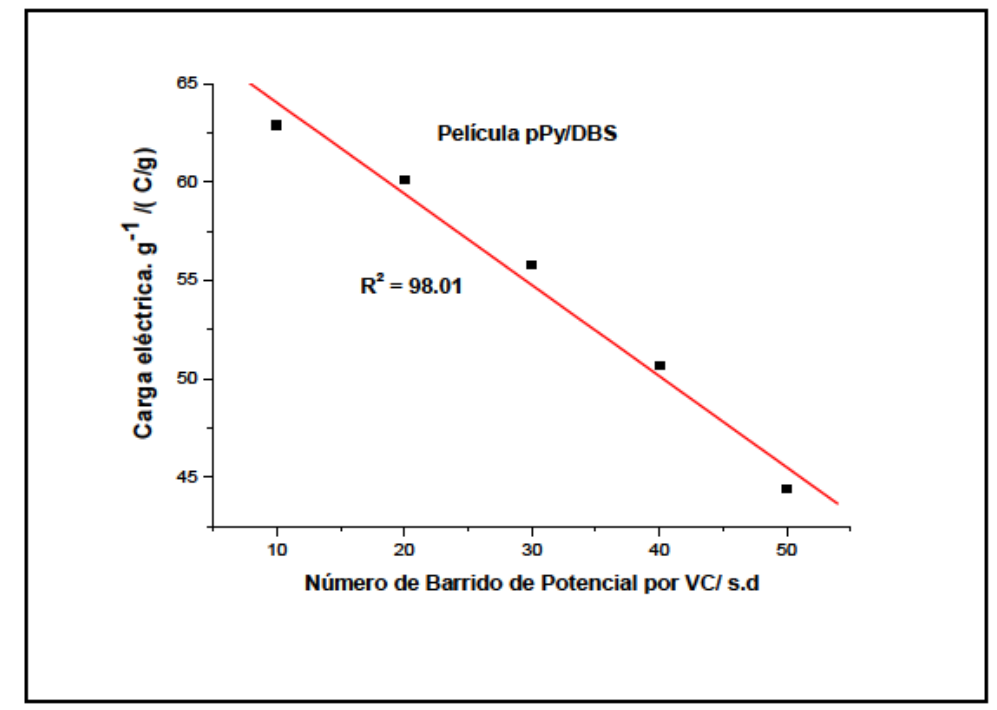

Figura 3.9 Relación de la carga eléctrica especifica consumida durante los voltamperogramas mostrados en la figura 3.8 con el número de ciclo.

\section{III.7 Influencia del tiempo de reducción}

Las figuras 3.2 y 3.6 muestran que la reducción del material sigue, a menor corriente, después del máximo de reducción. La figura 3.7 corobora con un fuerte cambio de pendiente esta disminución de la velocidad de reducción. A continuación se va estudiar cómo afecta la reducción en esa zona de potenciales a la posterior oxidación del material. Pare ello se empleará la cronoamperometría. Se fijó el potencial catódico a $-1.2 \mathrm{~V}$, variando los tiempos en los que la película autosoportada $\mathrm{pPy} / \mathrm{DBS}$ se reducía a dicho potencial de 1 a 1000 segundos. A continuación de cada reducción se saltó el potencial a $0.4 \mathrm{~V}$ durante el tiempo necesario para la completa oxidación del material. La Figura 3.10 muestra que en los cronoamperogramas de oxidación obtenidos tras una prepolarización a tiempos cortos (1-10 s) presentan un máximo muy estrecho seguido de una exponencial decreciente, lo cual es típico de la carga de una doble capa seguido por la oxidación de la película de polímero bajo control de cinética difusional de los contraiones en el interior del polímero ${ }^{211}$. Sin embargo, para tiempos de prepolarización más largos se forma un máximo bien definido, que se desplaza hacia tiempos de oxidación mayores cuando el tiempo de prepolarización catódica aumenta. Estos máximos son característicos de procesos que se producen bajo oxidación-relajación conformacional y nucleación, y han sido descritos para intercambio mayoritario de aniones durante la oxidación/reducción del polímero, por el modelo ESCR $204,205,208$. 


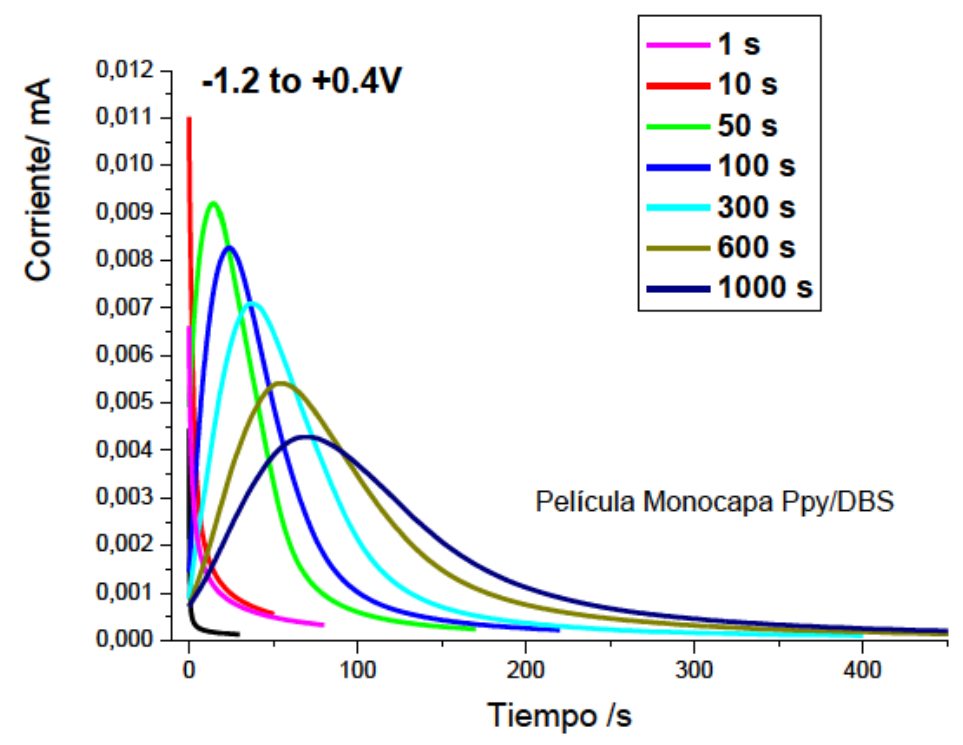

Figura 3.10 Registros cronoamperométricos obtenidos de una película pPy/DBS en una disolución acuosa $0.1 \mathrm{M} \mathrm{LiClO}_{4}$ obtenidos aplicando $0.4 \mathrm{~V}$ tras una pre-polarización a un potencial catódico de $-1.2 \mathrm{~V}$ durante diferentes tiempos.

Una dependencia doble logarítmica entre el tiempo al que se da el máximo flujo de corriente durante la oxidación $\left(t_{\max }\right)$ y el tiempo de reducción $\left(t_{c}\right)$ se observa en la Figura 3.11, con una pendiente de 0.64. Esta misma relación ha sido descrita para películas de pPy y polianilina (PANI) sobre electrodos de platino y ha sido interpretada en términos del modelo ESCR 208, 211-213

Los procesos de relajación y migración son muchos más lentos que los procesos de difusión, donde los contraiones tienen que abrirse camino para atravesar la matriz polimérica empaquetada por estimulación de movimientos conformacionales de la cadena polimérica. 


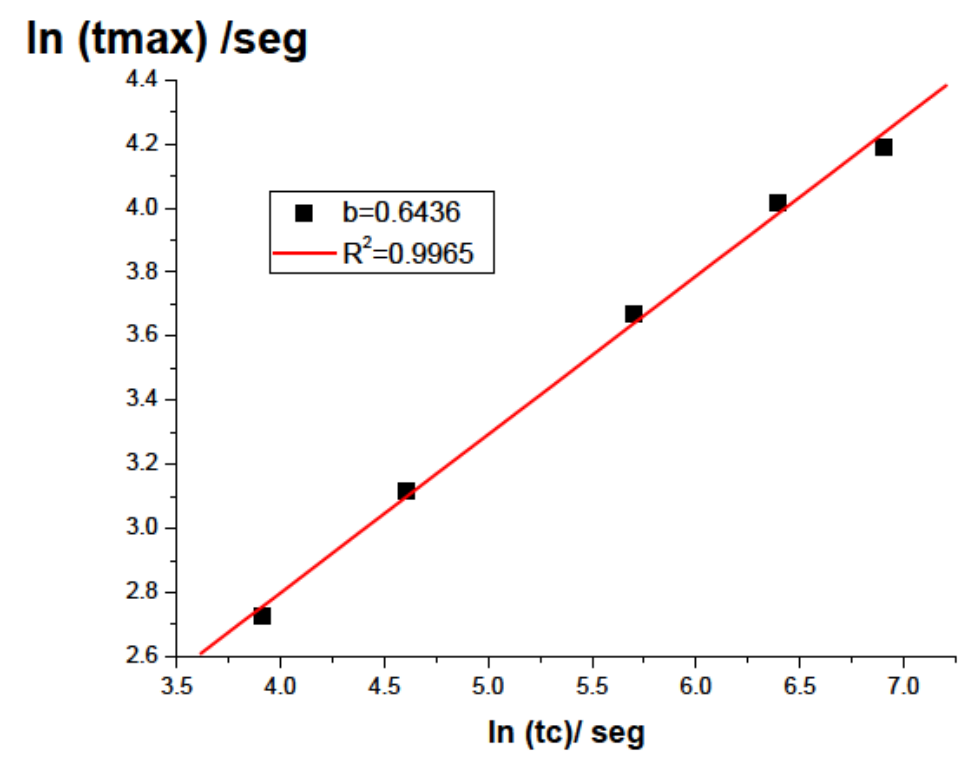

Figura 3.11 Representación doble logarítmica del tiempo a la máxima corriente (tmax) obtenido en la figura 3.10 y el tiempo de prepolarización

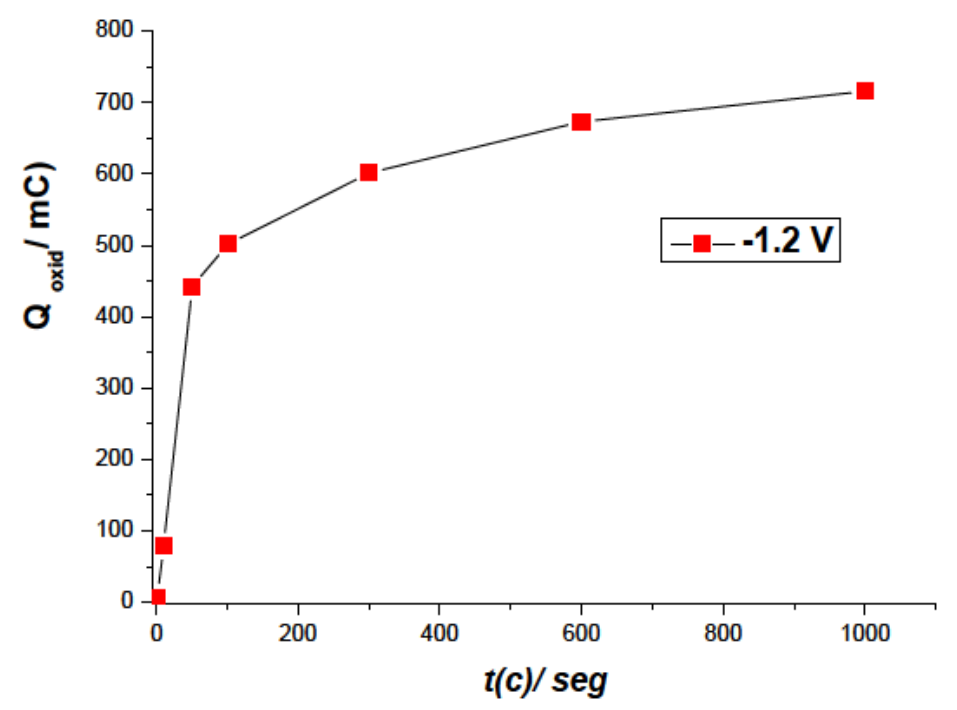

Figura 3.12 Carga de oxidación $\left(Q_{o x i d}\right)$ determinada de los registros cronoamperométricos de película pPy/DBS (Figura 3.10) en función del tiempo de prepolarización t(c) a -1.2 V.

En la Figura 3.12 se muestra la carga de oxidación $\left(Q_{o x i d}\right)$ consumida por la película de pPy/DBS (Figura 3.10) durante la respuesta a los saltos de potencial desde -1.2 a $0.4 \mathrm{~V}$ frente al tiempo de pre-polarización $\mathrm{t}(\mathrm{c}) \mathrm{a}-1.2 \mathrm{~V}$. Se observa que la carga se incrementa, rápidamente el principio, al aumentar el tiempo de pre-polarización hasta alcanzar un 
incremento constante para tiempos de reducción mayores que 300 segundos. Teniendo en cuenta el modelo ESCR, estos resultados parecen indicar un cambio estructural que hace más lenta la llegada de los cationes que han de balancear las cargas del DBS ${ }^{-}$hasta el lugar donde se encuentran estos aniones en el interior del material. La reducción completa del material, de igual forma a como ocurría con los polímeros intercambiadores de aniones ${ }^{200,212,213}$, resulta ser un proceso tanto más lento cuanto mayor es el estado de reducción del material.

\section{8 Corriente aplicada}

Utilizando una metodología experimental ampliamente utilizada en el estudio de dispositivos actuadores/sensores ${ }^{214}$ y como se describió en el capítulo II (Figura 2.5), las películas de pPy/DBS autosoportadas fueron sometidas a ondas cuadradas consecutivas de corriente, aplicando la misma carga de oxidación y de reducción, con el fin de mantener constantes los estados inicial y final de oxidación/reducción. Después de dos ondas cuadradas se obtuvieron las respuestas cronopotenciométricas estacionarias (evolución del potencial).

Los cronopotenciogramas en estado estacionario normalizados (el origen se traslada en cada caso al cero de corriente) obtenidos al someter la película de pPy/DBS a diferentes corrientes, desde \pm 1.5 a $\pm 24 \mathrm{~mA}$, en disolución acuosa $0.1 \mathrm{M} \mathrm{LiClO}_{4}$ durante el tiempo requerido en cada caso para consumir una carga constante de $180 \mathrm{mC}$, se muestran en la Figura 3.13. La gráfica muestra que para mayores corrientes aplicadas, se produce un mayor salto inicial en el potencial, así como este evoluciona a valores más altos más rápidamente (con una mayor pendiente).

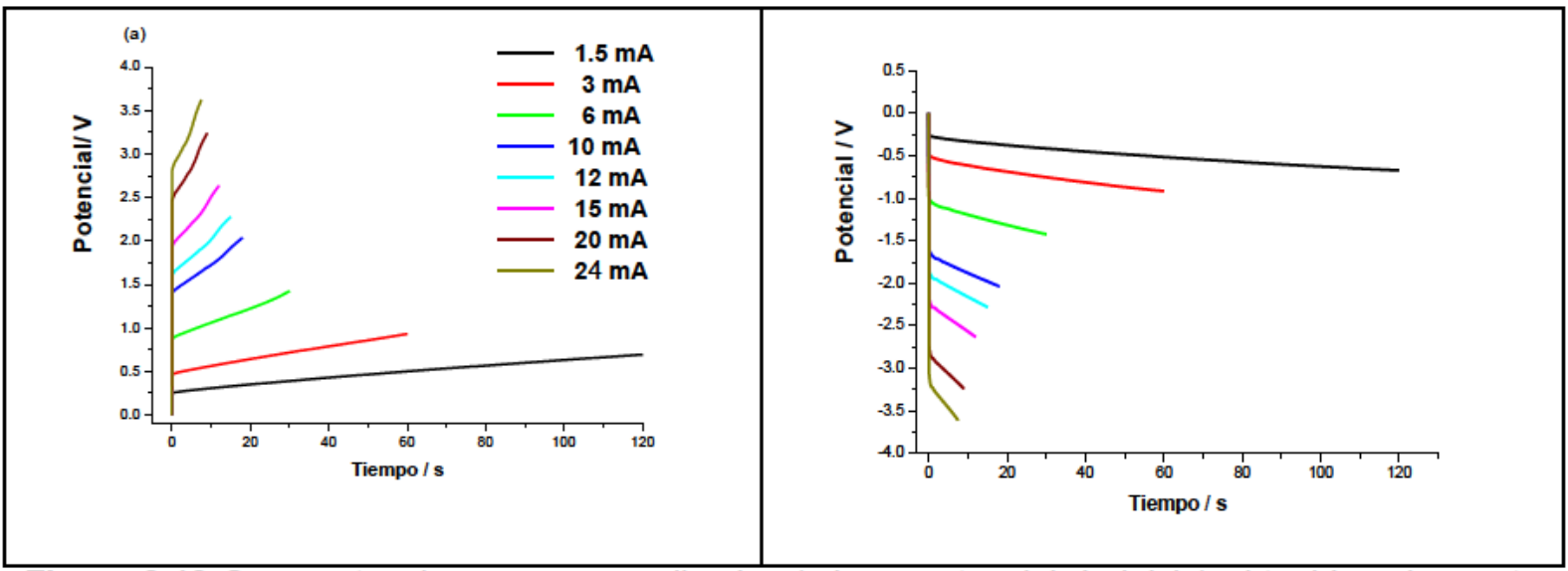

Figura 3.13 Cronopotenciogramas normalizados (mismo potencial de inicio) obtenidos al someter la película de $p P y / D B S$ a ondas cuadradas consecutivas de corrientes, desde \pm 1.5 a $\pm 24 \mathrm{~mA}$, durante el tiempo requerido en cada caso para consumir una carga constante de $180 \mathrm{mC}$, en solución acuosa $0.1 \mathrm{M}$ de $\mathrm{LiClO}_{4}$ y temperatura ambiente. 
Por integración de estas respuestas y multiplicando el valor de dicha integral por la corriente es posible obtener la energía consumida o la evolución de la energía consumida con el tiempo (ecuación 2.3). Si se conoce el peso del polímero reaccionando dentro de la disolución se pueden obtener las energías específicas consumidas durante la oxidación o la reducción (por unidad de peso del polímero).

La variación de la energía eléctrica consumida en función de la corriente aplicada se muestra en la Figura 3.14. Se obtuvo un buen ajuste lineal tanto para los procesos catódicos como para la energía consumida durante los procesos anódicos. Este resultado demuestra la capacidad sensora del material relacionada a la corriente aplicada. Al aumentar la corriente eléctrica aumenta de forma lineal la energía eléctrica consumida, de igual forma a como ha sido reportado anteriormente para actuadores electroquimiomecánicos bicapa o tricapa basados en polímeros conductores de intercambio aniónico ${ }^{214,215}$.

Como conclusión parcial podemos establecer que la energía consumida por la reacción del material pPy/DBS es un buen sensor de las condiciones eléctricas de trabajo. Al ser una propiedad del material, esta capacidad sensora es de esperar que pueda extenderse a los diferentes dispositivos electroquímicos que estén constituidos por el material y trabajen impulsados por la reacción electroquímica del mismo.
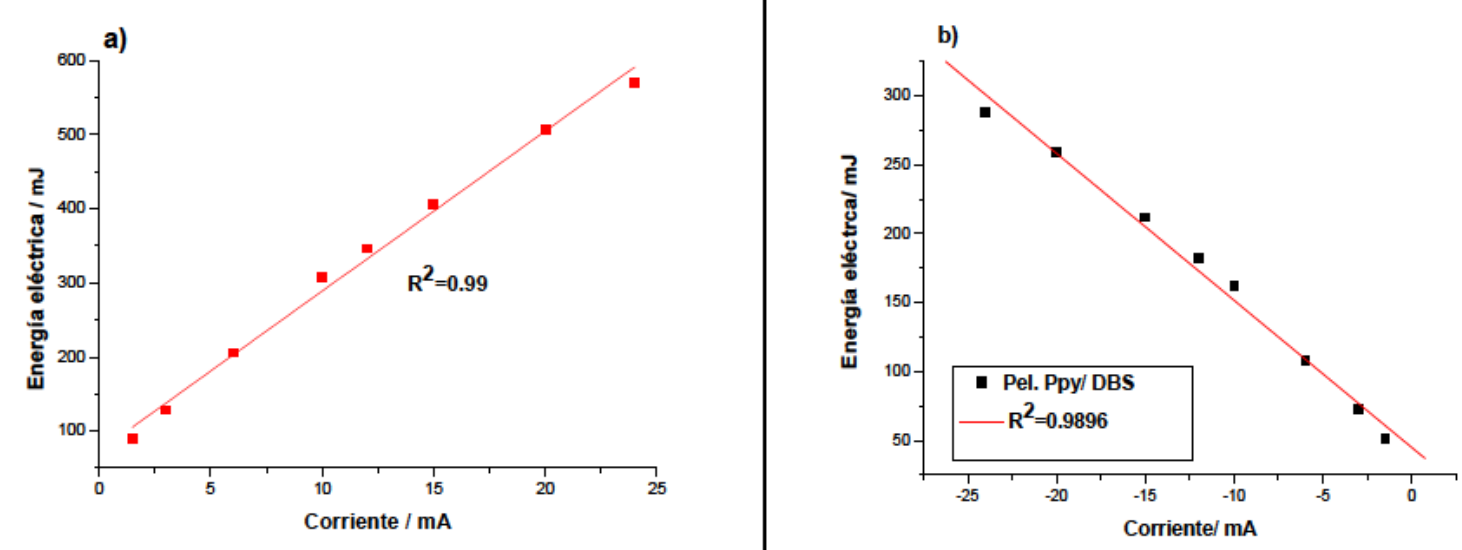

Figura 3.14 Corriente eléctrica consumida de una película de pPy/DBS a) anódica, b) catódica durante los cronopotenciogramas mostrados en la Figura 3.13. 


\section{III.9 Influencia de la temperatura}

De forma similar a cómo se estudió la influencia de la corriente aplicada, se siguieron las respuestas de las películas poliméricas autosoportadas a diferentes temperaturas de trabajo. El objetivo es comprobar la capacidad del material para sentir las condiciones térmicas de trabajo durante la reacción.

Para estudiar la influencia de la temperatura se utilizó un Criostato/ Termostato marca Julabo Easy Temp Professional Modelo T25 $\left( \pm 0.1^{\circ} \mathrm{C}\right)$. El medio de refrigeración fue un líquido anticongelante. La celda electroquímica se introdujo, mediante un adaptador, para dentro del baño. En el interior de la celda se introdujo un termómetro para poder así determinar la temperatura del electrolito de trabajo.

Una vez estabilizada la temperatura a un diferente valor cada vez $(15,20,25,30,35$ y 40 $\circ$ C), se aplicó el procedimiento experimental descrito en la Figura. 2.5. La corriente seleccionada fue de $\pm 0.8 \mathrm{~mA}$ durante $60 \mathrm{~s}$, tanto la anódica, como la catódica.

La Figura 3.15 muestra, superpuestas y normalizadas, las respuesta cronopotenciométricas estacionarias de la película al flujo de la rama de corriente anódica (Figura 3.15 a) y al de la rama catódica (Figura. 3.15b) de las ondas cuadradas aplicadas a diferentes temperaturas. Para ambos procesos (a) anódicos y b) catódicos), al aumentar la temperatura se observa, para los mismos tiempos de flujo de corriente, una disminución de los potenciales del material. El aumento de la energía térmica disponible durante la reacción requiere un menor consumo de energía eléctrica para que transcurra la reacción del material entre los mismos estados de oxidación inicial y final. Por consiguiente, siendo la energía eléctrica $\mathrm{EQ}=$ Eit, para flujo de la misma corriente (i) durante el mismo tiempo $(\mathrm{t})$, el potencial del material evoluciona a valores menores. 

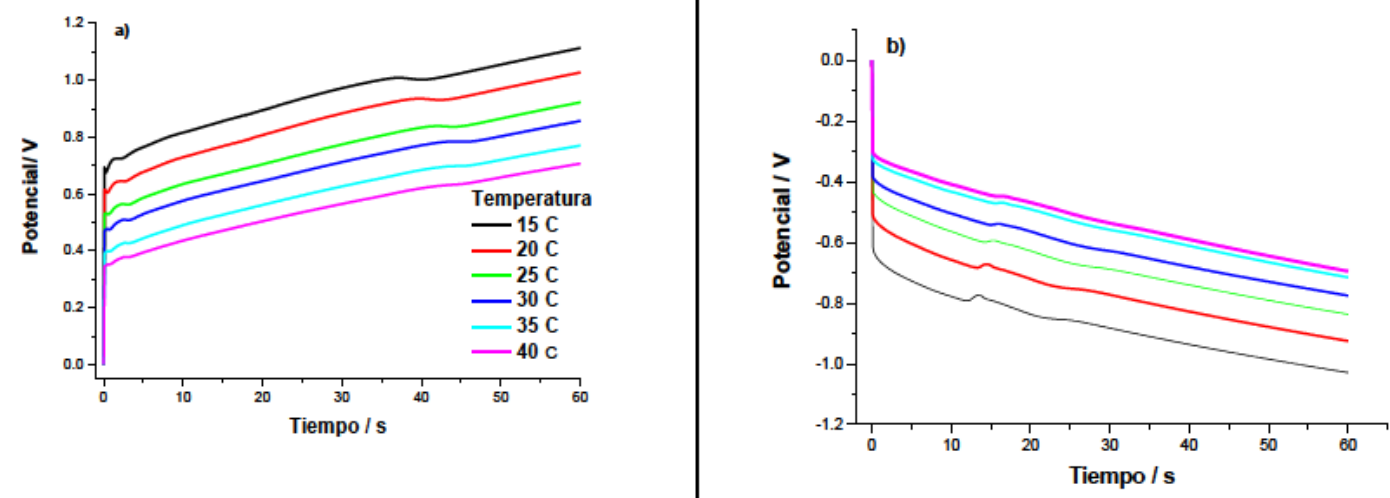

Figura 3.15 Cronopotenciogramas normalizados (mismo potencial inicial) obtenidos de una película autosoportada $p P y / D B S$ a diferentes temperaturas cuando: a) $0.8 \mathrm{~mA}$ y b) $-0.8 \mathrm{~mA}$ se aplican a la película 60 s en disolución acuosa $0.1 \mathrm{M} \mathrm{LiClO}_{4}$ a temperatura ambiente.

La Figura 3.16 muestra la energía eléctrica específica (por g de material) consumida, la cual disminuye linealmente, tanto para la oxidación como para la reducción del material entre los mismo estados de oxidación inicial y final, al aumentar la temperatura de trabajo.

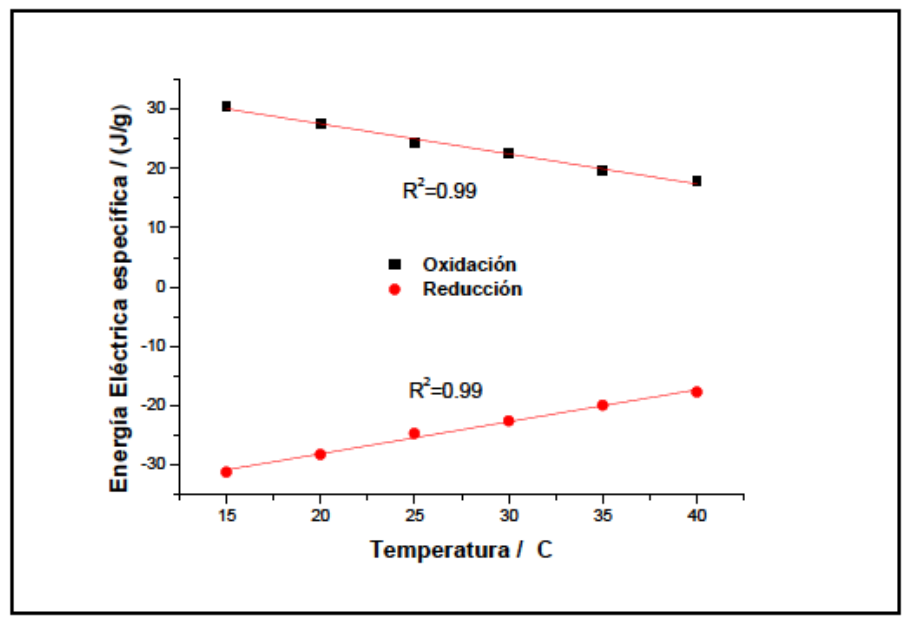

Figura 3.16: Energía eléctrica específica consumida de película $p P y / D B S$ a diferentes temperaturas, obtenida por integración de los cronopotenciogramas mostrados en la Figura 3.15.

Como una conclusión parcial podemos establecer que la energía consumida por la reacción del material polimérico responde a ( $\mathrm{y}$ por lo tanto siente) las condiciones energéticas (térmicas en este caso) en las que se produce la reacción: a través de la energía consumida el material durante la reacción actúa como un sensor de temperatura.

\section{III.10 Influencia de la concentración en la solución acuosa}

Se siguió la respuesta cronopotenciométrica de las películas poliméricas inmersas en diferentes concentraciones del electrolito para determinar la relación entre la energía 
eléctrica específica consumida y la concentración y estudiar así la posibilidad de que el material pueda ser utilizado como un sensor de la concentración de forma similar a como ocurre con la corriente aplicada o la temperatura. Se empleó un procedimiento experimental similar al utilizado para el estudio de las dos variables previas, siendo 0.09 , $0.15,0.25,0.30,0.40,0.50 \mathrm{M}$ las concentraciones de disolución acuosa $\mathrm{LiClO}_{4}, \mathrm{y}$ aplicando ondas cuadradas de corriente: (a) $+2.25 \mathrm{~mA}$ y (b) $-2.25 \mathrm{~mA}$ durante 40 s cada una de las ramas anódica y catódica, consumiendo cargas constantes de $90 \mathrm{mC}$ a temperatura ambiente

En la Figura 3.17 se muestran las ramas anódicas y catódicas de los cronopotenciogramas estacionarios y normalizados obtenidos entre los mismos estados de oxidación inicial y final del amterial. Se observa una disminución del potencial del material para iguales tiempos de flujo de corriente al aumentar la concentración del electrolito, tanto para la oxidación como la reducción.

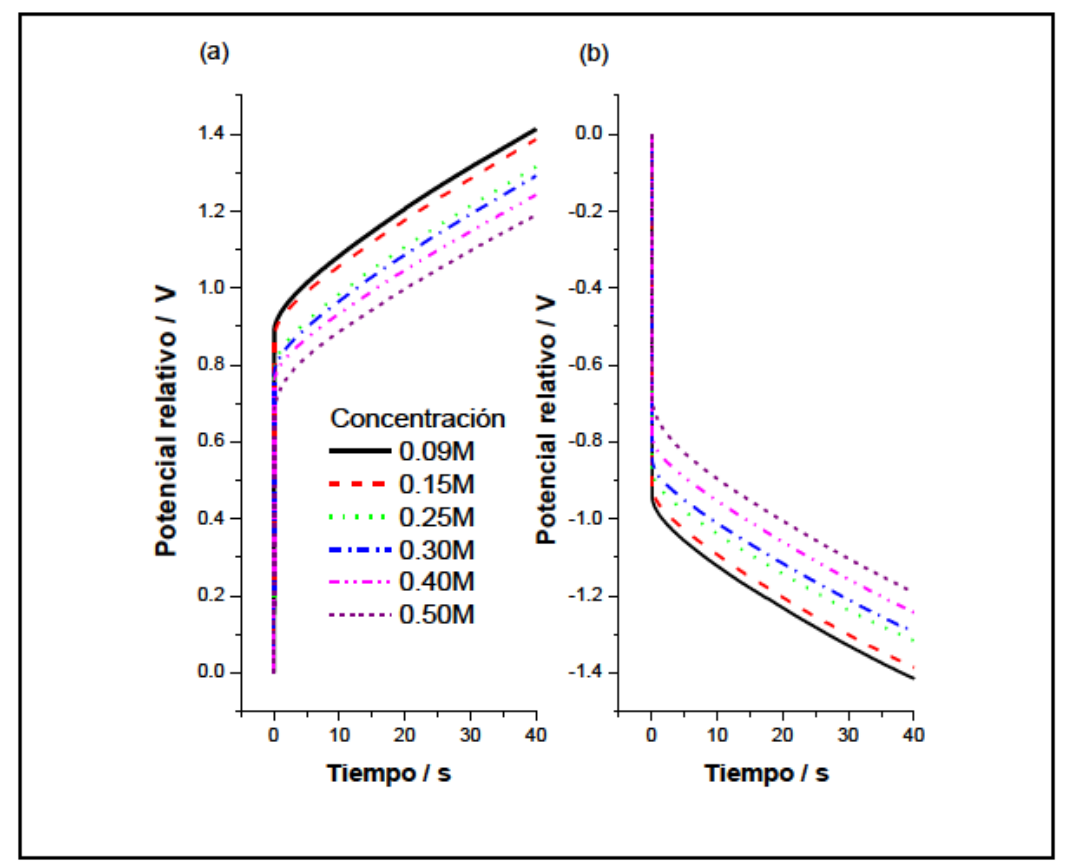

Figura. 3.17 Cronopotenciogramas obtenidos de una película autosoportada pPy/DBS en disoluciones acuosas $\mathrm{LiClO}_{4}$ con diferentes concentraciones de electrolito $(0.09,0.15,0.25,0.30$, $0.40,0.50 \mathrm{M}$ ) aplicando: (a) $+2.25 \mathrm{~mA}$ y (b) $-2.25 \mathrm{~mA}$ durante $40 \mathrm{~s}$ a temperatura ambiente.

Calculada la energía eléctrica específica (por unidad de peso del polímero dentro de la disolución) consumida en cada caso, resultó una función semilogaritmica de la concentración como se observa en la Figura 3.18: la energía consumida por el material estudiado es un sensor semilogarítmico de la concentración de sales en que está trabajando. 


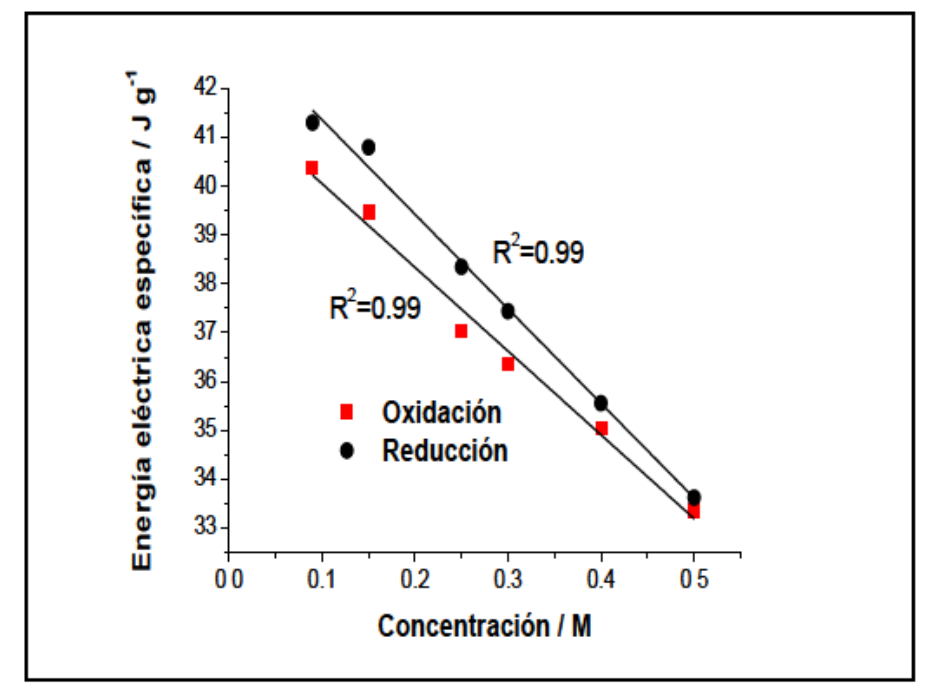

Figura 3.18 Variación de la energía eléctrica consumida durante los cronopotenciogramas mostrados en la figura 3.17 en función de la concentración del electrolito.

Podemos concluir que, tanto la evolución del potential, como la energía consumida por el material para pasar entre los mismos estados de oxidación inicial y final son sensores de las condiciones energéticas (térmicas, químicas o electricas) de trabajo. Como consecuencia cualquier dipositivo construído con el material y que funcione mediante reacción electroquímica del mismo (músculo artificial, batería o supercondensador, ventana electrocrómica, membrana inteligente, interfase computador-neurona, etc.) será, simultáneamente, un actuador y un sensor de las condiciones de trabajo. Las dos señales, actuadora (corriente) y sensora (potencial o energía), estarán incluídas, simultáneamente, en los dos cables de conexión. Similar dualidad actuadora-sensora no tiene paralelo en las tecnologías actuales, sólo la biología ofrece sistemas como los músculos hápticos, origen de la propiocepción mecánica, con tal multifuncionalidad. 


\section{III.11 CARACTERIZACIÓN DE ACTUADORES DE BICAPA}

\section{III.11.1 Introducción}

La literatura presenta numerosos estudios de caracterización de actuadores, músculos artificiales o motores poliméricos de bicapa (polímero conductor//cinta adhesiva) en los que el polímero conductor intercambia aniones con la disolución durante las reacciones de oxidación/reducción que impulsan la actuación ${ }^{5,111,113}$. Aquí se aborda el estudio y se presentan los resultados obtenidos para la caracterización de actuadores poliméricos tipo bicapa pPy/DBS//cinta con intercambio predominantemente de cationes. Las bicapas permiten transformar las pequeñas variaciones reversibles de volumen de una película de polímero conductor impulsados por las reacciones electroquímicas en movimientos macroscópicos de flexión cuando se pasa corriente eléctrica (promoviendo así la reacción (1.2) a través del dispositivo $26,108,109,155,197$. Asimismo se pretende comprobar si el origen electroquímico del movimiento de dichas bicapas pPy/DBS es descrito por las ecuaciones lineales propias de sistemas farádicos, tal y como ocurre con bicapas basadas en polímeros conductores con intercambio de aniones ${ }^{108,152}$.

El procedimiento experimental para el estudio de actuadores bicapa pPy/DBS//cinta es análogo al descrito anteriormente para el estudio del material (películas autosoportadas). Los experimentos se inician siempre aplicando voltamperometría cíclica al polímero conductor de la bicapa para obtener su respuesta voltamperométrica, que nos servirá como control del estado del dispositivo y nos asegurará la reproducibilidad de los resultados en cuanto a su electroactividad. Se ha de asegurar, además, la reproducibilidad actuadora: cualquier actuador con las mismas propiedades y estructura y formado por un material con la misma electroactividad debe describir un ángulo similar (en su extremo libre).

\section{III.11.2 Caracterización del movimiento de los músculos poliméricos tipo bicapa pPy/DBS.}

En este apartado se hace una descripción cuantitativa del movimiento de los actuadores bicapa. La Figura 3.19a muestra los movimientos de flexión de la bicapa (la cinta adhesiva en el lado derecho y la película pPy/DBS en el lado izquierdo) para describir movimientos hasta $\pm 90^{\circ}$ desde la posición vertical (Figura.3.19b). Se observa que bajo el flujo de corrientes anódicas la película de pPy/DBS se contrae por la oxidación tirando de la bicapa (movimiento angular siguiendo las manecillas del reloj). Bajo el flujo de una corriente catódica, el pPy/DBS se expande empujando la bicapa 
(el movimiento angular descrito en sentido contrario a las manecillas del reloj) por la reducción en disolución $0.1 \mathrm{M} \mathrm{LiClO}_{4}$ (Figura 3.19c).

Las películas de polipirrol reportadas en la literatura que trabajan con intercambio de aniones durante las reacciones presentan cambios de volumen y movimientos opuestos: se expanden durante la oxidación y se contraen durante la reducción ${ }^{113} \mathrm{De}$ esta forma el movimiento de la bicapa corrobora que el cambio de volumen del polímero conductor es debido al intercambio de cationes en todo el rango de potenciales estudiado, tal y como lo establece la reacción (1.2). El DBS es un contraión grande, por lo que permanece atrapado en la película polimérica (reacción 1.2). Por ello, la electroneutralidad en la película se mantendrá con intercambio de cationes de $\mathrm{Li}^{+}$desde la disolución acuosa de $\mathrm{LiClO}_{4}$, en los procesos de oxidación reducción.
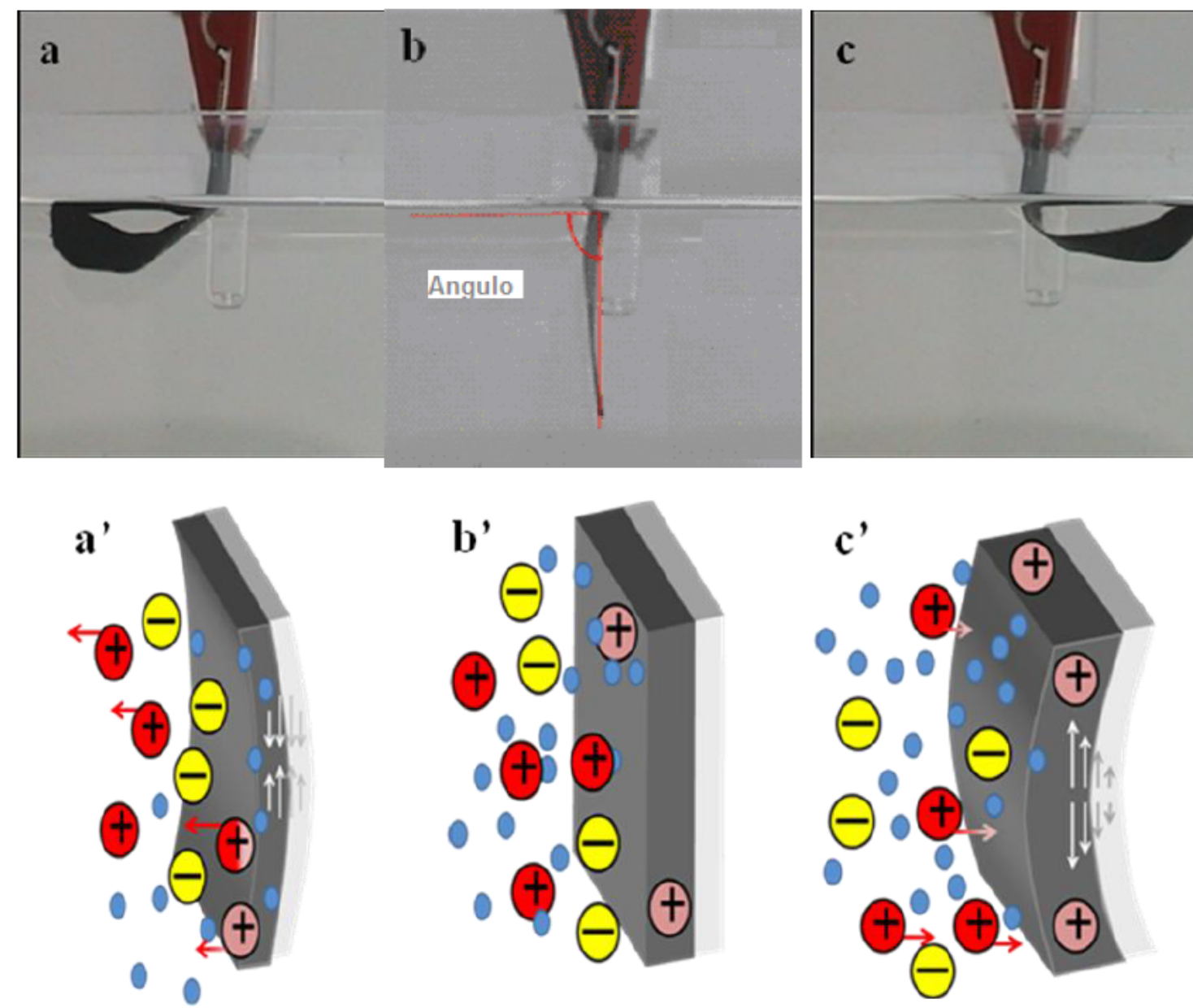

Figura 3.19. Movimientos de combamiento de un actuador pPy/DBS//cinta: a, a') flujo de corriente anódica $\left.b, b^{\prime}\right)$ Representación de la distribución de iones en el músculo $c, c^{\prime}$ ) flujo de corriente catódica 


\section{III.11.3 Caracterización del movimiento (velocidad angular/ corriente) del músculo artificial pPy/DBS//cinta}

Al caracterizar el movimiento y las propiedades de los actuadores, se comprobó si, al igual que en el caso de actuadores basados en polímeros conductores con intercambio aniónico ${ }^{108,152}$, el comportamiento del actuador polimérico es farádico. De acuerdo con la reacción (1.2), la composición del material será función del estado de oxidación/reducción del material, que puede ser controlado mediante la carga consumida (electrones extraídos/insertados de/en las cadenas poliméricas). Por ello, la variación de volumen en el pPy/DBS, y por tanto el ángulo descrito por la bicapa, debería de estar controlado por la carga consumida durante dicha reacción; y la velocidad del movimiento, por la velocidad de intercambio de dichos iones (corriente eléctrica aplicada). Para ver la relación entre carga y movimiento se ha estudiado el tiempo requerido por el actuador pPy/DBS//cinta para describir un ángulo constante hacia la derecha o izquierda de $\pm 30^{\circ}$ ( $\pi / 6$ radianes) a partir de la posición vertical, bajo el flujo de una corriente constante distinta cada vez, en el intervalo de $\pm 1 \mathrm{~mA}$ a $\pm 15 \mathrm{~mA}$. Simultáneamente al paso de corriente se registró la respuesta cronopotenciométrica del dispositivo durante el paso de corrientes anódicas y catódicas.

La velocidad angular $(\omega)$ del músculo, se determinó con el apoyo del sistema audiovisual a partir de los fotogramas del video grabado durante el movimiento (Apartado II.8 del capítulo II). El resultado se representa en la Figura 3.20 a), observándose que la velocidad angular del movimiento originado por las reacciones electroquímicas es una función lineal de la corriente aplicada (I). La velocidad angular ( $\omega)$ del músculo es descrita por la ecuación (3.1):

$$
\omega(\mathrm{rad} / \mathrm{s})=k \cdot i(\mathrm{rad} / \mathrm{s} \mathrm{A})(\mathrm{A})
$$

donde $\mathrm{k}$ es el ángulo descrito por unidad de tiempo y por unidad de flujo de corriente $[\mathrm{rad} /(\mathrm{s} \mathrm{A})]$. Dicha $\mathrm{k}$ es una magnitud característica (constante amperodinámica) del sistema polímero conductor-adhesivo-electrolito.

Esta ecuación establece que el actuador electroquímico es un motor eléctrico, es decir, la intensidad de la corriente ( $I$ ) controla la velocidad de movimiento angular y, por lo tanto, el movimiento aumenta su velocidad al aumentar la corriente y disminuye al reducir la corriente, con una pendiente de $3.49 \times 10^{-3}$ ( $\left.\mathrm{rad} / \mathrm{s}\right) / \mathrm{A}$ para los actuadores estudiados. 


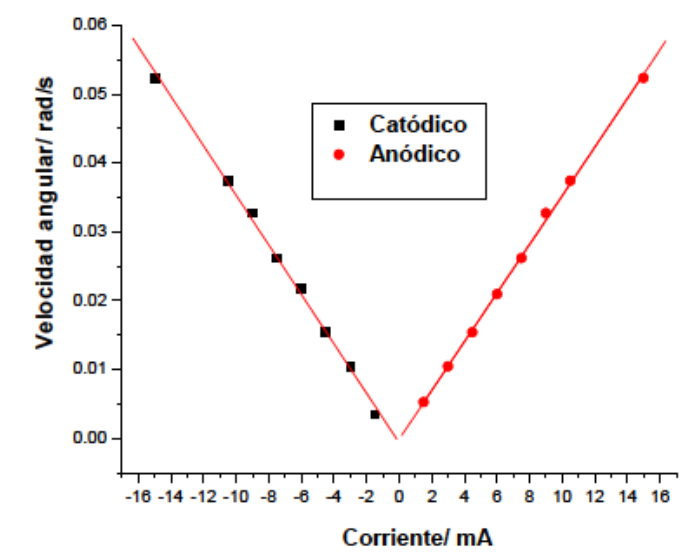

a)

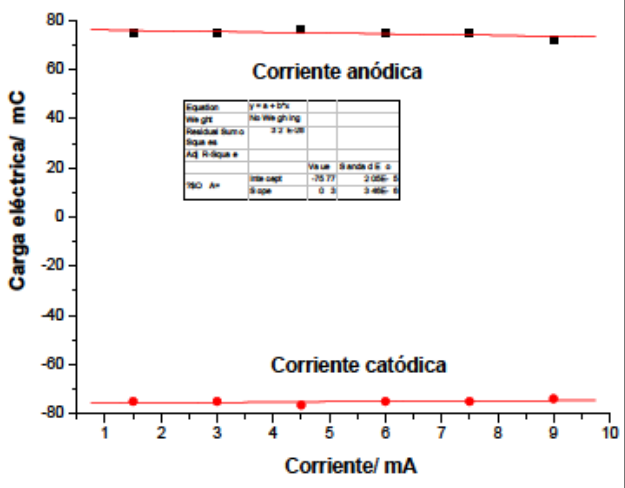

b)

Figura 3.20 a) Relación lineal entre la corriente aplicada y la velocidad angular obtenida para un movimiento de $\pm 30^{\circ}$ de un músculo pPy/DBS//cinta en una disolución acuosa $0.1 \mathrm{M} \mathrm{LiClO}_{4}$ a temperatura ambiente aplicando diferentes corrientes eléctricas b) carga eléctrica consumida por el músculo para realizar los movimientos descritos para (a).

Por otro lado, en la Figura 3.20 b se observa cómo la carga eléctrica consumida para alcanzar los $\pm 30^{\circ}$ es constante cualquiera que sea la corriente aplicada: el desplazamiento no depende de la corriente aplicada, sino de la carga consumida, al igual que ocurría con los actuadores basados en polímeros con intercambio aniónico $108,152,163$

$$
\alpha(\mathrm{rad})=\omega(\mathrm{rad} / \mathrm{s}) \cdot t(\mathrm{~s})=k(\mathrm{rad} /(\mathrm{s} \mathrm{A})) \cdot \mathrm{l}(\mathrm{A}) \cdot \mathrm{t}(\mathrm{s})=k(\mathrm{rad} /(\mathrm{s} \mathrm{A})) \cdot \mathrm{Q}(\mathrm{C})
$$

Donde: $Q=i t$, es la carga $(C)$ que se consume. La ecuación 3.2 indica que el ángulo descrito por el movimiento angular está bajo control de la carga, $Q$, consumida por el flujo de una corriente constante $i$ durante un tiempo $t$.

A partir de la ecuación 3.2 se deduce que la carga $(Q)$ necesaria para que el actuador alcanze cualquier nueva posición (ángulo) será:

$$
Q(C)=k^{\prime}(C / r a d) \cdot \alpha(\mathrm{rad})
$$

Donde k' ( $\mathrm{C} / \mathrm{rad}$ ) es la constante (culodinámica) característica del sistema que representa la carga consumida para describir un ángulo constante de un radián. Sin importar la posición inicial del extremo libre del músculo (bicapa), la carga necesaria para producir un movimiento angular de un radián debe ser constante, y es una magnitud característica del sistema musculo-electrolito. 
Con los resultados obtenidos se concluye que los parámetros de ingeniería para movimientos macroscópicos de: flexión, dirección de movimiento, ángulos y posiciones finales de los motores electroquímicos, están bajo el control de la dirección del flujo de corriente y la carga consumida, lo que significa que están bajo control farádico de la composición del polímero conductor, como se ha encontrado para músculos bicapa y tricapa de películas de $\mathrm{pPy} / \mathrm{ClO}_{4}{ }^{5,141,215,216}$.

\section{III.11.4 Intercambio de cationes y agua durante la actuación.}

En el apartado anterior se ha comprobado que la reacción electroquímica (1.2) controla el movimiento del músculo artificial. Durante la reducción el polímero se hincha y las cadenas de polímero cambian sus conformaciones generando el volumen libre necesario para acomodar a los cationes necesarios para mantener la electroneutralidad y moléculas de agua requeridas para mantener el equilibrio osmótico. Por tanto el intercambio de agua será otra magnitud a determinar para una mejor comprensión del mecanismo de actuación de estos actuadores.

Para ello se utilizó la bicapa pPy/DBS//cinta, estudiando su movimiento, de forma similar a la descrita en el apartado anterior, en diferentes electrolitos (las sales utilizadas fueron $\mathrm{LiCl}, \mathrm{NaCl}, \mathrm{MgCl}^{2}, \mathrm{CaCl}^{2}, \mathrm{KCl}, \mathrm{RbCl}, \mathrm{CsCl}$ y $\mathrm{LaCl}^{3}$ ).

Tabla 3.1. Cargas específicas de oxidación y reducción de los voltamperogramas de control realizados para los diferentes músculos en el electrolito de referencia (disolución acuosa $0.1 \mathrm{M}$ $\mathrm{NaCl}$ ) entre -1 y $0.2 \mathrm{~V}$ frente a $\mathrm{Ag} / \mathrm{AgCl}$ a $6 \mathrm{mV} / \mathrm{s}$, antes (pre-control) y después (post-control) de los experimentos en los diferentes electrolitos.

\begin{tabular}{|cccccc|}
\hline Sal & $\begin{array}{c}\text { Masa } \\
\text { electroactiva } \\
{[\mathrm{mg}]}\end{array}$ & $\begin{array}{c}\text { Carga de } \\
\text { Oxidación } \\
\text { específica, pre- } \\
\text { control } \\
{[\mathrm{C} / \mathrm{g}]}\end{array}$ & $\begin{array}{c}\text { Carga de } \\
\text { Reducción } \\
\text { especifica, pre- } \\
\text { control } \\
{[\mathrm{C} / \mathrm{g}]}\end{array}$ & $\begin{array}{c}\text { Carga de } \\
\text { Oxidación } \\
\text { especifica post- } \\
\text { control } \\
{[\mathrm{C} / \mathrm{g}]}\end{array}$ & $\begin{array}{c}\text { Carga de Reducción } \\
\text { especifica, post-control } \\
{[\mathrm{C} / \mathrm{g}]}\end{array}$ \\
$\mathrm{LiCl}$ & 1.34 & 91 & -97 & 96 & -103 \\
$\mathrm{NaCl}$ & 1.34 & 78 & -88 & 80 & -92 \\
$\mathrm{MgCl}_{2}$ & 1.30 & 113 & -115 & 113 & -119 \\
$\mathrm{CaCl}_{2}$ & 1.34 & 84 & -89 & 87 & -89 \\
$\mathrm{KCl}$ & 1.34 & 64 & -69 & 66 & -69 \\
$\mathrm{RbCl}$ & 1.15 & 65 & -69 & 71 & -76 \\
$\mathrm{CsCl}$ & 1.96 & 73 & -77 & 77 & -79 \\
$\mathrm{LaCl}_{3}$ & 1.38 & 64 & -76 & 64 & -77 \\
\hline
\end{tabular}

Para asegurar la reproducibilidad de los diferentes actuadores utilizados en los experimentos, se controlaron mediante voltamperometría las cargas específicas para cada actuador a lo largo de las series experimentales. Después de cada serie el 
estado del músculo se comprobó mediante un ciclado de control, comparando la respuesta voltamperométrica en la disolución de referencia $(0.1 \mathrm{M} \mathrm{NaCl})$. La carga específica se determinó teniendo en cuenta la carga reversible del voltamperograma y la masa de pPy/DBS de la bicapa electroactiva dentro de la disolución.

Cuando se encontró una diferencia de carga entre los controles inicial y final mayor al $10 \%$, se utilizó una nueva bicapa con una carga específica similar asegurando así la reproducibilidad, Tabla 3.1. Un ejemplo de controles iniciales y finales tras el estudio de dos sales diferentes $\left(\mathrm{RbCl}\right.$ y $\left.\mathrm{MgCl}_{2}\right)$ se presenta en la figura 3.21. Además de la reproducibilidad de la electroactividad, se aseguró la reproducibilidad del movimiento observando la amplitud del movimiento durante dichos controles. Comparando las cargas específicas anódicas aplicadas a los músculos en el electrolito de referencia $\mathrm{NaCl}$ (Tabla 3.2) cada $\mathrm{mC}$ de carga aplicada por gramo de polipirrol activo en el músculo promueve un ángulo descrito similar $(0.342 \pm 0.043) \% \mathrm{Cg})$. El movimiento del actuador muestra una buena reproducibilidad en ambas direcciones con los distintos actuadores utilizados.

Se realizaron también experimentos cronopotenciométricos. Los cronopotenciogramas siempre se inician desde el mismo potencial después de la pre-polarización inicial, siguiendo el procedimiento experimental mostrado en la Figura 2.5.

a)

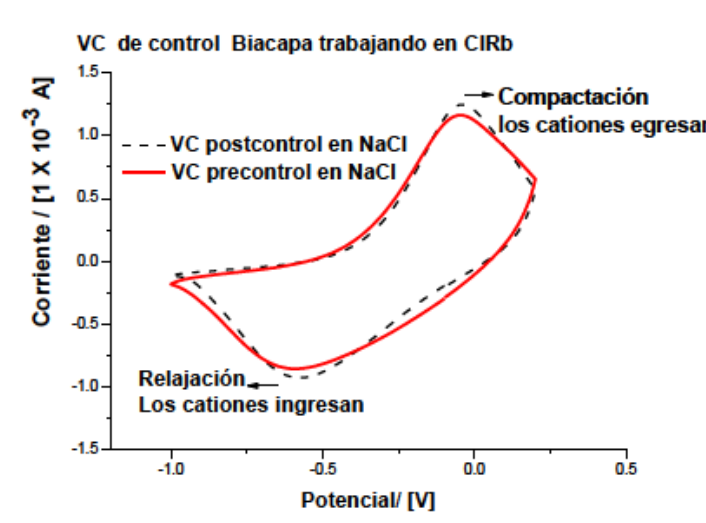

b)

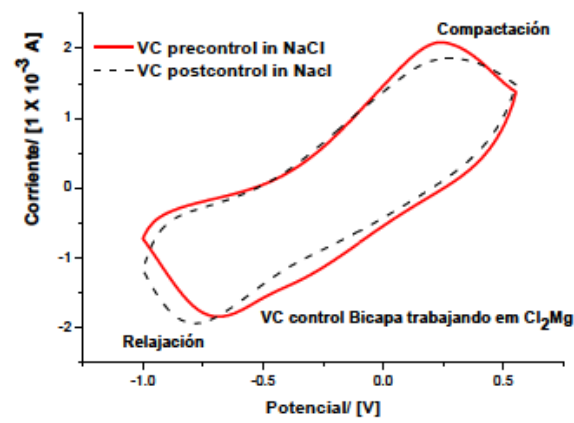

Figura 3.21. Voltamperogramas de control obtenidos en disolución acuosa $0.1 \mathrm{M}$ de $\mathrm{NaCl}$ entre $-1 \mathrm{~V}$ y $0.2 \mathrm{mV}$, a $6 \mathrm{mV} / \mathrm{s}$ a temperatura ambiente con un desplazamiento del músculo de $50^{\circ}$ después de: (a) experimentos galvanostáticas en cloruro de rubidio $(\mathrm{RbCl})$ y (b) experimentos galvanostáticas en sal de cloruro de magnesio $\mathrm{MgCl}_{2}$

Una vez asegurada la reproducibilidad del movimiento, se obtuvieron los resultados experimentales en diferentes electrolitos mediante la aplicación de ondas de cuadradas de corriente utilizando diferentes corrientes constantes, $( \pm 0.40, \pm 0.60, \pm$ 
0.80, $\pm 1.00, \pm 1.40, \pm 1.80, \pm 2.00, \pm 2.25, \pm 2.5, \pm 2.75, \pm 3 \mathrm{~mA})$, manteniendo constantes el resto de variables experimentales $(80 \mathrm{mC}$ de carga anódica o catódica, temperatura ambiente y concentración de electrolito $0.1 \mathrm{M}$ ).

Tabla 3.2. Oxidación y reducción de los picos obtenidos a partir de las respuestas pre-control y post-control voltamperométricas de las bicapas pPy/DBS//cinta en $0.1 \mathrm{M}$ disolución acuosa de $\mathrm{NaCl}$ entre - 1 y $0.2 \mathrm{~V}$ frente a $\mathrm{Ag} / \mathrm{AgCl}$ en $6 \mathrm{mV} / \mathrm{s}$.

\begin{tabular}{|c|c|c|c|c|c|}
\hline Electrolito & $\begin{array}{l}\text { Angulo por carga } \\
\text { especifica en } \\
\mathrm{NaCl} \\
{\left[{ }^{\circ}(\mathrm{C} / \mathrm{g})\right]}\end{array}$ & $\begin{array}{l}\text { Pico de } \\
\text { oxidación pre- } \\
\text { control [mV] }\end{array}$ & $\begin{array}{l}\text { Pico de } \\
\text { reducción pre- } \\
\text { control [mV] }\end{array}$ & $\begin{array}{l}\text { Pico de } \\
\text { Oxidación post- } \\
\text { control }[\mathrm{mV}]\end{array}$ & $\begin{array}{l}\text { Pico de } \\
\text { Reducción pico } \\
\text { post-control } \\
{[\mathrm{mV}]}\end{array}$ \\
\hline $\mathrm{LiCl}$ & 0.35 & -0.12 & -0.60 & -0.14 & -0.70 \\
\hline $\mathrm{NaCl}$ & 0.34 & -0.05 & -0.57 & -0.022 & -0.58 \\
\hline $\mathrm{MgCl}_{2}$ & 0.35 & 0.13 & -0.10 & 0.17 & -0.08 \\
\hline $\mathrm{CaCl}_{2}$ & 0.35 & 0.05 & -0.65 & 0.05 & -0.70 \\
\hline $\mathrm{KCl}$ & 0.34 & -0.05 & -0.55 & -0.05 & -0.55 \\
\hline $\mathrm{RbCl}$ & 0.38 & -0.12 & -0.58 & -0.09 & -0.61 \\
\hline $\mathrm{CsCl}$ & 0.38 & 0.33 & -0.72 & 0.11 & -0.58 \\
\hline $\mathrm{LaCl}_{3}$ & 0.36 & -0.07 & -0.58 & -0.06 & -0.65 \\
\hline
\end{tabular}

La evolución del potencial del músculo respecto al electrodo de referencia ocurre a potenciales tanto más altos cuanto mayores son corrientes aplicadas en los diferentes electrolitos estudiados (Figura 3.22), de forma similar a como ocurría con las películas autosoportadas (Apartado 3.8). Se observa un salto del potencial muscular al inicio del pulso de corriente, que estará relacionado con las diferentes resistencias del sistema electroquímico hasta iniciar la reacción del polímero conductor. Una vez iniciada la reacción el potencial aumenta (más rápido cuanto mayor es la corriente aplicada) al aumentar con el tiempo de flujo de corriente debido a la creciente resistencia de la reacción (ecuación electroquímica 1.2) cuando la concentración de los centros activos (reactivos) en la película disminuye. 

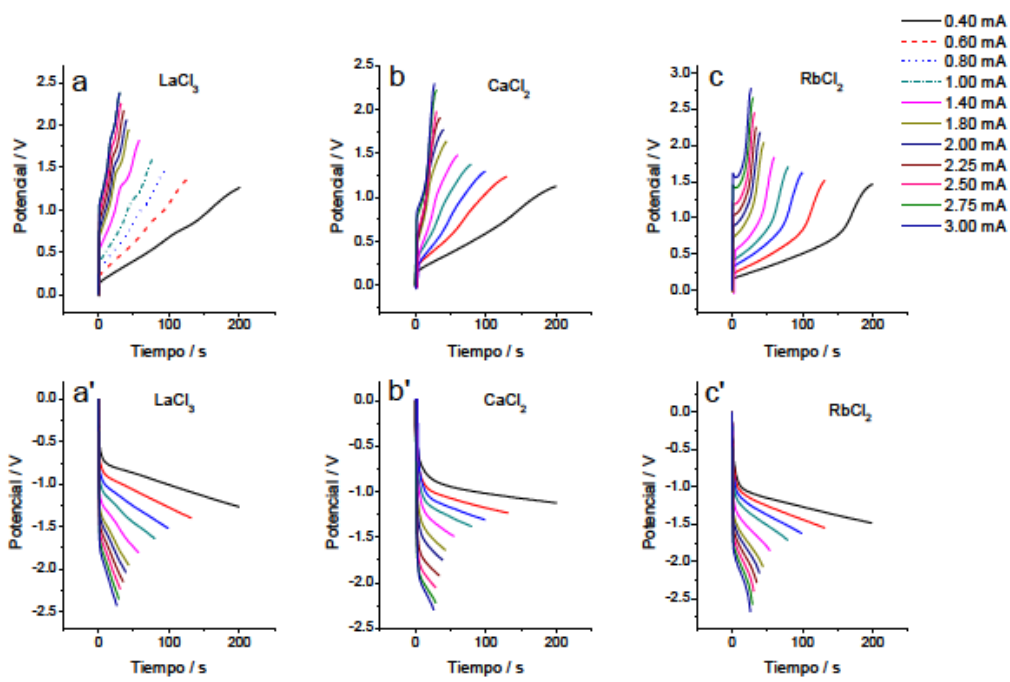

Figura 3.22. Respuestas cronopotenciométricas obtenidas al aplicar diferentes corrientes constantes (indicadas en la Figura) de una bicapa pPy/DBS//cinta en diferentes electrolitos acuosos $0.1 \mathrm{M}$ de las diferentes sales indicadas en la Figura a $25^{\circ} \mathrm{C}$. (a) las respuestas anódicos en $\mathrm{LaCl}_{3}$. (a ') respuestas catódica en $\mathrm{LaCl}_{3}$; (b) las respuestas anódicos en $\mathrm{CaCl}_{2}$; (b ') respuestas catódica en $\mathrm{CaCl}_{2}$, (c) respuesta anódica de $\mathrm{RbCl}$; (c ') la respuesta catódica de $\mathrm{RbCl}$.

El movimiento de las bicapas pPy/DBS//cinta durante el paso de corriente en diferentes electrolitos se registró mediante vídeo. A partir de los fotogramas del vídeo, los ángulos descritos para diferentes tiempos de flujo de corriente (después de consumir diferentes cargas específicas) en cada uno de los electrolitos estudiados fueron determinados. Los resultados culodinámicos (carga-ángulo) se presentan en la Figura 3.23 para los distintos electrolitos.

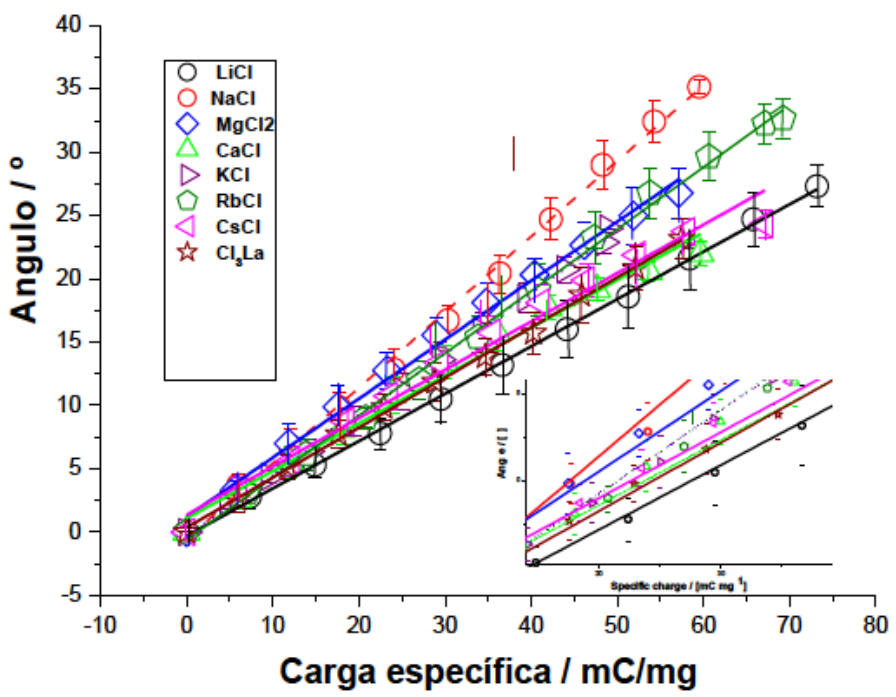

Figura 3.23. Evolución de los ángulos descritos por una bicapa pPy/DBS//cinta en función de la carga específica consumida bajo el flujo de diferentes corrientes constantes (de \pm 0.4 a $\pm 3 \mathrm{~mA}$ ) para diferentes tiempos (se obtuvieron resultados para 11 diferentes corrientes aplicadas), en disoluciones acuosas $0.1 \mathrm{M}$ de las sales estudiadas. 
Se obtuvieron relaciones lineales entre la carga específica aplicada $\left(\mathrm{mC} \mathrm{mg}^{-1}\right)$ y el desplazamiento angular confirmando los resultados obtenidos en el apartado III.11.3. Estos resultados corroboran el control farádico del movimiento impulsado por la reacción (1.2) con una pendiente distinta para cada una de las sales. La Figura 3.23 corrobora que la velocidad de los cambios de volumen en la película de pPy/DBS (y en los ángulos descritos por la bicapa pPy/DBS//cinta) se producen bajo control (dependencia lineal) de la carga específica aplicada por unidad de tiempo (corriente eléctrica aplicada) para músculos artificiales con intercambio de cationes en diferentes electrolitos.

También se confirma que los sentidos del movimiento impulsado por las corrientes es el mismo en los diferentes electrolitos lo que indica que sólo se intercambian cationes, siguiendo la reacción (1.2). El inicio de un intercambio paralelo de cationes y aniones en cualquier momento de la reacción provocaría un cambio de la pendiente. La presencia de un máximo indicaría que el intercambio de aniones comienza a prevalecer durante la reacción. Como conclusión parcial la Figura 3.23 corrobora que los cationes son los únicos iones intercambiados durante la actuación en todos los electrolitos estudiados.

De acuerdo con la reacción (1.2), las variaciones de volumen película pPy/DBS son debidas al intercambio de cationes y al del disolvente necesario para mantener el equilibrio osmótico en el interior del gel polimérico. Por ello, la variación de volumen inducida por la reacción (1.2) en la película pPy/DBS se puede expresar como:

$$
\Delta V=\Delta V_{\text {iones }}+\Delta V_{\text {solvente }}
$$

Donde $\Delta \mathrm{V}$ se refiere a la variación de volumen pPy/DBS, $\Delta \mathrm{V}_{\text {iones }}$ es la variación de volumen debido al intercambio de cationes y $\Delta \mathrm{V}_{\text {solvente }}$ es la variación de volumen debido al intercambio de moléculas de disolvente durante la reacción 1.2.

El incremento de volumen durante la reducción $\Delta \mathrm{V}_{\text {red }}$ debido al número de cationes intercambiados, que es definido por la ley de Faraday a partir de la carga consumida durante la reducción $\left(\mathrm{Q}_{\text {red }}\right)$ multiplicado por el volumen cristalográfico del catión, calculado a partir del radio cristalográfico $r$, considerando que los iones esféricos.

$$
\Delta V_{\text {red }}=\frac{Q_{\text {red }}}{z F} N_{A} \frac{4}{3} \pi r^{3}
$$


Donde: $\left(Q_{\mathrm{red}} / \mathrm{zF}\right) \mathrm{N}_{\mathrm{A}}$ es el número de cationes intercambiados, siendo $\mathrm{z}$ la carga del ión, $\mathrm{F}$ la constante de Faraday $(\mathrm{F}=96485 \mathrm{C} / \mathrm{mol}), \mathrm{N}_{\mathrm{A}}$ el número de Avogadro, y (4/3 $\pi r^{3}$ ) es el volumen del catión, siendo r el radio cristalográfico del catión ${ }^{217}$. Los cationes de los electrolitos aquí estudiados tienen diferentes cargas, diferentes radios cristalográficos ${ }^{218}$, y diferentes volúmenes iónicos, como se muestra en la tabla 3.3.

\begin{tabular}{|c|c|c|c|c|c|}
\hline Sal & $\begin{array}{l}\text { Masa activa pPy / } \\
\text { mg }\end{array}$ & Radio Cationes / nm & Volumén Cation/ $\mathrm{nm}^{3}$ & Angúlo/ ${ }^{\circ}$ & Pendiente $\mathrm{k} / \mathrm{rad} / \mathrm{C} \mathrm{g}$ \\
\hline $\mathrm{LiCl}$ & 1.07 & 0.07 & $1.15 \times 10^{-3}$ & 14.67 & 0.39 \\
\hline $\mathrm{NaCl}$ & 1.35 & 0.10 & $4.10 \times 10^{-3}$ & 23.35 & 0.60 \\
\hline $\mathrm{MgCl}_{2}$ & 1.30 & 0.06 & $0.80 \times 10^{-3}$ & 18.83 & 0.47 \\
\hline $\mathrm{CaCl}_{2}$ & 1.34 & 0.10 & $4.20 \times 10^{-3}$ & 15.14 & 0.38 \\
\hline $\mathrm{KCl}$ & 1.34 & 0.14 & $10.80 \times 10^{-3}$ & 18.87 & 0.48 \\
\hline $\mathrm{RbCl}$ & 1.15 & 0.15 & $14.70 \times 10^{-3}$ & 19.02 & 0.49 \\
\hline $\mathrm{CsCl}$ & 1.38 & 0.17 & $19.50 \times 10^{-3}$ & 16.64 & 0.38 \\
\hline $\mathrm{LaCl}_{3}$ & 1.99 & 0.11 & $4.70 \times 10^{-3}$ & 16.17 & 0.39 \\
\hline
\end{tabular}

De la ecuación 3.5, y teniendo en cuenta las relaciones de la ecuación 3.3, la variación del volumen de la película pPy/DBS y el ángulo de desplazamiento para una carga constante, si dependieran sólo de los cationes intercambiados, deberían ser una función cúbica del radio iónico:

$$
\alpha=k q=k \frac{3}{4} \frac{F_{z} \Delta V_{\text {red }}}{N_{A} \pi r^{3}} \Leftrightarrow \Delta V_{\text {red }}=\frac{4 N_{A} \pi r^{3}}{3 k F z} \alpha
$$

La Ecuación 3.6 correlaciona la constante del sistema (k), la evolución del volumen pPy/DBS $\left(\Delta \mathrm{V}_{\text {red }}\right)$, el ángulo descrito $(\alpha)$, el radio cristalográfico $(r)$ y el número de cargas por catión $(z)$ en una primera aproximación (sin considerar el intercambio de disolvente). En estas condiciones, el electrolito en que se obtuvo la amplitud de movimiento más baja del movimiento por carga específica fue el que contenía la sal de $\mathrm{Cs}^{+}$(Tabla 3.4).

La Figura 3.23 muestra diferentes pendientes para cada uno de los electrolitos estudiados. Para una carga específica constante de, por ejemplo, $40 \mathrm{mC} / \mathrm{mg}$ la amplitud del desplazamiento angular cambia con el catión intercambiado siguiendo la secuencia: $\mathrm{Na}^{+}>\mathrm{Rb}^{+}>\mathrm{K}^{+}>\mathrm{Mg}^{2+}>\mathrm{Cs}^{+}>\mathrm{La}^{3+}>\mathrm{Ca}^{2+}>\mathrm{Li}^{+}$(Tabla 3.3). 
Esta secuencia experimental no sigue los radios cristalográficos de los cationes de la

Tabla 3.3: $\mathrm{Cs}^{+}>\mathrm{Rb}^{+}>\mathrm{K}^{+}>\mathrm{La}^{2+}>\mathrm{Ca}^{2+}>\mathrm{Na}^{+}>\mathrm{Li}^{+}>\mathrm{Mg}^{2+}$.

Tomando las magnitudes relacionadas con $\mathrm{CsCl}$ como referencia, podemos determinar los desplazamientos angulares relativos por el flujo de una carga específica constante, $\alpha($ cation $) / \alpha\left(\mathrm{Cs}^{+}\right)$; el radio cristalográfico relativo, $r$ (catión) $/ r\left(\mathrm{Cs}^{+}\right), y$ el volumen iónico relativo por unidad de carga, $\mathrm{v}$ (cation)/z v(Cs$\left.{ }^{+}\right)$siendo $\mathrm{z}$, el número de cargas por catión.

\begin{tabular}{|c|c|c|c|c|c|c|c|c|}
\hline Catión & $\mathrm{Li}^{+}$ & $\mathrm{Na}^{+}$ & $\mathrm{Mg}^{2+}$ & $\mathrm{Ca}^{+}$ & $\mathrm{K}^{+}$ & $\mathrm{Rb}^{+}$ & $\mathrm{Cs}^{+}$ & $\mathrm{La}^{3+}$ \\
\hline$\alpha($ Cat. $) / \alpha(C s)$ & 0.88 & 1.40 & 1.131 & 0.91 & 1.13 & 1.14 & 1.00 & 0.97 \\
\hline$r($ Cat. $) / r(C s)$ & 0.39 & 0.59 & 0.34 & 0.60 & 0.82 & 0.91 & 1.00 & 0.62 \\
\hline $\mathrm{V}($ Cat. $) N(\mathrm{Cs})$ & 0.06 & 0.21 & 0.079 & 0.43 & 0.55 & 0.75 & 1.00 & 0.70 \\
\hline
\end{tabular}

La Tabla 3.4 muestra que el mayor desplazamiento relativo del músculo se obtiene en disoluciones acuosas de $\mathrm{Na}^{+}$, seguido de las soluciones de: $\mathrm{Rb}^{+}, \mathrm{Mg}^{2+}, \mathrm{K}^{+}, \mathrm{La}^{3+}, \mathrm{Ca}^{2+}$, $\mathrm{Li}^{+}$. Sin embargo, el mayor volumen iónico relativo corresponde a $\mathrm{Rb}^{+}$seguido de $\mathrm{La}^{3+}$, $\mathrm{K}^{+}, \mathrm{Ca}^{2+}, \mathrm{Na}^{+}, \mathrm{Mg}^{2+}, \mathrm{Li}^{+}$. Como conclusión parcial, a pesar del origen farádico (Figura 3.23) del movimiento, el intercambio de cationes no puede justificar, por si solo, la variación de volumen de la película pPy/DBS y la amplitud del movimiento descrito.

\begin{tabular}{|c|c|c|c|}
\hline Electrolito & Moléculas de agua/ catión & Moléculas de agua / carga & $\begin{array}{l}\text { Composición de las películas d } \\
\text { reducidas }\end{array}$ \\
\hline $\mathrm{LiCl}$ & 0.535 & 0.535 & $(\mathrm{PPy} / \mathrm{DBS})^{n-}{ }_{s}\left(\mathrm{Li}^{+}\right)_{n} \mathrm{H} 2 \mathrm{O} 0_{.5 \mathrm{n}}$ \\
\hline $\mathrm{NaCl}$ & 0.777 & 0.777 & $(\mathrm{PPy} / \mathrm{DBS})^{n^{-}}\left(\mathrm{Na}^{+}\right)_{n} \mathrm{H} 2 \mathrm{O} 0_{.7 n}$ \\
\hline $\mathrm{MgCl}_{2}$ & 0.723 & 0.361 & $(\mathrm{PPy} / \mathrm{DBS})^{\mathrm{n} 2-}{ }_{s}\left(\mathrm{Mg}^{2+}\right)_{\mathrm{n}} \mathrm{H} 2 \mathrm{O} 0_{35 n}$ \\
\hline $\mathrm{CaCl}_{2}$ & 0.521 & 0.252 & $(\text { PPy/DBS })^{\mathrm{n} 2-}{ }_{s}\left(\mathrm{Ca}^{2+}\right)_{n} \mathrm{H} 2 \mathrm{O} 0_{-25 \mathrm{n}}$ \\
\hline $\mathrm{KCl}$ & 0.378 & 0.378 & $(\mathrm{PPy} / \mathrm{DBS})_{s}^{n_{s}}\left(\mathrm{~K}^{+}\right)_{n} \mathrm{H} 2 \mathrm{O} 0_{-4 n}$ \\
\hline $\mathrm{RbCl}$ & 0.252 & 0.252 & $(\mathrm{PPy} / \mathrm{DBS})^{n^{-}}{ }_{s}\left(\mathrm{Rb}^{+}\right)_{n} \mathrm{H} 2 \mathrm{O} 0_{25 n}$ \\
\hline $\mathrm{CsCl}$ & 0.000 & 0.000 & $(\mathrm{PPy} / \mathrm{DBS})^{n-}\left(\mathrm{Cs}^{+}\right)_{n}$ \\
\hline $\mathrm{LaCl}_{3}$ & 0.581 & 0.193 & $(\mathrm{PPy} / \mathrm{DBS})^{\mathrm{n} 3-}{ }_{\mathrm{s}}\left(\mathrm{La}^{3+}\right)_{n} \mathrm{H} 2 \mathrm{O} 0_{2 \mathrm{n}}$ \\
\hline
\end{tabular}

Regresando de nuevo a la reacción (1.2) como origen del movimiento, además del intercambio de cationes ha de considerarse en el cálculo teórico el intercambio de 
moléculas de disolvente durante la reacción. La fracción de la variación de volumen debido al intercambio de agua será el número ( $\mathrm{m}$, desconocido) de moléculas de disolvente intercambiadas multiplicado por el volumen de una molécula de agua $\left(\mathrm{V}_{\mathrm{H} 2 \mathrm{O}}\right)$. La variación de volumen total se puede expresar como:

$$
\Delta V=\Delta V_{\text {red }}+\Delta V_{H_{2} \mathrm{O}}=\frac{4 N_{A} \pi r^{3}}{3 k F z} \alpha+m V_{H_{2} O}
$$

El volumen de $1 \mathrm{~g}$ de agua a $25 \stackrel{\circ}{\circ}$ es $1,0029607 \mathrm{~cm}^{3,218}$ la masa de un mol de agua es de 18 gramos y un mol de agua contiene $6.022 \times 10^{23}$ moléculas, por lo que el volumen de una molécula de agua es $=2,9979 \times 10^{-23} \mathrm{~cm}^{3}$.

La ecuación 3.7 relaciona el aumento de volumen $(\Delta \mathrm{V})$ durante la reducción de la película de pPy/DBS de la bicapa del músculo con el ángulo descrito $(\alpha)$ por el flujo de una carga de reducción constante $\left(Q_{\text {red }}\right)$, el radio cristalográfico $(r)$ del catión, su carga (z) y el número de moléculas de agua intercambiados (m). A partir de esta ecuación y de los resultados experimentales en presencia de distintos cationes es posible obtener el número de moléculas de agua incorporados a la película de pPy/DBS en paralelo a cada catión.

El ángulo descrito en cada uno de los electrolitos estudiados $\left(\alpha_{x}\right)$ se debe a la entrada de los cationes $x$ además de la entrada de $m$ (desconocidos) moléculas de agua $\left(\mathrm{mV}_{\mathrm{H} 2 \mathrm{O}}\right)$. Considerando que el catión que produce el desplazamiento más bajo no intercambia ninguna molécula de agua, los ángulos relativos descritos en diferentes sales para la misma carga específica de la Tabla 3.5 permiten vincular todas esas magnitudes en una simple expresión:

$$
m=\frac{V_{C s} \alpha_{x} / \alpha_{r e f}-V_{x} / z}{V_{H_{2} O}}
$$

Donde: $\mathrm{V}_{\mathrm{Cs}}$ es el volumen cristalográfico del $\left(\mathrm{Cs}^{+}\right), \alpha_{\mathrm{x}} / \alpha_{\text {ref }}$ es el ángulo descrito por el músculo en la sal estudiada relativo al descrito en $\mathrm{CsCl}$ por el flujo de la misma carga específica (Tabla 3.5, primera fila), $V_{x}$ es la volumen cristalográfica del catión $x$ (siendo $\left.\mathrm{x}: \mathrm{Li}^{+}, \mathrm{Na}^{+}, \mathrm{Mg}^{2+}, \mathrm{Ca}^{2+}, \mathrm{K}^{+}, \mathrm{Rb}^{+}, \mathrm{o} \mathrm{La}^{2+}\right)$. Los resultados para las moléculas de agua intercambiadas se muestran en la tabla 3.5.

La tabla 3.5 muestra que alrededor de una molécula de agua se incorpora a la película polimérica desde la disolución al mismo tiempo dos cationes $\mathrm{Li}^{+}, \mathrm{o} \mathrm{Ca}^{2+}, \mathrm{o} \mathrm{La}^{3+}$ durante la reacción de reducción (1.2); Alrededor de dos moléculas de agua se intercambian, en $\mathrm{NaCl} \circ \mathrm{MgCl}_{2}$, cada tres iones $\mathrm{Na}^{+} \circ \mathrm{Mg}^{2+}$; alrededor de una molécula de agua se intercambia en $\mathrm{KCl}$ cada tres iones $\mathrm{K}^{+}$; y alrededor de una molécula de agua se 
intercambia en $\mathrm{RbCl}$ por cuatro iones $\mathrm{Rb}^{+}$. Estos resultados, siempre referenciados a la disolución de $\mathrm{CsCl}$, donde se supone que sólo los cationes se intercambian durante la reacción (o acompañados de un número insignificante de moléculas de disolvente). . Atribuyendo el intercambio de agua sólo a la interacción de cationes-disolvente, el número de solvatación aparente de los cationes debe ser: 0.7, 0.72, 0.58, 0.53, 0.52, 0.37, 0.25 y 0.0 para $\mathrm{Na}^{+}, \mathrm{Mg}^{2+}, \mathrm{La}^{3+}, \mathrm{Li}^{+}, \mathrm{Ca}^{2+}, \mathrm{K}^{+}, \mathrm{Rb}^{+}$and $\mathrm{Cs}^{+}$, respectivamente. Skaarup obtiene números superiores de hidratación de la membrana con electrolitos acuosos de halogenuros alcalinos en películas de polipirrol ${ }^{219,220}$.

La Figura 3.24-a corrobora que a pesar del origen farádico del movimiento (Figura 3.23) no existe ninguna relación lineal entre el volumen iónico de los diferentes cationes y de las pendientes (k) de la Figura 3.23. Teniendo en cuenta ahora el volumen solvatado aparente (entendido como el volumen del catión intercambiado más el volumen del número calculado de las moléculas de agua con cada catión intercambiado) una buena relación lineal se encuentra entre $\mathrm{k}$ y el volumen aparente solvatado (Figura 3.24 b).

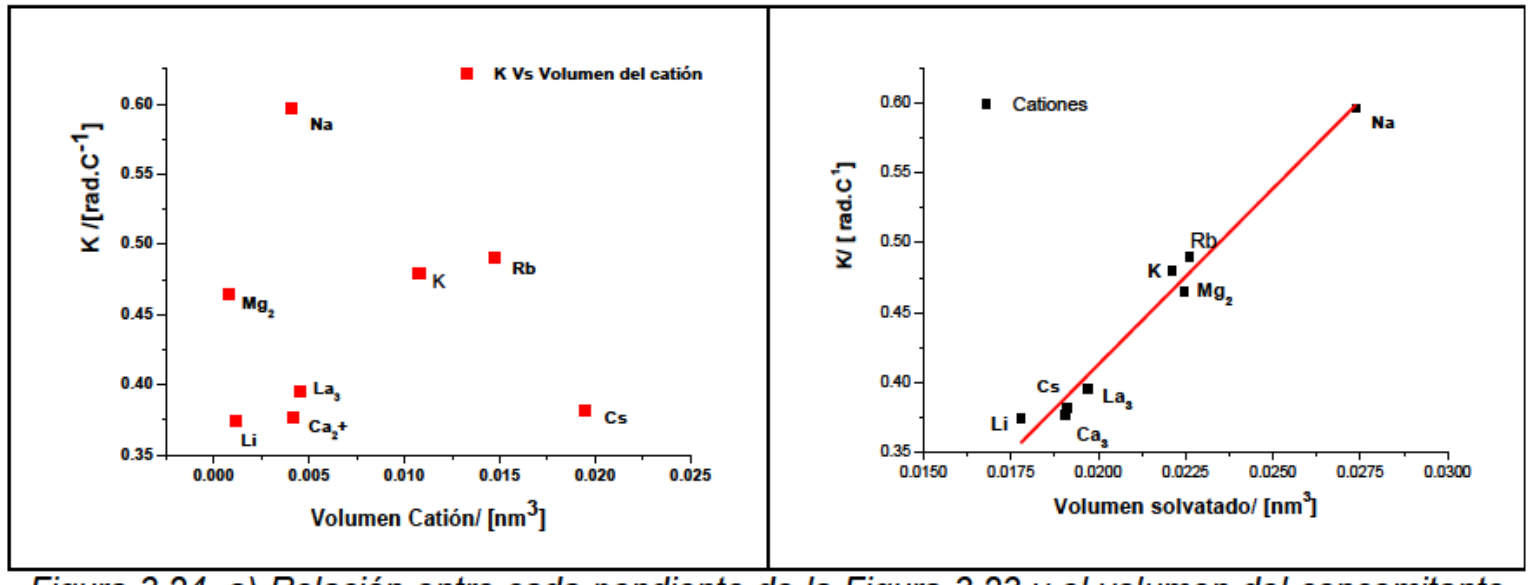

Figura 3.24. a) Relación entre cada pendiente de la Figura 3.23 y el volumen del concomitante catión intercambiado (b) Las relaciones entre cada pendiente de la Figura 3.23 y el volumen aparente solvatado (catión intercambiado más las moléculas de agua calculada).

El disolvente intercambiado también podría ser considerado como un número de hidratación aparente por unidad DBS $^{-}$dentro de la película o por electrón intercambiados durante la reacción. Los números de hidratación aparentes por unidad de carga en la película reducida resultantes son : 0.7 para $\left(\mathrm{pPy} / \mathrm{DBS}^{-}\right)^{\mathrm{n}}\left(\mathrm{Na}^{+}\right)_{n}, 0.5$ para $\left(\mathrm{pPy} / \mathrm{DBS}^{-}\right)^{\mathrm{n}}\left(\mathrm{Li}^{+}\right)_{\mathrm{n}}, 0.36$ para $\left(\mathrm{pPy}^{2} / \mathrm{DBS}^{2-}\right)^{\mathrm{n}}\left(\mathrm{Mg}^{2+}\right)_{\mathrm{n}}, 0.4$ para $\left.\quad(\mathrm{pPy} / \mathrm{DBS})^{-}\right)^{\mathrm{n}}\left(\mathrm{K}^{+}\right)_{n}, 0.25$ para $\left(\mathrm{pPy} / \mathrm{DBS}^{2-}\right)_{n}\left(\mathrm{Ca}^{2+}\right)_{\mathrm{n}}, 0.25$ para $\left(\mathrm{pPy} / \mathrm{DBS}^{-}\right)^{\mathrm{n}}\left(\mathrm{Rb}^{+}\right)_{\mathrm{n}}$ y 0.2 para $\left(\mathrm{pPy} / \mathrm{DBS}^{3-}\right)^{\mathrm{n}}\left(\mathrm{La}^{3+}\right)_{\mathrm{n}}$ y 0.0 para $\left(\mathrm{pPy} / \mathrm{DBS}^{-}\right)_{\mathrm{n}}\left(\mathrm{Cs}^{+}\right)_{\mathrm{n}}$. 
Los Materiales reducidos pueden ser considerados como geles densos que tienen una composición (Tabla 3.5): (pPy/DBS $)^{n-}{ }_{s}\left(\mathrm{Li}^{+}\right)_{n} \mathrm{H}_{2} \mathrm{O}_{0.5 n} ; \quad(p P y / D B S)^{n-}{ }_{s} \quad\left(\mathrm{Na}^{+}\right)_{n} \quad \mathrm{H}_{2} \mathrm{O}_{0.7 n}$; $(\mathrm{pPy} / \mathrm{DBS})^{2 \mathrm{n}-}{ }_{\mathrm{s}}\left(\mathrm{Mg}^{2+}\right)_{\mathrm{n}} \mathrm{H}_{2} \mathrm{O}_{0.35 n} ;(\mathrm{pPy} / \mathrm{DBS})^{\mathrm{n}-}{ }_{\mathrm{s}}\left(\mathrm{K}^{+}\right)_{\mathrm{n}} \mathrm{H}_{2} \mathrm{O}_{0.4 n} ;(\mathrm{pPy} / \mathrm{DBS})^{2 \mathrm{n}-}{ }_{\mathrm{s}}\left(\mathrm{Ca}^{2+}\right)_{\mathrm{n}} \mathrm{H}_{2} \mathrm{O}_{0.25 n}$; $(p P y / D B S)^{n-}{ }_{s}\left(\mathrm{Rb}^{+}\right)_{n} \mathrm{H}_{2} \mathrm{O}_{0.25 n} ;(\mathrm{pPy} / \mathrm{DBS})^{3 \mathrm{n}-}{ }_{s}\left(\mathrm{Ca}^{3+}\right)_{\mathrm{n}} \mathrm{H}_{2} \mathrm{O}{ }_{02 n} ;(\mathrm{pPy} / \mathrm{DBS})^{\mathrm{n}-}{ }_{s}\left(\mathrm{Rb}^{+}\right)_{\mathrm{n}} \mathrm{H}_{2} \mathrm{O}_{0.25 n}$.

\section{III.11.5 Caracterización sensora de los actuadores}

En los apartados anteriores se presentó la caracterización electroquímica de las películas poliméricas pPy/DBS autosoportadas, así como propiedades básicas de los actuadores de bicapa. En esta sección se presentan los resultados de los actuadores electroquimiomecánicos de bicapa pPy/DBS//cinta (músculos) actuando en diferentes condiciones de corriente, temperatura y concentración, con el fin de intentar estudiar, si, al igual que el material (película autosoportada), son capaces de sentir las condiciones experimentales a la vez que dicha bicapa se mueve, obteniendo de esta forma dispositivos duales actuadores/sensores ${ }^{163}$.

Para ello se estudió la relación que existe entre la evolución del potencial del dispositivo o el consumo de energía eléctrica durante la reacción electroquímica asociada al movimiento del músculo polimérico y los cambios de cada una de las variables físicas o químicas que actúan sobre la reacción.

\section{III.11.5.1 Caracterización del actuador como sensor de la densidad de corriente aplicada}

Se caracterizó el actuador bicapa pPy/DBS//cinta mediante ondas cuadradas consecutivas de corriente constante $( \pm 1.5, \pm 3, \pm 4.5, \pm 6, \pm 9, \pm 12$ y $\pm 15 \mathrm{~mA})$ consumiendo cargas anódicas y catódicas constantes e iguales en todos los casos hasta obtener respuestas cronopotenciométricas estacionarias, en un medio electrolítico acuoso de $0.1 \mathrm{M}$ de $\mathrm{LiClO}_{4}$, a la temperatura ambiental de $25^{\circ} \mathrm{C}$. Cada corriente fue aplicada durante el tiempo necesario para alcanzar un desplazamiento de $\pm 30^{\circ}$. Se puede observar que, para cada experimento, se produce un salto inicial del potencial tanto mayor cuanto mayor es la corriente aplicada. Le sigue un aumento lineal del potencial, con una mayor pendiente cuanto mayor es la corriente aplicada, Figura 3.25. Estos resultados son similares a los obtenidos para la película autosoportada pPy/DBS en el apartado 3.8. 

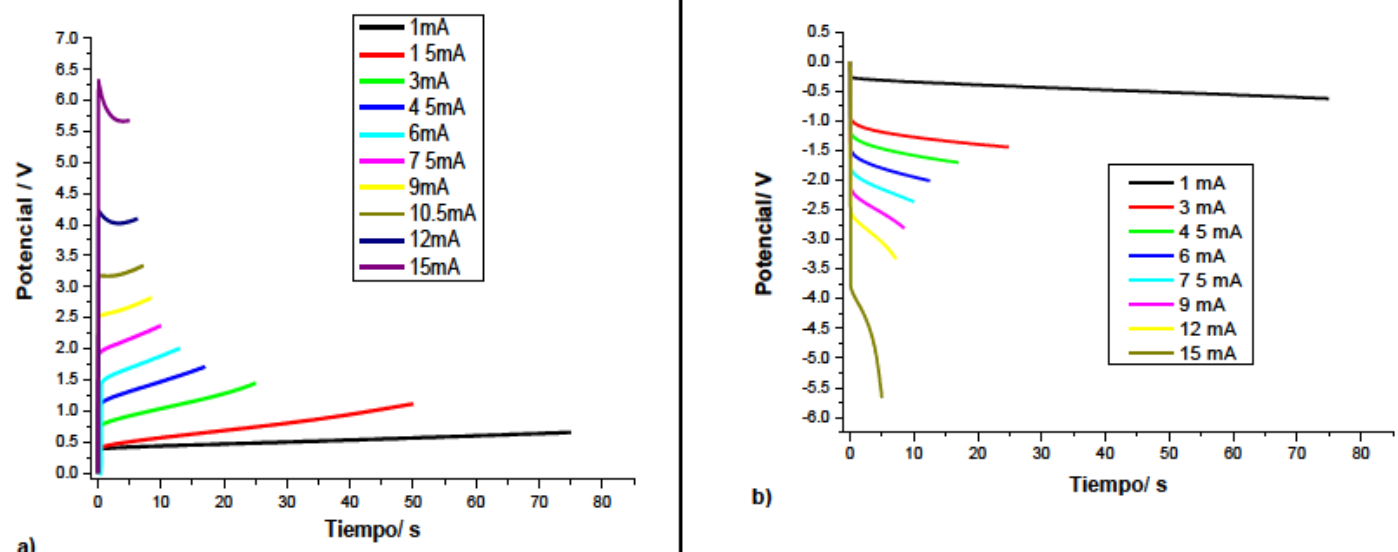

a)

Figura 3.25 Cronopontenciogramas obtenidos a un músculo bicapa pPy/DBS//cinta caracterizado: a) anódicas y b) catódicas aplicando diferentes corrientes durante el tiempo necesario para obtener desplazamientos de $30^{\circ}$ en una disolución acuosa $0.1 \mathrm{M} \mathrm{LiClO}_{4}$ a temperatura ambiente.

A medida que se incrementa la corriente, el tiempo necesario para realizar el recorrido de $30^{\circ}$ es menor: la velocidad angular del movimiento aumenta al aumentar la corriente eléctrica aplicada al actuador polimérico. La carga eléctrica consumida para efectuar un desplazamiento constante permanece prácticamente constante, tal y como se demostró en el apartado III.8 (Figuras 3.13), corroborando el comportamiento farádico de los actuadores.

\section{Consumo de energía eléctrica en función de la densidad de corriente}

De los cronopontenciogramas anódicos y catódicos obtenidos para el actuador bicapa pPy/DBS//cinta (Fig. 3.25) se calculó, por integración, el consumo de energía eléctrica en función de la corriente aplicada (ecuación 2.3). Como se puede observar en la Figura 3.26 la energía eléctrica consumida durante el movimiento sigue un incremento lineal con la corriente aplicada para un actuador pPy/DBS//cinta desplazándose un ángulo constante: la energía eléctrica consumida por el actuador es un sensor de la corriente. Dicha relación lineal se puede considerar una recta de calibración de un sensor de corriente, cuya pendiente (7025.44 J/(Ag) para corrientes anódicas), ($6149.06 \mathrm{~J} /(\mathrm{Ag})$ para corrientes catódicas) es la sensibilidad del sensor. 


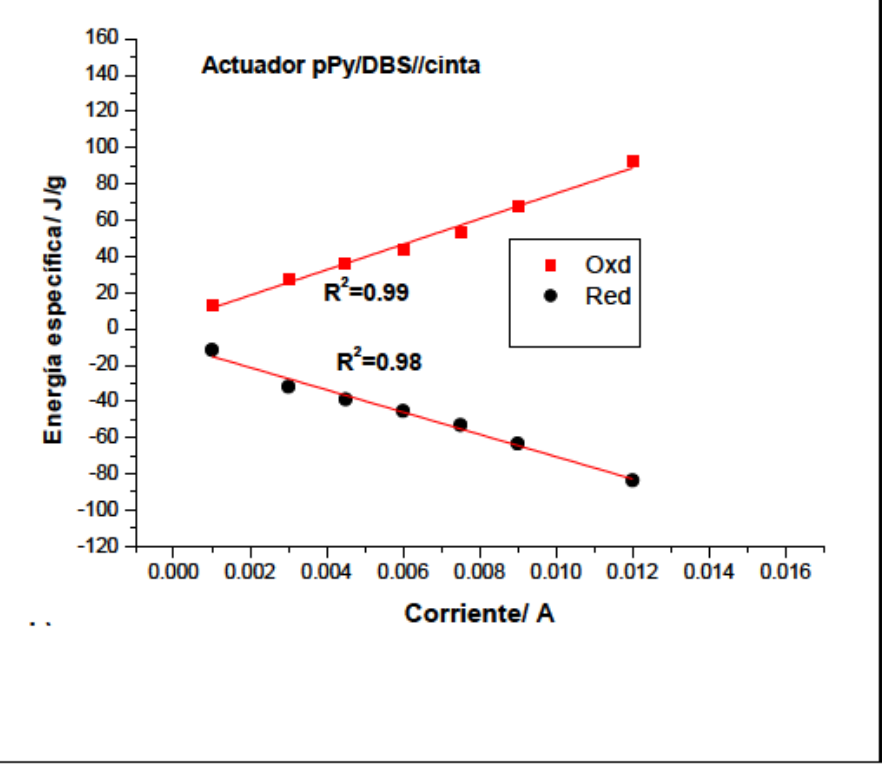

Figura 3.26 Evolución de la energía eléctrica consumida durante un desplazamiento de $30^{\circ}$ para diferentes densidades de corriente aplicadas, de un actuador pPy/DBS//cinta moviéndose en una disolución acuosa $0.1 \mathrm{M} \mathrm{LiClO}_{4}$ a temperatura ambiente.

\section{III.11.5.2 Caracterización del actuador como sensor de la temperatura}

Siguiendo la misma metodología del apartado anterior se caracterizaron los actuadores pPy/DBS//cinta a diferentes temperaturas de trabajo. Para ello se utilizó un criostato de circulación con un accesorio soporte para la celda electroquímica, tal y como se describió en el capítulo II, para estabilizar la temperatura del medio electrolítico a diferentes valores.

Continuando el mismo esquema experimental que en el caso anterior, se estudió el comportamiento del actuador pPy/DBS//cinta manteniendo constantes todas las variables experimentales, variando solamente la temperatura entre los diferentes experimentos. En la Figura 3.27 se muestra los cronopotenciogramas anódicos y catódicos estacionarios obtenidos por ondas cuadradas consecutivas de corriente de \pm $1.5 \mathrm{~mA}$ a las temperaturas de: $8,10,15,20,25,35,38^{\circ} \mathrm{C}$. 

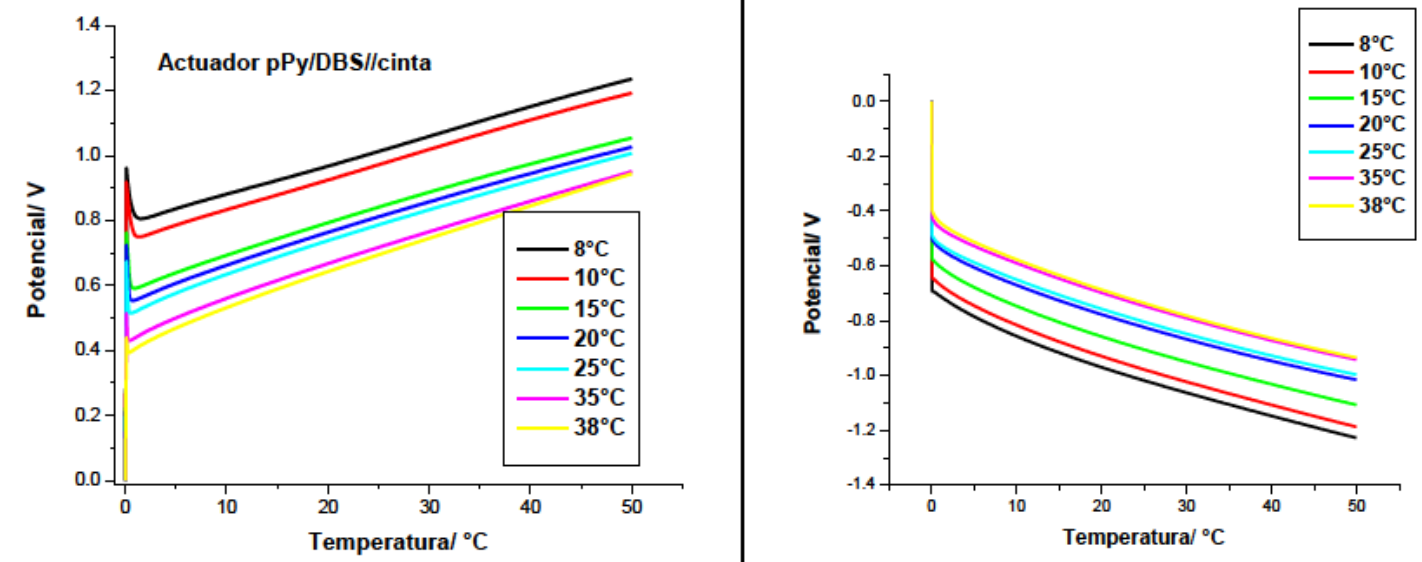

Figura 3.27 Cronopotenciogramas de evolución del potencial a diferentes temperaturas para un actuador pPy/DBS//cinta moviéndose durante $30^{\circ}$ en una disolución acuosa $0.1 \mathrm{M} \mathrm{LiClO}_{4}$ aplicando una corriente de $\pm 1.5 \mathrm{~mA}$.

Los resultados obtenidos son similares a los obtenidos para las películas autosoportadas pPy/DBS: el potencial muscular evoluciona durante la actuación a valores mayores al disminuir la temperatura (Figura 3.27). Ello se debe a que a medida que disminuye la temperatura, la energía térmica disponible para llevar a cabo la reacción electroquímica también disminuye. Por tanto, se requiere un aumento en el consumo de energía eléctrica para que se pueda realizar la reacción: siendo el flujo de corriente constante a cada tiempo de flujo una mayor energía requiere un mayor potencial muscular.

\section{Consumo de energía eléctrica en función de la temperatura}

Integrando los cronopotenciogramas estacionarios obtenidos a diferentes temperaturas se calcula la energía eléctrica consumida por los actuadores poliméricos. En la Figura 3.28 se muestra la gráfica correspondiente a la evolución de la energía eléctrica consumida para realizar un desplazamiento constante de $30^{\circ}$ a diferentes temperaturas.

La energía eléctrica consumida para el actuador disminuye linealmente con el incremento de la temperatura: la energía consumida por el actuador es $\mathrm{n}$ sensor de la temperatura. Este resultado ratifica la dualidad del actuador polimérico como un sensor de temperatura a la vez que el actuador se está moviendo (es a la vez actuador y sensor de la temperatura). 


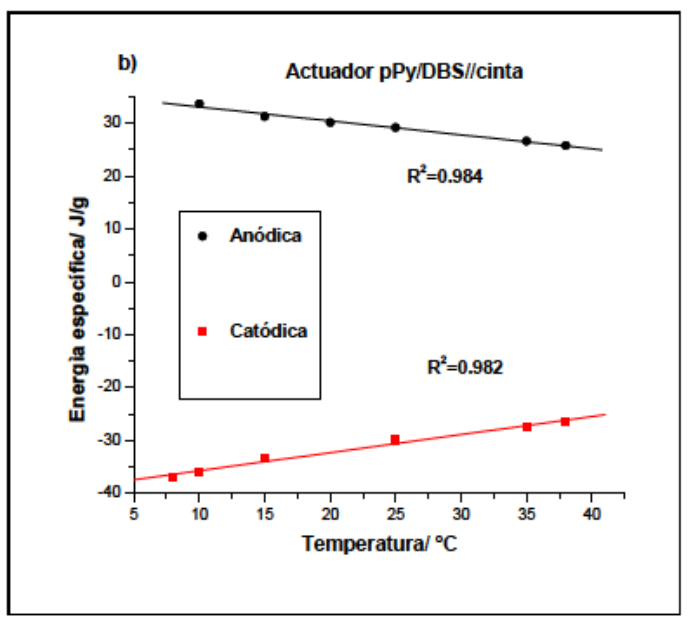

Figura 3.28 Evolución de la energía eléctrica consumida por un actuador pPy/DBS//cinta realizando en un movimiento de $30^{\circ}$ obtenida por integración de los cronopotenciogramas mostrados en la figura 3.27 en función de la temperatura a la que realizó el movimiento.

\section{III.11.5.3 Caracterización del actuador como sensor de la concentración del} electrolito

Se llevó a cabo un procedimiento experimental análogo a los realizados para diferentes corrientes aplicadas o a diferentes temperaturas, manteniendo constantes dichas variables experimentales y variando para cada uno de los experimentos la concentración del electrolito en el que están inmersos los actuadores poliméricos pPy/DBS//cinta. Para este fin, se trabajó en condiciones galvanostáticas aplicando ondas cuadradas consecutivas de $\pm 1 \mathrm{~mA}$ durante un tiempo de 50 s cada una $( \pm 50$ $\mathrm{mC}$ ) para obtener un desplazamiento de $\pm 30^{\circ}$, hasta conseguir respuestas cronopotenciométricas estacionarias, a $25^{\circ} \mathrm{C}$ en disoluciones acuosas con diferentes concentraciones $(0.04,0.06,0.08,0.1,0.15,0.3,0.6$, y $1.0 \mathrm{M})$ de $\mathrm{LiClO}_{4}$.

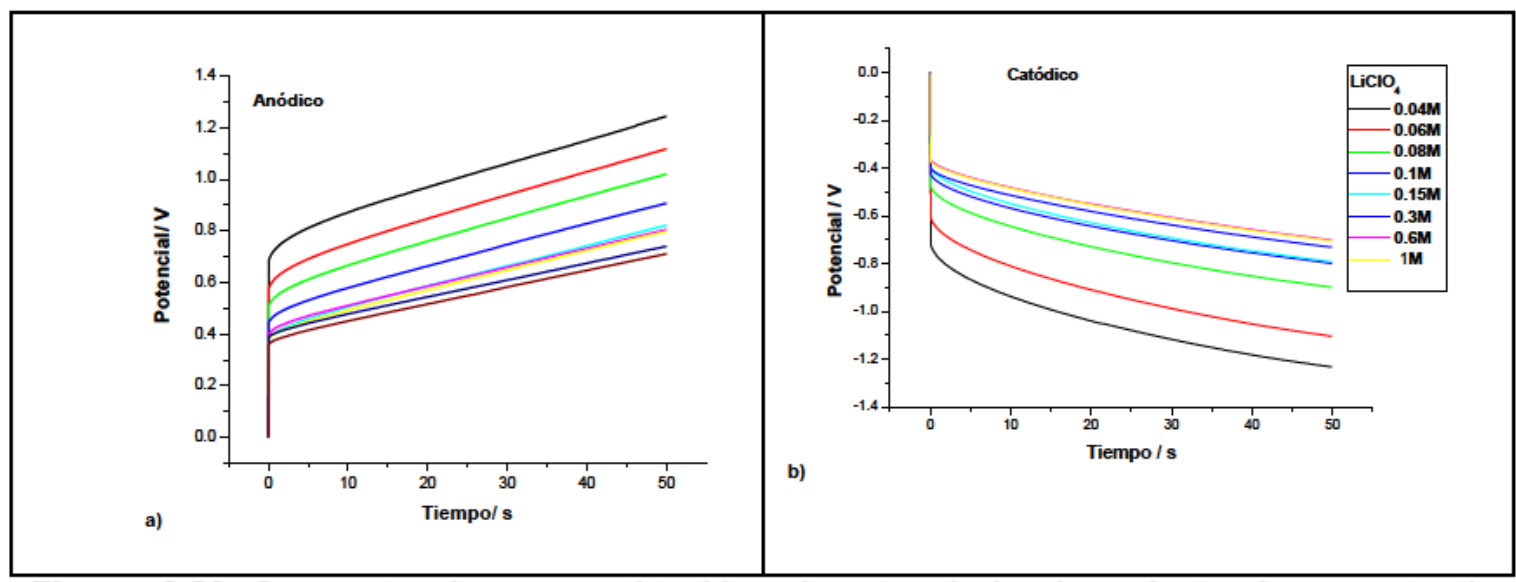

Figura 3.29 Cronopotenciogramas obtenidos durante el desplazamiento de un actuador $\mathrm{pPy} / \mathrm{DBS} / /$ cinta aplicando una corriente de $\pm 1 \mathrm{~mA}$ en una disolución acuosa con diferentes concentraciones de $\mathrm{LiClO}_{4}$ :a temperatura ambiente a) anódicos y b) catódicos. 
Las Figuras 3.29 a y b muestran las respuestas cronopotenciométricas estacionarias, anódicas y catódicas, respectivamente, obtenidas. Son análogas a las obtenidos con la película autosoportada (Figuras 3.17): la evolución del potencial muscular se desplaza a valores mayores a medida de que se va disminuyendo la concentración del electrolito. Estos resultados corroboran que la reacción 1.2 es la reacción que controla el movimiento: cuando hay menor concentración de uno de los reactivos (menor energía química), es necesario consumir una mayor energía eléctrica para llevar a cabo la reacción ${ }^{163,214}$

\section{Consumo de energía eléctrica en función de la concentración del electrolito}

En la Figura 3.30a se presenta la respuesta del consumo de energía específica de un actuador pPy/DBS//cinta movido por la corriente anódica y por la corriente catódica Fig 3.30b. Al aumentar las concentraciones de la disolución, se observa la disminución de energía específica consumida por el actuador para llevar a cabo el movimiento.

a)

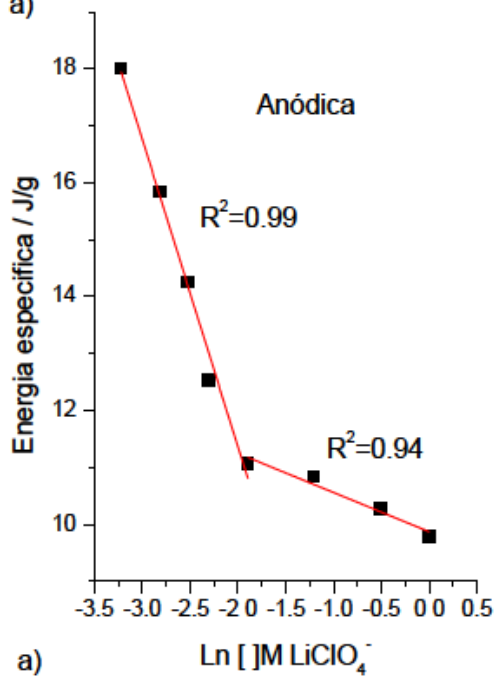

b)

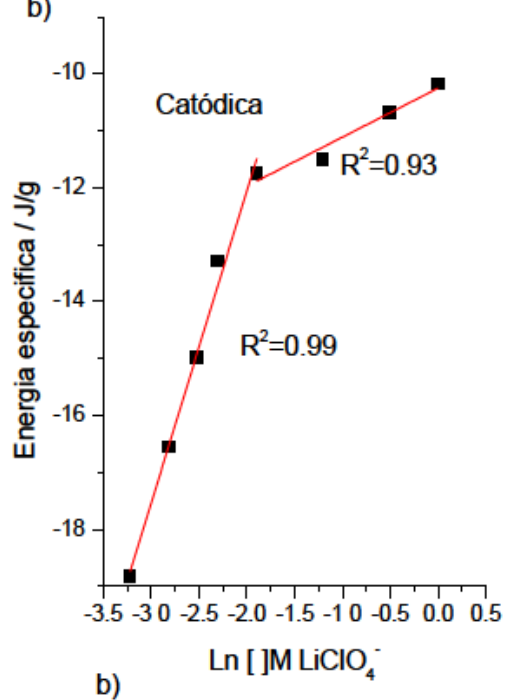

Figura 3.30 Variación del consumo de energía específica de un actuador pPy/DBS//cinta en respuesta al desplazamiento de $30^{\circ}$ en soluciones acuosas de $\mathrm{LiClO}_{4}$ obtenidos de la figura 3.29.

La variación de la energía específica en respuesta del trabajo realizado para desplazarse $30^{\circ}$ a diferentes concentraciones da lugar a dos regiones lineales mostrando que la evolución del gasto de energía específica está relacionado con el logaritmo de la concentración; respuestas similares se han reportado para otros polímeros conductores ${ }^{221}$. 
En las regiones lineales, tanto en la parte anódica como en la catódica, se ve que la energía específica se incrementa a medida de que se va disminuyendo la concentración del electrolito: la energía eléctrica consumida es un sensor de las condiciones químicas de actuación. El actuador es un sensor semilogarítmico de concentración para esas dos regiones diferenciadas por una sensibilidad diferente: diferentes pendientes. El actuador es a la vez actuador y sensor de la concentración. 


\section{III.11.6 Deslizamiento}

Los Músculos artificiales basados en polímeros conductores cumplen la mayoría de las características requeridas para su utilización en el desarrollo de nuevos robots, ya sea como elementos sensores, como motores o como motores/sensores 0 actuadores/sensores, tal y como se ha visto en los apartados anteriores. Estos motores todavía presentan ciertos problemas que necesitan ser estudiados, comprendidos y resueltos como, por ejemplo, la presencia de efectos de deslizamiento (creeping) de las posiciones inicial y final al someter el motor a ciclos consecutivos: el ángulo descrito es constante pero se va desplazando con cada ciclo un pequeño ángulo, generalmente en sentido de las agujas del reloj. Estos efectos de deslizamiento requieren procesos de control de auto-compensación complejos para obtener motores seguros, fiables, reproducibles y fáciles de controlar. Un conocimiento más preciso del origen de estos efectos podría en un futuro permitir su eliminación o, al menos, su compensación teórica más fácil con un microprocesador sencillo. A día de hoy no son muchos los esfuerzos que se han hecho en esta dirección.

Por ello, en este apartado, se pretende estudiar el origen físico-químico de ese deslizamiento en los actuadores de pPy/DBS. Se procedió a estudiar el comportamiento de una bicapa pPy/DBS//cinta mediante voltamperometría cíclica entre los potenciales:-2,5 y $1 \mathrm{~V}$ a $6 \mathrm{mV} / \mathrm{s}$ en disoluciones acuosas $0.1 \mathrm{M} \mathrm{LiClO}_{4}$ a temperatura ambiente, grabando en vídeo, simultáneamente, el movimiento angular descrito por el actuador durante varios ciclos consecutivos de potencial. La respuesta voltamperométrica estacionaria del actuador después de ser sometido a varios ciclos de potencial (Figura 3.31 a) es similar a las que ya se han mostrado durante el desarrollo de esta tesis, ya sea para las películas autosoportadas como para las bicapas.

Por integración de la respuesta voltamperométrica se obtuvo la evolución de la carga consumida durante el barrido de potencial (respuesta culovoltamétrica, carga/potencial o Q/V) por las reacciones pPy/DBS (Figura. 3.31b). Los puntos 1 a 10 se correlacionan con los del voltamperograma (Figura. $3.31 \mathrm{a}$ ).

Los fotogramas de vídeo muestran en las imágenes C1 a C10 de la Figura 3.31c la posición angular del músculo en los puntos 1 a 10, respectivamente. El ángulo descrito por la parte inferior de la bicapa se midió desde el fotograma correspondiente a cada potencial muscular, siguiendo el procedimiento descrito en el apartado II.8

Tras un ciclo completo de potencial, el dispositivo no recupera su posición inicial, originándose un deslizamiento continuo de la posición inicial y final del movimiento en 


\section{Capítulo III}

los sucesivos ciclos de actuación, en el sentido de las agujas del reloj como se puede observar (con dificultad) de los fotogramas inicial y final (Figuras $3.31 \mathrm{c} 1$ y c10).

Combinando las respuestas culovoltamétricas y los ángulos medidos de los fotogramas grabados se obtienen las respuestas culodinámicas (carga/ángulo, $Q / \alpha$ ), figura 3.31d. En ella se observa más claramente el deslizamiento angular positivo sufrido durante el ciclo de potencial desde el punto inicial (1) al punto final (10), correspondientes al mismo potencial. También se puede ver que al inicio del barrido anódico hay un consumo de corriente catódica, del punto 1 al punto 2, pero sin deslizamiento angular: carga en una reacción irreversible que no produce deslizamiento. Una vez que continúa el barrido anódico, comienza la oxidación de la película de pPy/DBS, consumiendo una carga positiva y observando un movimiento en el sentido esperado para la reacción de oxidación: disminuye su volumen y tira de la bicapa hasta el final del barrido anódico (Figura 3.31 c2 a Figura 3.31 c5, puntos 2-5 de la Figura. 3.31d). Al inicio del barrido catódico, el material continúa oxidándose como se hizo evidente al analizar la carga consumida y el sentido del deslizamiento, obteniendo un deslizamiento angular máximo de 145 grados (Figura 3.31 c5 a f3.31. c6, puntos 5-6 de la Figura 3.31d). Siguiendo con el barrido catódico, comienza la reducción, y tanto la carga como el ángulo tienen la evolución contraria a la que tuvieron durante la oxidación (3.31 c6 a 3.391 c10, puntos 6-9 de la figura 3.31d). Sin embargo al final del barrido catódico ni la carga ni el ángulo vuelven al valor inicial: hay un consumo irreversible total de carga (puntos 1-10 de la figura 3.31d) y el músculo no recupera la posición inicial (punto 1).

A pesar de la carga consumida en procesos irreversibles, el motor muscular mantiene su naturaleza farádica: los ángulos descritos son funciones lineales de la carga consumida, como se observa en la respuesta culodinámica (Figura 3.31.d).

Desde un punto de vista de la ingeniería son motores muy robustos debido a su naturaleza farádica, corroborando los resultados obtenidos en los apartados anteriores utilizando músculos de diferentes geometrías: la posición del motor está bajo el control lineal de la carga consumida y la velocidad de movimiento está bajo el control lineal de la corriente aplicada al actuador. Tanto la carga como el ángulo siguen una evolución muy similar describiendo un movimiento angular de flexión de 145 grados por ciclo, sin embargo este se desliza 15 grados por ciclo en sentido positivo, ángulo del punto 1 hasta ángulo del punto 10 (efecto deslizamiento). Este Deslizamiento positivo es, aproximadamente un $10 \%$ del deslizamiento total y corresponde con un $10 \%$ de carga irreversible respecto a la carga total del ciclo, apuntando a la carga irreversible como posible origen de la actuación irreversible de la película pPy/DBS. Esta carga irreversible fue atribuida ${ }^{193-196}$ a la formación del ácido (HDBS) durante el barrido 


\section{Capítulo III}

anódico y la subsiguiente descarga de los protones para formar hidrógeno al llegar el barrido a los potenciales más catódicos.

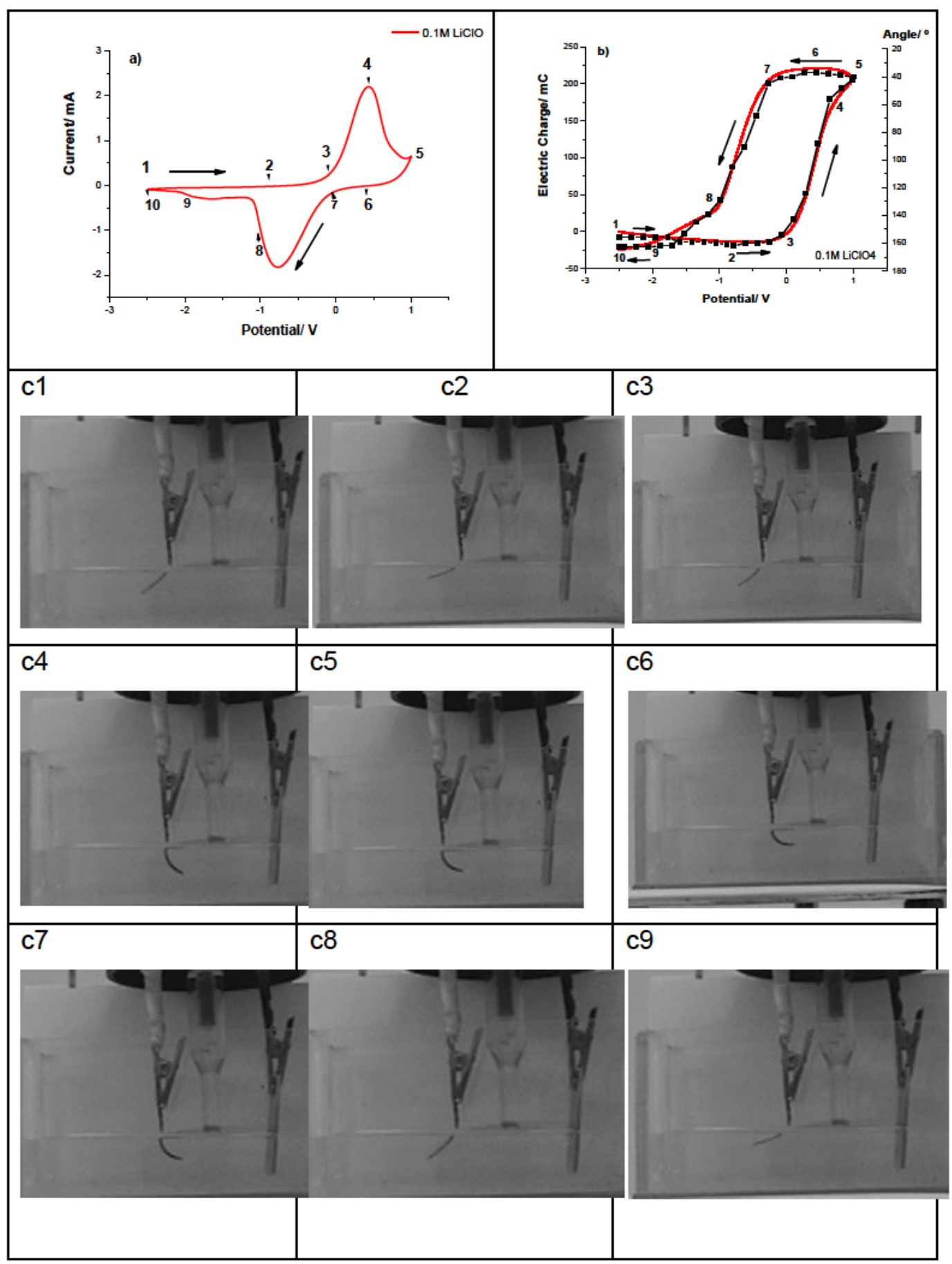




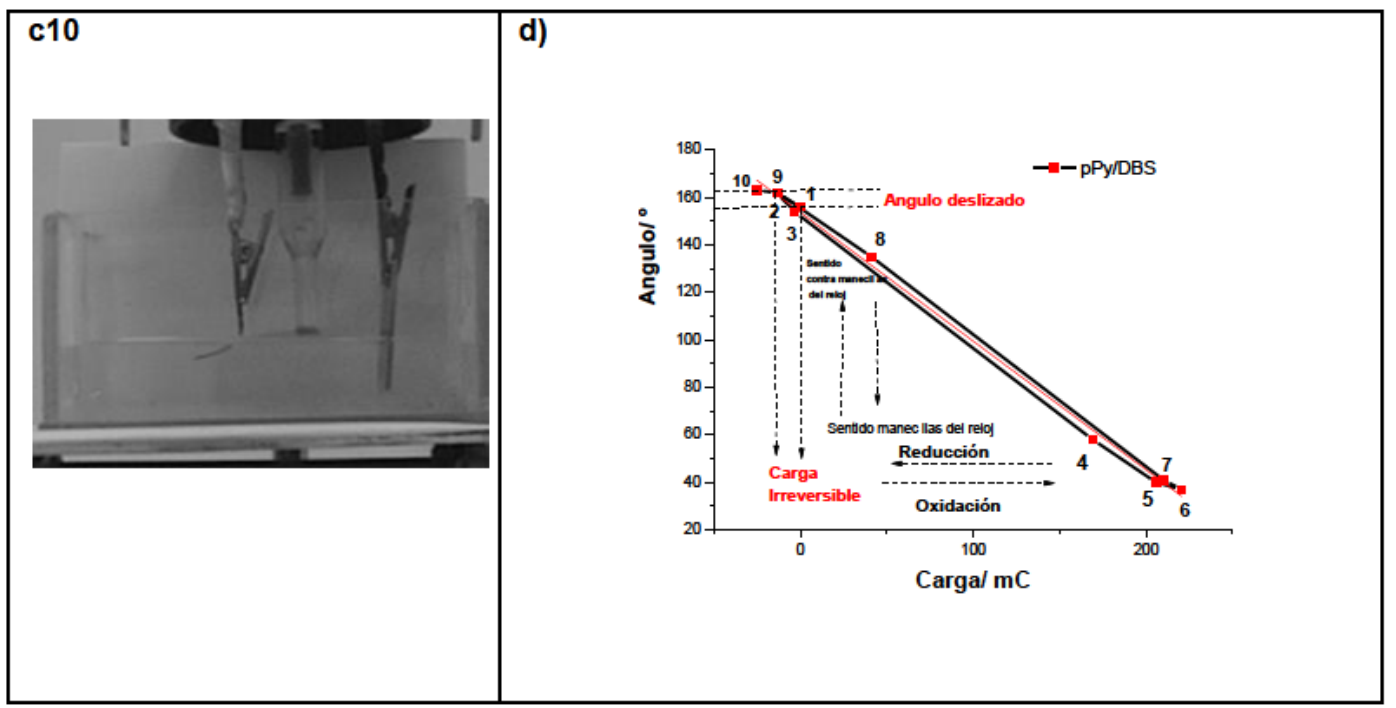

Figura 3.31. (a) Respuesta voltamperométrica (V/i) de un actuador pPy/DBS como electrodo de trabajo en una solución acuosas $0.1 \mathrm{M} \mathrm{LiClO}_{4}$, después de 3 ciclos de estabilización consecutivos, cuando se somete a ciclos potenciales de $-2,5 \mathrm{~V}$ a $1 \mathrm{~V}$, de $-1.2 \mathrm{~V}$ a $0.6 \mathrm{~V}$ o de $-1 \mathrm{~V}$ a $0.4 \mathrm{~V}$, a una velocidad de barrido de (6 mV/s). (b) respuestas culovoltamétricas (QN) obtenida por integración de los voltamperogramas de (a), (c). Las fotos de c1 a c10 muestran la posición de flexión del músculo correspondiente a los puntos del 1 al 10. (d) Evolución de la carga consumida, de la respuesta culovoltamétrica y evolución paralela del ángulo descrito por el músculo.

Las respuestas culovoltamétricas (Figura 3.31b) también permiten obtener información acerca de los cambios estructurales que ocurren en el polímero conductor durante las reacciones de oxidación y reducción. Cualquier variación de pendiente $Q / E$ significa un cambio en la velocidad de la reacción. Los distintos cambios de pendiente en las ramas anódica y catódica estarán relacionados con cambios estructurales en la película pPy/DBS, De las intersecciones de las pendientes se obtendrán sus posibles rangos de potencial y las cargas involucradas en cada uno de los cambios estructurales ${ }^{199}$. La Figura $3.31 \mathrm{~b}$ corrobora los procesos de la película pPy/DBS: reducción-expansión, reacción (1.2) hacia atrás (Apartado III.5) que pasa entre el potencial inicial (punto 1) y el mínimo QV, punto 2: la evolución negativa de la caga entre ambos puntos indica la reducción y el incremento paralelo positivo del ángulo descrito indica expansión de la película de pPy/DBS. Desde el punto 2 al 4 hay una rápida (pendiente alta) oxidación (deslizamiento positivo de la carga) siguiendo la reacción 1 con expulsión de cationes y agua, que produce la contracción del polímero conductor (incremento negativo del ángulo): oxidación-contracción. En el punto 4 se inicia otro cambio de pendiente, disminuyendo bruscamente la velocidad de oxidación del polímero debido a la oxidación-compactación. El punto de separación entre la oxidación-reducción y oxidación-compactación es el potencial de cierre: la distancia media entre las cadenas iguala el diámetro de los cationes que salen y la estructura se cierra atrapando un gran porcentaje de los contra-iones (cationes). Éste es un material 
blando y húmedo y la oxidación con sigue a sobretensiones crecientes con velocidades mucho más bajas: los iones tienen que apartar las cadenas poliméricas par abrir su camino hacia la disolución. En su camino dragan las moléculas de agua que se encuentran (electroósmosis) y el espacio se cierra detrás de ellos formando una estructura conformacional cada vez más compactada. El proceso de oxidacióncompactación sigue durante el inicio del barrido catódico de potencial hasta el máximo, pinto 6 de la figura 3.31b: la sobretensión de oxidación es todavía alta y el material no ha alcanzado su equilibrio de oxidación.

Después del punto máximo QV la reducción de la película comienza con un proceso de reducción-relajación muy lento (puntos 6-7), seguido por el rápido proceso de reducción-expansión (punto 7 a 8 ). En el punto 8 cae de nuevo, bruscamente la velocidad de reducción de la película de polímero conductor (puntos 8 a 10). Esta brusca disminución de la velocidad es atribuida a la formación de nano-vesículas (agua e iones rodeados por una capa DBS) ${ }^{192,193,196}$ en la película, similar a los observados en dispersiones coloidales del tensoactivo ${ }^{222 . ~ L a ~ c a r g a ~ d e ~ l a ~ r e d u c c i o ́ n ~}$ (Figura 3.31b línea completa) siempre impulsa el incremento de volumen de la película pPy/DBS, debido a la entrada de cationes y agua dando un deslizamiento angular en paralelo (Figura 3.31b línea punteada).

El proceso de reducción lento a potencial catódico (de 9 a 10 y de 1 a 2) se solapa con la presencia paralela de reacciones electroquímicas irreversibles, evidenciada por la parte abierta de la respuesta $\mathrm{Q} / \mathrm{V}$ (Figura 3.31b) y atribuido a la reducción DBSH con lento desprendimiento de hidrógeno ${ }^{200,201}$. La diferencia de carga entre los puntos inicial y final de la respuesta QE es la carga irreversible (consumida por la reacción irreversible). Cabe notar que la presencia de la liberación de hidrógeno no produce un aumento notable de las corrientes catódicas para sobrepotenciales catódicos elevados en la respuesta voltamperométrica ${ }^{200,201}$ Tampoco se observa a simple vista el desprendimiento de burbujas desde el electrodo polimérico.

\section{III.12 Actuador tricapa pPy/DBS//cinta//pPy/DBS}

Pare el estudio y caracterización de los músculos artificiales de bicapa pPy/DBS//cinta abordados en el apartado anterior es necesaria la utilización de un contraelectrodo metálico para cerrar el circuito y permitir el flujo de corriente eléctrica a través del músculo. En dicho contraelectrodo se producen reacciones que generan productos que pueden degradar el funcionamiento del polímero conductor y, sobre todo, consumen mucha energía en procesos electroquímicos, como por ejemplo la electrolisis del agua. El objetivo de los músculos artificiales 
es transformar energía eléctrica en energía mecánica: si gran parte de la energía se consume en el contraelectrodo la eficiencia de la transformación siempre será muy baja. Con el fin de evitar dichas reacciones, obteniendo así un dispositivo mucho más eficiente desde el punto de vista energético, se construyeron tricapas. 223-225. En esta tesis se estudió el comportamiento de un actuador tricapa basado en el material estudiado, el pPy/DBS. De esta forma, se han construido actuadores tricapa $\mathrm{pPy} / \mathrm{DBS} / / \mathrm{cinta} / / \mathrm{pPy} / \mathrm{DBS}$ tal y cómo se ha especificado en la parte experimental. Se abordará aquí su caracterización electro-quimio-dinámica, así como el estudio y caracterización de su capacidad actuadora-sensora, de forma similar a cómo se estudiaron y caracterizaron las bicapas en los apartados anteriores.

\section{III.12.1. Respuesta voltamperométrica}

El actuador de tricapa se sometió a barridos consecutivos de potencial en la ventana de $-0.8 \mathrm{~V}$ a $0.3 \mathrm{~V}$ a una velocidad de barrido de $5 \mathrm{mV} / \mathrm{s}$ en disolución acuosa $0.1 \mathrm{M}$ de $\mathrm{LiClO}_{4}$. Para ello la salida del electrodo de trabajo del potenciostato estaba conectada a una de las pPy/DBS y la salida del contraelectrodo y electrodo de referencia estaban conectados (cortocircuitados) a la otra película de pPy/DBS que conforman la tricapa. La película de dos caras adhesivas intermedia garantiza el aislamiento eléctrico entre las dos películas de pPy-DBS evitando el cortocircuito entre el electrodo de trabajo y el contraelectrodo. El circuito se cierra a través de la disolución electrolítica.

La respuesta voltamperométrica del músculo se muestra en la Figura 3.32, se observa el máximo de oxidación a $0.047 \mathrm{~V}$ y el pico de reducción a $-0.547 \mathrm{~V}$, picos redox ubicados a valores de potencial similares a los obtenidos tanto para el material como para la bicapa pPy/DBS//cinta (Figuras 3.6 y 3.21 )

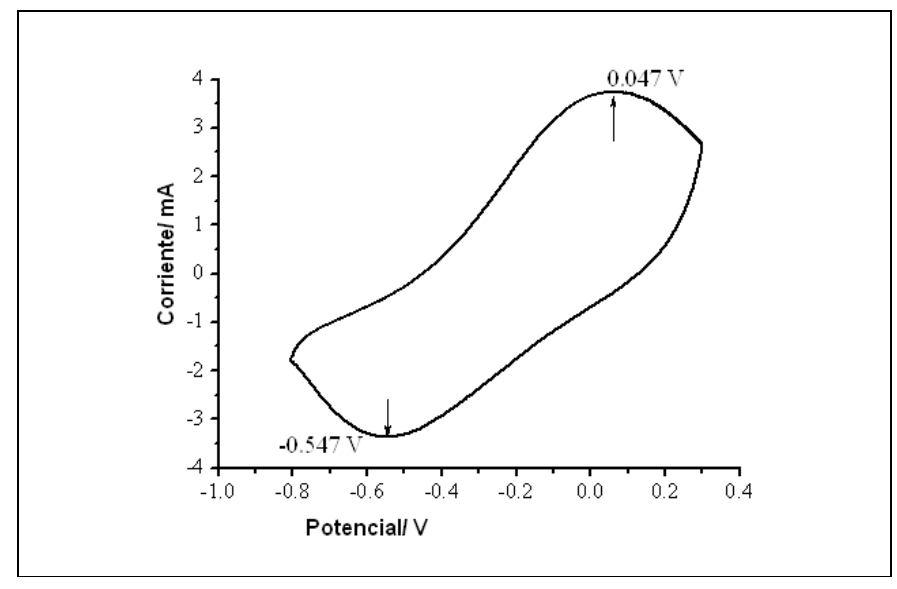

Figura.3.32. Voltamperograma registrado de un actuador tricapa $p P y / D B S / /$ cinta//pPy/DBS a una velocidad de barrido de $5 \mathrm{mV} / \mathrm{s}$ entre los límites potenciales de $-0.8 \mathrm{~V}$ y $0.3 \mathrm{~V}$ utilizando disolución acuosa $0.1 \mathrm{M} \mathrm{LiClO}_{4}$ como electrolito a temperatura ambiente. 


\section{Capítulo III}

\section{III.12.2 Respuesta dual actuadora/sensora tricapa pPy/DBS//cinta//pPy/DBS}

Se ha estudiado también, si, al igual que con el material pPy/DBS y el actuador bicapa pPy/DBS//cinta, el actuador tricapa pPy/DBS//cinta//pPy/DBS es capaz de sentir las variables de su entorno de trabajo mientras se mueve (sistema dual actuador-sensor). Para ello se utilizó el mismo procedimiento experimental utilizado en el estudio de las películas autosoportadas pPy/DBS y el de bicapas pPy/DBS//cinta.

Antes de someter a la tricapa a ondas cuadradas consecutivas de corriente, se aplicó una corriente eléctrica constante de $-0.01 \mathrm{~mA}$ durante 250 segundos. A continuación se sometió la tricapa a pulsos cuadrados consecutivos de corriente eléctrica. En distintos experimentos se aplicaron diferentes corrientes, siempre durante el tiempo necesario para describir un movimiento angular constante de $\pm 45^{\circ}$ por consumo de una carga constante. Se registraron las respuestas cronopotenciométricas durante los ciclos de oxidación/reducción consecutivos siguiendo el procedimiento experimental descrito en el apartado II.6.2 hasta conseguir respuestas estacionarias.

La dirección del movimiento angular del actuador tricapa pPy/DBS//cinta//pPy/DBS indica que la actuación de la película de polipirrol conectada como cátodo empuja el dispositivo (se expande por entrada de cationes al reducirse) y la que actúa como el ánodo tira del dispositivo (se contrae por expulsión de cationes al oxidarse) como se indica en la Figura 3.33. Esto significa que la actuación del músculo de tricapa está controlada por la reacción (1.2) con los cationes como el intercambio iónico prevalente entre las películas y la solución. ${ }^{225,163}$

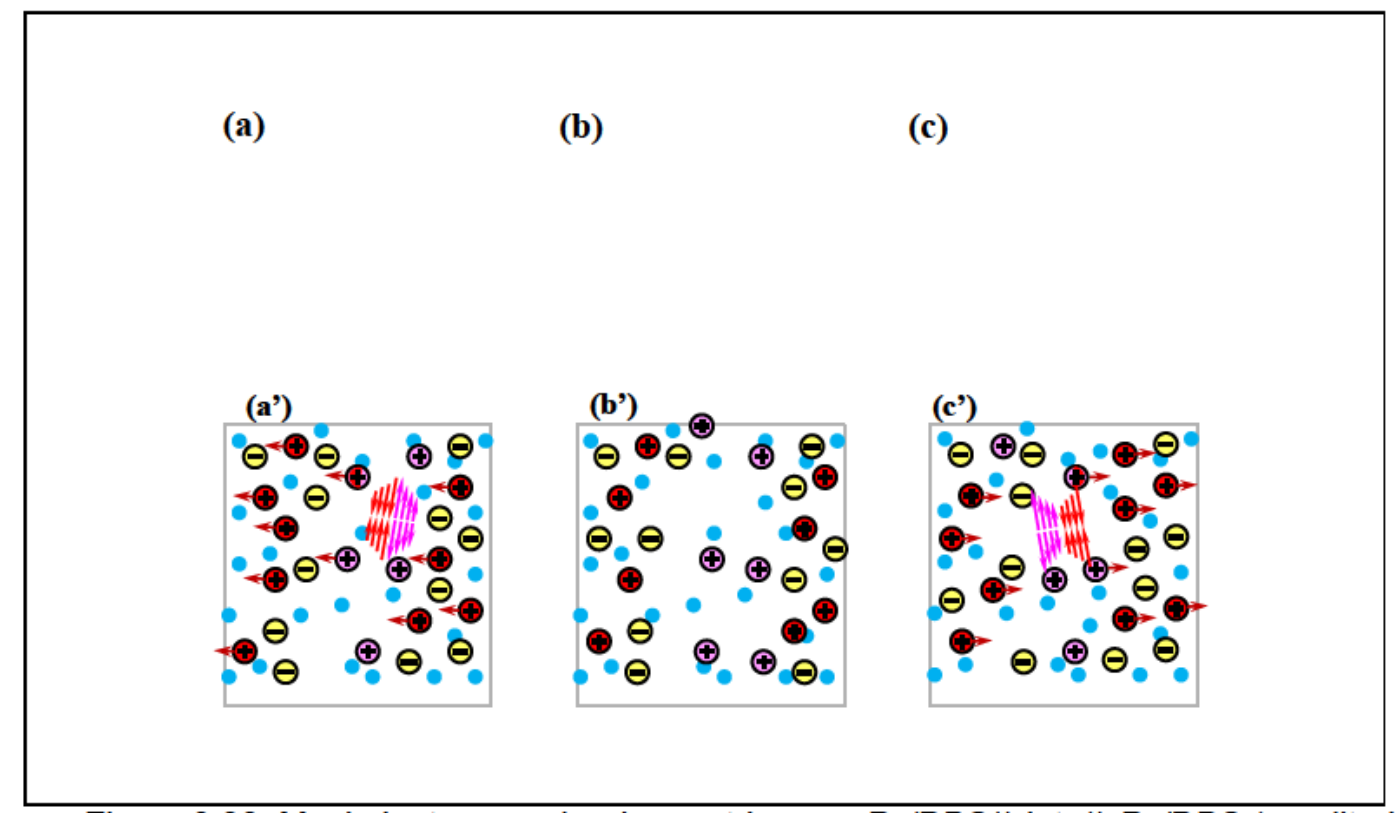

Figura 3.33. Movimientos angular de una tricapa $\mathrm{pPy} / \mathrm{DBS} / / \mathrm{cinta} / / \mathrm{pPy} / \mathrm{DBS}$ (amplitud, \pm 60 grados) en disolución. 
III.12.2.1 Influencia de la corriente eléctrica en el movimiento del Actuador tricapa pPy/DBS//cinta//pPy/DBS

Se estudió la capacidad de la tricapa pPy/DBS//cinta//pPy/DBS de sentir las condiciones eléctricas (corriente aplicada) mientras se mueve, de forma similar a como ocurría con las bicapas pPy/DBS//cinta. Para ellos se aplicaron diferentes ondas cuadradas de corrientes anódica y catódica constantes, desde $\pm 0.75 \mathrm{~mA}$ hasta $\pm 15 \mathrm{~mA}$. Se variaron los tiempos de aplicación de cada corriente, a fin de aplicar una carga constante y conseguir un deslizamiento angular constante de $\pm 45^{\circ}$ (para lo que se requirió un consumo de carga eléctrica constante de $60 \mathrm{mC}$ durante la reacción electroquímica (1.2)). Se mantuvieron constantes el resto de variables experimentales (temperatura ambiente y la concentración de la solución acuosa a $0.1 \mathrm{M}$ de $\mathrm{LiClO}_{4}$ ), obteniéndose la respuesta cronopotenciométrica estacionaria para cada una de las corrientes estudiadas.

La Figura 3.34 muestra las respuestas cronopotenciométricas normalizadas, anódicas y catódicas, de la tercera onda cuadrada consecutiva (estacionaria) tras la estabilización inicial para cada corriente aplicada. Al igual que con el material y la bicapa, tanto para los procesos de oxidación como de reducción, se obtiene un salto inicial de potencial debido a las diferentes resistencias presentes en la celda electroquímica, tras lo cual se observa un aumento del potencial tanto más rápido cuanto más elevada es la corriente aplicada.

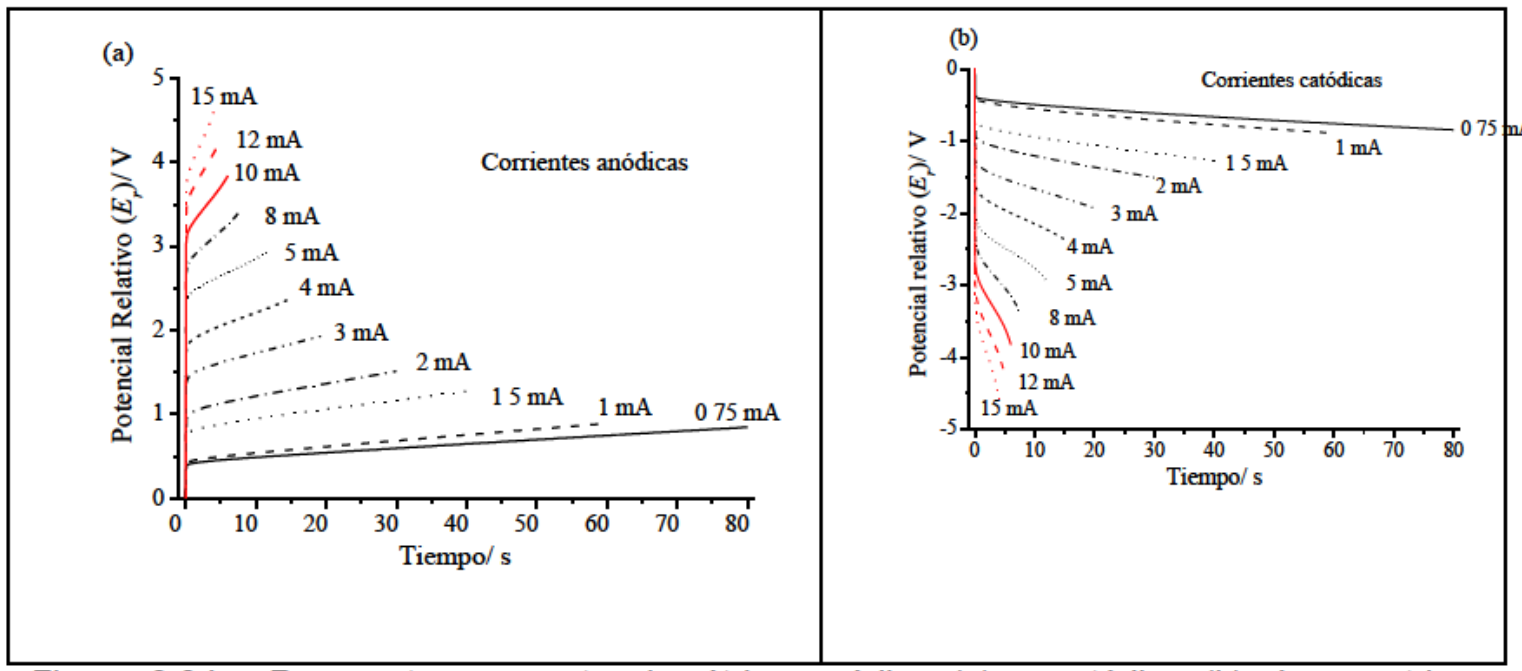

Figura 3.34. Respuesta cronopotenciométrica anódica (a) y catódica (b) de una tricapa pPy/DBS//cinta//pPy/DBS aplicando diferentes corrientes en una disolución acuosa $0.1 \mathrm{M}$ $\mathrm{LiClO}_{4}$ a temperatura ambiente durante el tiempo necesario para obtener movimientos angulares de $\pm 45^{\circ}$.

Se calculó la energía eléctrica consumida para describir los movimientos angulares de $\pm 45^{\circ}$ durante el flujo de las corrientes anódicas o catódicas aplicadas. La Figura 3.35 


\section{Capítulo III}

muestra que la energía eléctrica consumida para describir un movimiento angular constante $\left( \pm 45^{\circ}\right)$ es una función lineal de la corriente aplicada. De esta forma el actuador de triple capa puede percibir, durante el trabajo, cualquier cambio en la corriente eléctrica aplicada. Las pendientes obtenidas: $3.5116 \mathrm{~J} /(\mathrm{g} \mathrm{mA})$ y $-3.3873 \mathrm{~J} /(\mathrm{g}$ $\mathrm{mA}$ ) para corrientes anódicas y catódicas respectivamente indican la sensibilidad del actuador. Por lo tanto, el actuador tricapa es un sensor lineal de la corriente que fluye por él y las pendientes obtenidas son las sensibilidades de dicho sensor.

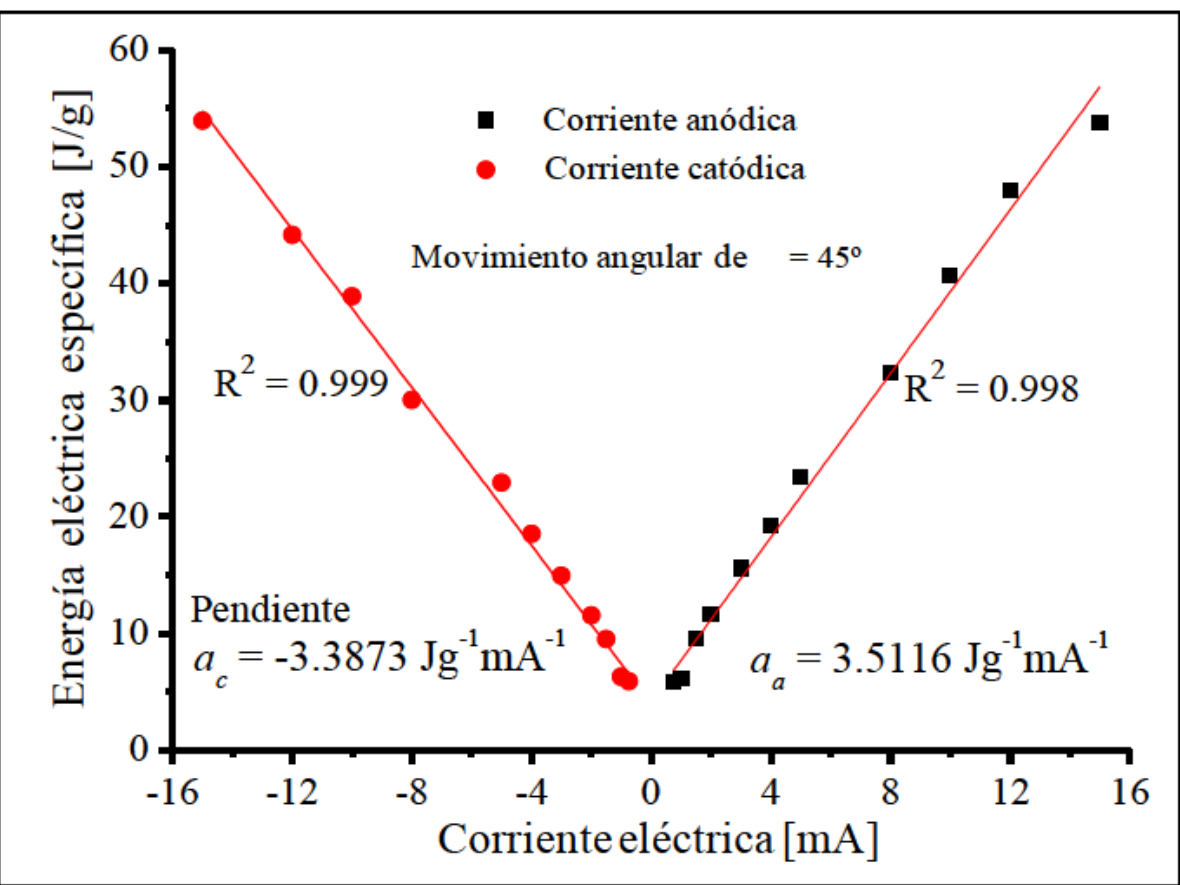

Figura 3.35.Energía eléctrica consumida por gramo de pPy/DBS en función de la corriente eléctrica aplicada obtenida por integración de los cronopotenciogramas de la Figura 3.34. $R^{2}$ es el coeficiente de correlación del ajuste lineal. a es la sensibilidad de los sensores anódicos (subíndice a) y catódicos (subíndice c); $\theta$ es el ángulo desplazado.

\section{III.12.2.2 Influencia de la temperatura en el movimiento del Actuador tricapa pPy/DBS//cinta//pPy/DBS}

Mediante el procedimiento experimental descrito en la Figura 2.5 se estudió la influencia de la temperatura, de $5^{\circ} \mathrm{C}$ a $45^{\circ} \mathrm{C}$, en las respuestas cronopotentiométricas bajo un flujo de las ondas cuadradas de corriente de $\pm 3 \mathrm{~mA}$ durante $20 \mathrm{~s}$ cada una (carga consumida $\pm 60 \mathrm{mC}$ ) en disolución acuosa $0.1 \mathrm{M} \mathrm{LiClO}_{4}$ que describe un movimiento angular reversible de $\pm 45^{\circ}$, como es de esperar para un motor farádico.

La Figura 3.35 muestra las respuestas cronopotenciométricas anódica (a) y catódica (b), respectivamente. Al igual que en los casos de la película autosoportada y la bicapa, el potencial muscular evoluciona a valores menores (menor resistencia) a medida que aumenta la temperatura de trabajo. 


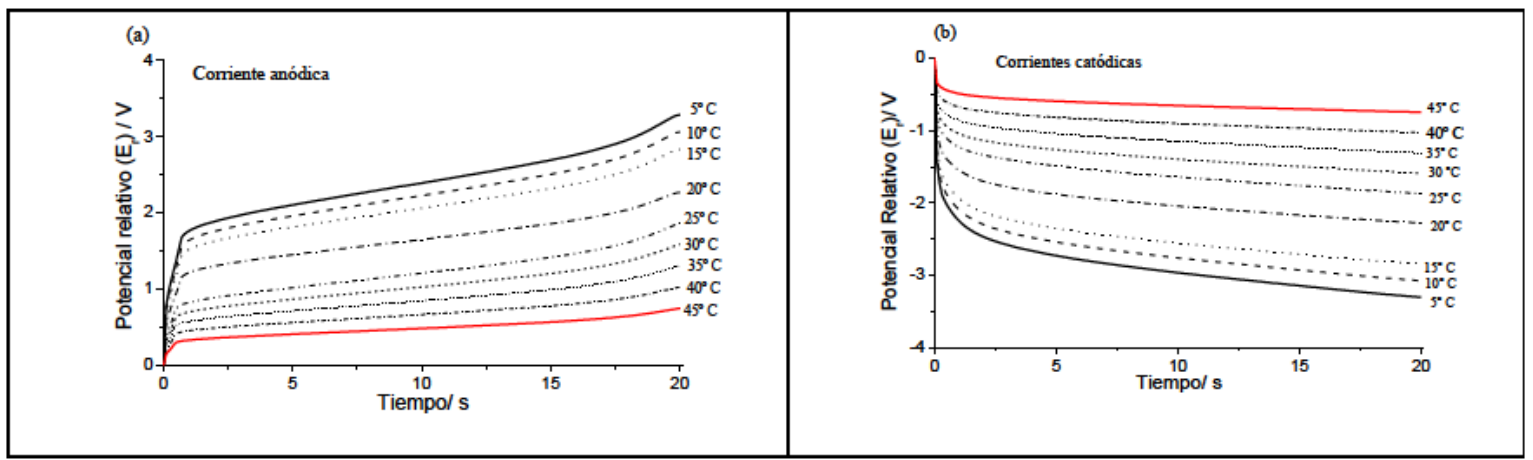

Figura 3.36. Respuesta anódica (a) y catódica (b) cronopotenciométrica de una tricapa pPy/DBS//cinta//pPy/DBS moviéndose al aplicar una onda cuadrada de corriente de $\pm 3 \mathrm{~mA}$ durante $20 \mathrm{~s}$ para obtener movimientos angulares de $\pm 45^{\circ}$ en una disolución acuosa $0.1 \mathrm{M}$ $\mathrm{LiClO}_{4}$ a diferentes temperaturas.

Por integración de las Figuras 3.35 (a) y 3.35 (b), se obtiene la energía eléctrica consumida a cada temperatura. La Figura 3.37 muestra un aumento lineal de la energía eléctrica consumida para describir un movimiento angular de $45^{\circ}$, cuando la temperatura disminuye: en presencia de menor energía térmica se consume mayor energía eléctrica y el actuador es un sensor de las condiciones térmicas de trabajo. Dicha relación lineal de la energía eléctrica consumida respecto a la temperatura puede es la curva de calibración de un sensor de temperatura siendo la pendiente (sensibilidad del sensor) de $0.5248 \mathrm{~J} /\left(\mathrm{g}^{\circ} \mathrm{C}\right)$ y $-0.4211 \mathrm{~J} /\left(\mathrm{g}^{\circ} \mathrm{C}\right)$ durante el paso de corrientes anódicas y catódicas, respectivamente.

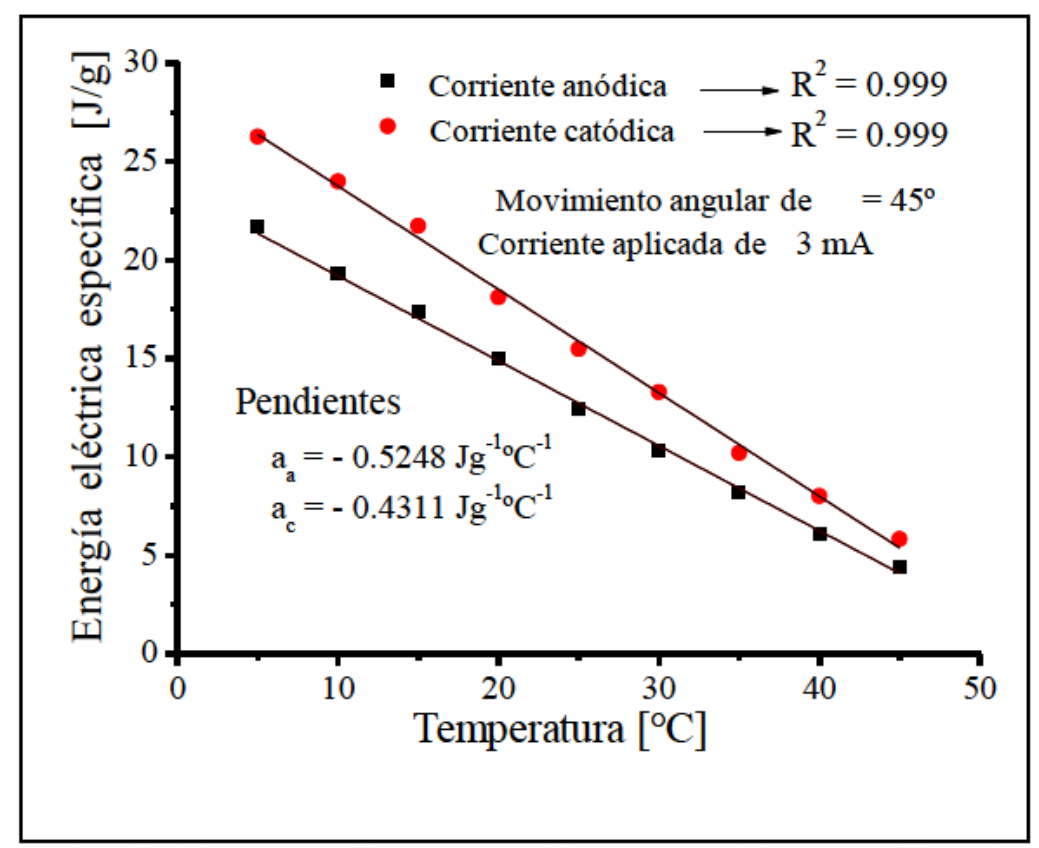

Figura 3.37. Energía eléctrica específica consumida por una tricapa a diferentes temperaturas durante los experimentos de la Figura 3.35 (a) y 3.35 (b). $R^{2}$ es el coeficiente de correlación del ajuste lineal; a es la sensibilidad de los sensores anódicos (subíndice a) y catódicos (subíndice c); $\theta$ es el ángulo desplazado. 
III.12.2.3 Influencia de Concentración del electrolito en el movimiento del actuador tricapa pPy/DBS//cinta//pPy/DBS

Por último se ha comprobado si este dispositivo tricapa es capaz de sentir, además, la concentración de electrolito en la que se encuentra inmerso, de forma similar a como ocurre con el material y el dispositivo bicapa. Para ello se procedió de forma similar a los casos anteriores, manteniendo constantes todas las condiciones experimentales excepto la concentración de electrolito, que se varió para cada experimento. Se estudiaron las respuestas cronopotenciométricas para diferentes concentraciones de electrolitos en disoluciones acuosas $\mathrm{LiClO}_{4}$, de $0.001 \mathrm{M}$ a $1 \mathrm{M}$, a $22^{\circ} \mathrm{C}$, mediante ondas cuadradas de corrientes constantes de $\pm 3 \mathrm{~mA}$, para describir una amplitud constante del movimiento angular de $\pm 45^{\circ}$. Se puede esperar de acuerdo a la reacción (1.2) que el aumento de las concentraciones en el electrolito (uno de los reactivos de dicha reacción) facilitará la reacción. En la Figura 3.38 se observa que la evolución del potencial muscular durante la actuación tiene lugar a potenciales menores cuando la concentración del electrolito aumenta.

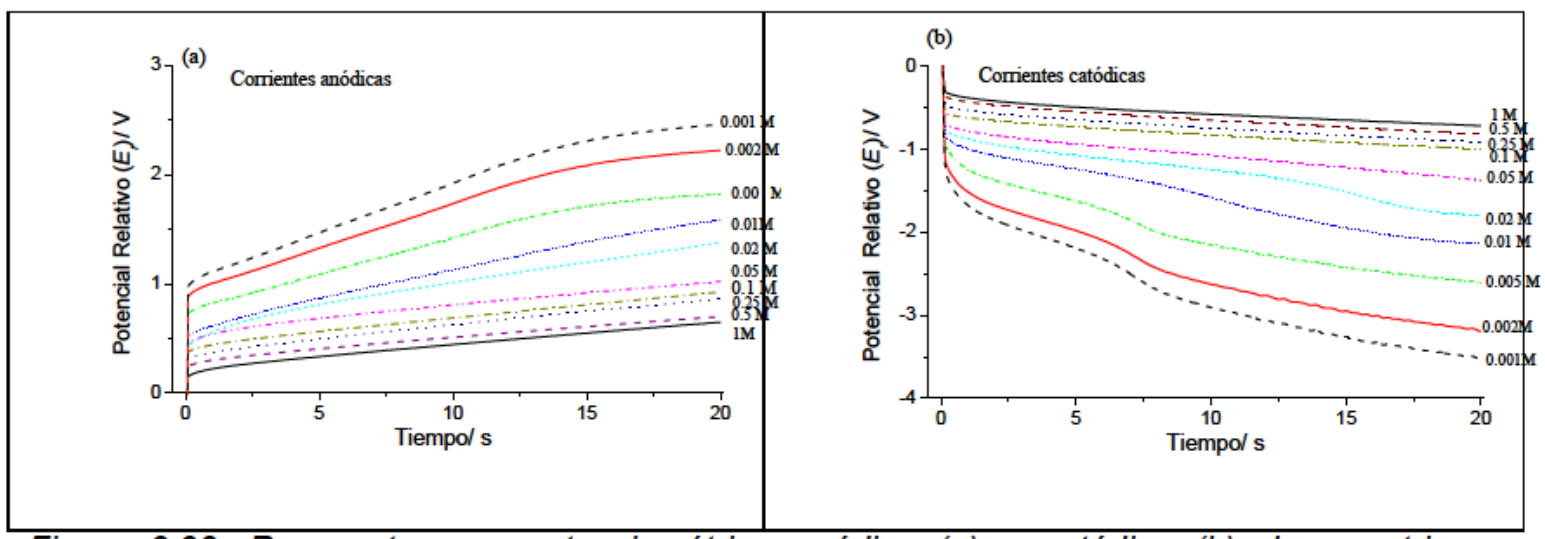

Figura 3.38. Respuesta cronopotenciométrica anódica (a) y catódica (b) de una tricapa pPy/DBS//cinta//pPy/DBS obtenida aplicando un flujo de corriente de $\pm 3 \mathrm{~mA}$ para obtener movimientos angulares $\pm 45^{\circ}$ en diferentes concentraciones de una disolución acuosa de $\mathrm{LiClO}_{4}$. a temperatura ambiente.

En la Figura 3.39 se muestra la variación de la energía eléctrica específica (obtenida por la integración de las respuestas de las Figuras 3.37 a y 3.37 b). La energía eléctrica consumida para describir un ángulo constante, aumenta al disminuir la concentración del electrolito siguiendo una relación doble logarítmica: con mayor energía química disponible (mayor potencial químico de los reactivos) se requiere el consumo de menor energía eléctrica para la actuación del dispositivo. Así, el actuador de triple capa es un sensor doble logarítmico de concentración mientras se está moviendo. La pendiente (sensibilidad del sensor) resulta ser de $-0.2328\left(\mathrm{Jg}^{-1}\right)[(\mathrm{M})]^{-1} \mathrm{y}-$ $0.2283\left(\mathrm{Jg}^{-1}\right)\left[(\mathrm{M})^{-1}\right]$. 


\section{Capítulo III}

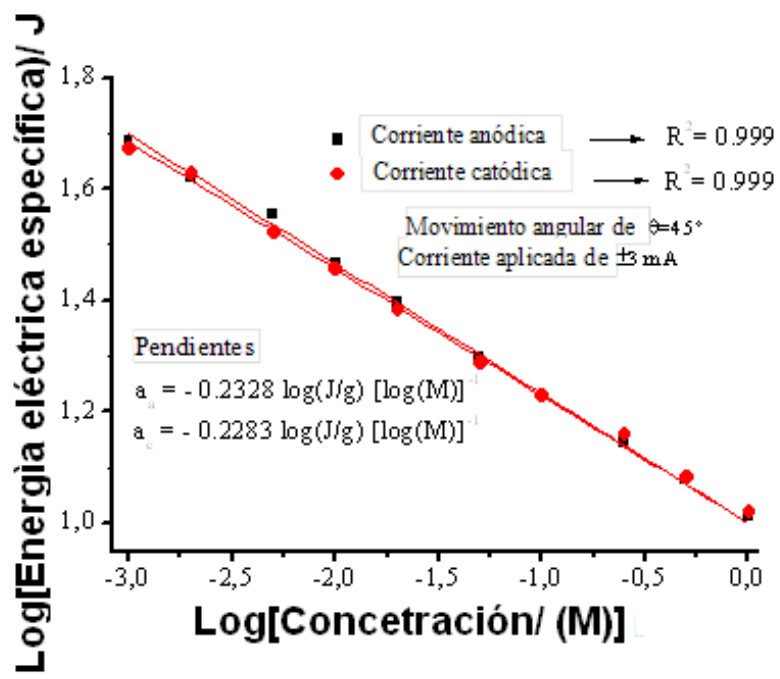

Figura 3.39. Variación de las energías eléctricas específicas (obtenido por la integración de las respuestas actuales correspondientes a las Figuras 3.37 a y 10 b) con el logaritmo de la concentración experimental del electrolito. $R^{2}$ es el coeficiente de correlación del ajuste lineal. a es la sensibilidad de los sensores anódicos (subíndice a) y catódicos (subíndice c); $\theta$ es el ángulo desplazado. 
Durante la realización de esta tesis se ha estudiado el comportamiento de un material (pPy/DBS), ya conocido por sus excelentes propiedades mecánicas pero todavía con muchas incógnitas desde el punto de vista de su comportamiento electro-quimio-mecánico, lo que limita su utilización en sistemas actuadores poliméricos robustos. Con el fin de clarificar dichos aspectos básicos de su funcionamiento, durante la realización de esta tesis se han ido llevando a cabo una serie de hitos:

Se han generado, por electropolimerizacion, películas poliméricas gruesas (varias decenas de micras) de base pPy, dopada con aniones de ácido dodecilbenzenosulfónico.

Las películas se separaron del electrodo de trabajo para ser empleadas como electrodos autosostenidos (películas autosoportadas), compuestas únicamente de polímero conductor.

Como electrodo autosoportado es un material blando (gel) cuyo contenido (polímero-ionesagua) mimetiza, en su expresión más simple) la matriz intracelular de las células vivas.

El desarrollo de modelos que expliquen su comportamiento del material o de los dispositivos construidos con el material, podrían servir de base para explicar los procesos que ocurren en el interior de la células de los seres vivos.

Las reacciones de oxidación-reducción en las películas de pPy/DBS utilizadas presentan en cualquier caso un consumo reversible de carga y otro consumo de carga irreversible a potenciales catódicos. Las respuestas culovoltamétricas permiten la determinación cuantitativa de las respectivas cargas. Dichas cargas reversibles o irreversibles están relacionadas con la oxidación/reducción del polímero conductor y con la evolución de hidrógeno desde el HDBS del polímero, respectivamente.

Durante la oxidación-reducción el culovoltagrama permite separar cinco cambios estructurales en la película controlados por las reacciones: oxidación-contracción, oxidación compactación, reducción-relajación, reducción hinchado y reducción con posible formación de vesículas.

El potencial del material y la energía consumida durante la reacción son funciones de las condiciones energéticas de trabajo (energía térmica, energía química o energía eléctrica). Durante la reacción el material siente las condiciones energéticas de trabajo y cualquier variación en dichas condiciones energéticas, pudiendo desarrollarse sensores que detectan dichos cambios: un sensor lineal de la corriente aplicada, un sensor lineal de la temperatura o un sensor semilogarítmico de la concentración del electrolito.

Los cambios reversibles de volumen producidos en la película por las reacciones reversibles fueron empleados en bicapas (pPy/DBS//cinta) y tricapas (pPy/DBS//cinta//pPy/DBS) para generar movimientos macroscópicos reversibles de flexión de los dispositivos: músculos artificiales. 
El sentido del movimiento ratifica que el movimiento está controlado por la reacción (1.2) en todo el rango de potenciales estudiados: el polímero conductor intercambia cationes durante las reacciones electroquímicas contrayéndose durante la oxidación por expulsión de cationes e hinchándose durante la reducción con entrada de cationes en el material.

La velocidad del movimiento angular de los músculos es una función lineal de la corriente eléctrica aplicada: el dispositivo es farádico y está controlado por la reacción (1.2). La constante de proporcionalidad es la constante amperodinámica del sistema.

Por su parte, el desplazamiento angular está controlado por la carga consumida: cuanto mayor sea la carga consumida mayor es el desplazamiento obtenido, observándose una relación lineal entre desplazamiento y carga. La constante de proporcionalidad es la constante culodinámica del sistema.

Ambos músculos artificiales (bicapa y tricapa) presentan propiedades similares a las películas autosoportadas. Son, simultáneamente, actuadores y sensores: de la corriente, de la concentración y de la temperatura, obteniéndose rectas de calibrado del sensor similares en cada caso.

El empleo de músculos artificiales en disoluciones del mismo anión y distintos cationes permite cuantificar otro aspecto básico de su funcionamiento: el intercambio de disolvente durante la reacción obteniéndose el número relativo de moléculas de agua que se intercambia con cada catión durante la reacción.

Se intentó ,además, buscar el origen de uno de los mayores inconvenientes que presentan este tipo de materiales para actuadores: el creeping, deslizamiento progresivo con el ciclado de su amplitud de desplazamiento por ciclo. De las respuestas culodinámicas de los músculos se deduce que este "creeping" es de origen electroquímico por las reacciones asociadas a las cargas irreversible durante la actuación. 
1. M. Alan G, Synthetic metals: a novel rule for organic polymers, Synthetic Metals 125, 11-22, 2002.

2. L. Stryer, J.M. Berg, J.L. Tymoczko, Biochemestry 4 th ed. W. H. Freeman \& Company, 1995.

3. Bar-Cohen Y., "Electroactive Polymer (EAP) Actuators as Artificial Muscles Reality, Potential and Challenges," 2nd Edition, ISBN 0-8194-5297-1, SPIE Press, Vol.136, 1-765, 2004.

4. Y. Osada, J.P. Gong, Nano-biomachine from actin and myosin gels, Polymer Science Series A 51, 689-700, 2009.

5. L. Valero, J. Arias-Padilla, J. Cauich-Rodriguez, M. Smit, T.F. Otero, Sensing and tactile artificial muscles from reactive materials, Sensors 10, 1-39, 2010.

6. T.F. Otero, M.T. Cortes, A sensing muscle, Sensors and Actuators B: Chemical 96, 152-156, 2003.

7. W. Kuhn, B. Hargitay, A. Katchalsky, H. Eisenberg, Reversible Dilation and Contraction by Changing the State of Ionization of High-Polymer Acid Networks, Nature 165, 514-516, 1950.

8. T. Kurauchi, T. Shiga, Y. Hirose, A. Okada, Deformation-Behavior of Polymer Gels in Electric-Field, Polymer Based Molecular Composites. Materials research society symposium proceedings 1, 389-394, 1990.

9. T.F. Otero, J. Rodriguez en Intrinsically conducting polymers: An emerging technology. Aldissi, M., Ed.; Kluwer, Dordrecht (The Nederlands), 179-190, 1993.

10. Y. Bar-Cohen, S. Leary, M. Shahinpoor, J.O. Harrison, J. Smith, Electro-Active Polymer (EAP) actuators for planetary applications, Proceedings of SPIE's EAPAD Conference 3669, 57-63, 1999.

11. Y. Fang, X.O. Tan, Y.T. Shen, N. Xi, G. Alici, A scalable model for trilayer conjugated polymer actuators and its experimental validation, Materials Science \& Engineering C-Biomimetic and Supramolecular Systems 28, 421-428, 2008.

12. T.F. Otero, Reactions drive conformations. Biomimetic properties and devices, theoretical description, Journal of Materials Chemistry B, 1, 3754-3767, 2013.

13. M.S. Freund, B. Deore Self-Doped Conducting Polymers, John Wiley \& Sons, Chichester, 2007.

14. T.F.Otero, H.Grande Handbook of Conducting Polymers, T. Skotheim, J. Reynolds. CRC Press, 541-624, 2006.

15. T. Fernandez-Otero, H.J. Grande, Electromechanical Devices: Artificial Muscles Based on Conducting Polymers, Universidad del Pais Vasco, San sebastian, EspañaBioelectrochem Bioenergy 38411, 1998. 
16. Dyer John.R., Aplications of absorption Spectroscopy of organic compounds, Prentice Hall INC, Englewood Cliffs, N.J., 1965.

17. T. Fernandez-Otero, Materiales inteligentes Realidad y perpectivas de futuro en domotica, Medio ambiente, 2002.

18. T.F. Otero en Modern aspects of electrochemistry, Bockris, J. O'M., White, R. E., and Conway, B. E., Ed.; Kluwer Academic/Plenum Publ, New York, 307-434, 1999.

19. H. Shirakawa, E.J. Louis, A.G. Macdiarmid, C.K. Chiang, A.J. Heeger, Synthesis of Electrically Conducting Organic Polymers - Halogen Derivatives of Polyacetylene, (Ch)X, Journal of the Chemical Society-Chemical Communications, 578-580, 1977.

20. T. Yamamoto, K. Sanechika, A. Yamamoto, Preparation of thermostable and electric-conducting poly(2,5-thienylene), Journal of Polymer Science: Polymer Letters Edition 18, 9-12, 1980.

21. W.Gopel, A gas sensor with porous films of Bi2Sr2CaCu2O8: an analysis of the response, Sensors and Actuators B:Chemical, 22, 227-232, 1994.

22. D. Zhou, G.M. Spinks, G.G. Wallace, C. Tiyapiboonchaiya, D.R. MacFarlane, M. Forsyth, J. Sun, Solid state actuators based on polypyrrole and polymer-in-ionic liquid electrolytes, Electrochimica Acta 48, 2355-2359, 2003.

23. W. Göpel, H.J., J.N. Zemel, Controlled signal transduction across interfaces of "intelligent" molecular systems, Biosensors and Bioelectronics 10, 35-39, 1995.

24. T.F. Otero, Biomimetic Conducting Polymers: Synthesis, Materials, Properties, Functions, and Devices, Polymer Reviews 53, 311-351, 2013.

25. A. Dallolio, G. Dascola, V. Varacca, V. Bocchi, Electronic Paramagnetic Resonance and Conductivity of A Black Electrolytic Oxypyrrole, Comptes Rendus Hebdomadaires des Seances de I Academie des Sciences Serie C 267, 433,1968.

26. A.F. Diaz, K.K. Kanazawa, G.P. Gardini, Electrochemical Polymerization of Pyrrole, Journal of the Chemical Society-Chemical Communications, 635-636, 1979.

27. A.F. Diaz, W.Y. Lee, A. Logan, D.C. Green, Chemical modification of a polypyrrole electrode surface, Journal of Electroanalytical Chemistry 108, 377-380, 1980.

28. S. Rapi, V. Bocchi, G.P. Gardini, Conducting polypyrrole by chemical synthesis in water, Synthetic. Metals. 24, 217-221, 1988.

29. J. Rodriguez, H.J. Grande, T.F. Otero en Handbook of Organic Conductive Molecules and Polymers, Nalwa, H. S., Ed.; John Wiley \& Sons, Chichester, 415-468, 1997.

30. M. Salmon, K.K. Kanazawa, A.F. Diaz, M. Krounbi, A Chemical Route to Pyrrole Polymer-Films, Journal of Polymer Science Part C-Polymer Letters 20, 187-193, 1982. 
31. G.G. Wallace, G.M. Spinks, L.A.P. Kane-Maguire, P.R. Teasdale en Conductive electroactive polymers: Intelligent materials systemsAssembly of polypyrroles, CRC Press, Boca Raton, 51-88, 2003.

32. W.S. Huang, B.D. Humphrey, A.G. Macdiarmid, Polyaniline, A Novel Conducting Polymer - Morphology and Chemistry of Its Oxidation and Reduction in Aqueous-Electrolytes, Journal of the Chemical Society-Faraday Transactions I 82, 2385-1986.

33. A. Kitani, M. Kaya, K. Sasaki, Performance Study of Aqueous Polyaniline Batteries, Journal of the Electrochemical Society 133, 1069-1073, 1986.

34. $H$. Letheby, XXIX.On the production of a blue substance by the electrolysis of sulphate of aniline, Journal of the Chemical Society 15, 161-163, 1862.

35. D.M. Mohilner, W.J. Argersinger, R.N. Adams, Investigation of Kinetics and Mechanism of Anodic Oxidation of Aniline in Aqueous Sulfuric Acid Solution at A Platinum Electrode, Journal of the American Chemical Society 84, 3618-1962.

36. D.C. Trivedi en Handbook of Organic Conductive Molecules and Polymers, Nalwa, H. S., Ed.; John Wiley \& Sons, Chichester, 505-572, 1997.

37. G.G. Wallace, G.M. Spinks, L.A.P. Kane-Maguire, P.R. Teasdale en Conductive Electroactive Polymers: Intelligent Materials Systems, CRC Press, Boca Raton, 179206, 2003.

38. T. Anjos, A. Charlton, S.J. Coles, A.K. Croft, M.B. Hursthouse, M. Kalaji, P.J. Murphy, S.J. Roberts-Bleming, Electropolymerization Studies on a Series of Thiophene-Substituted 1,3-Dithiole-2-ones: Solid-State Preparation of a Novel TTFDerivatized Polythiophene, Macromolecules 42, 2505-2515, 2009.

39. M.A. Del Valle, M.A. Gacitua, L.I. Canales, F.R. Diaz, Oligomer Chain Length Effect on the Nucleation and Growth Mechanisms (Ngm) of Polythiophene, Journal of the Chilean Chemical Society 54, 260-266, 2009.

40. M.S. Freund, B. DeoreSelf-Doped Conducting Polymers, John Wiley \& Sons, Chichester, 2007.

41. G. Tourillon, F. Garnier, New electrochemically generated organic conducting polymers, Journal of Electroanalytical Chemistry 135, 173-178, 1982.

42. G. Zotti, G. Schiavon, N. Comisso, A. Berlin, G. Pagani, Electrochemical synthesis and characterization of polyconjugated polyfuran, Synthetic Metals 36, 337$351,1990$.

43. A.F. Diaz, J. Castillo, K.K. Kanazawa, J.A. Logan, M. Salmon, O. Fajardo, Conducting poly-N-alkylpyrrole polymer films, Journal of Electroanalytical Chemistry 133, 233-239, 1982.

44. D. Macinnes, B.L. Funt, Poly-Ortho-Methoxyaniline - A New Soluble Conducting Polymer, Synthetic Metals 25, 235-242, 1988. 
45. N. Somanathan, S. Radhakrishnan, Optical properties of functionalized polythiophenes, International Journal of Modern Physics B 19, 4645-4676, 2005.

46. R.J. Waltman, A.F. Diaz, J. Bargon, Substituent Effects in the Electropolymerization of Aromatic Heterocyclic-Compounds, Journal of the Physical Chemistry 88, 4343-4346, 1984.

47. A.Malinauskas, Self doped polyanilines, Journal of the Power Sources 126(1-2), 214-220, 2004.

48. M.S.A. Abdou, S. Holdcroft en Handbook of Organic Conductive Molecules and Polymers, Nalwa, H. S., Ed.; John Wiley \& Sons, Chichester, 833-858, 1997.

49. K.P.a.A.Y. Y.A.Udum, Electropolymerization of self-doped polythiopehene in acetonitrile containing FSO3H, Synthetic Metals 142(1-3), 7-12, 2004.

50. J. Carrasco, A. Figueras, T.F. Otero, E. Brillas, Anodic electrosynthesis and cathodic electrodissolution of poly(2,5-di-(2-thienyl)pyrrole). A new way of processibility, Synthetic Metals 61, 253-258, 1993.

51. J.P. Ferraris, D.J. Guerrero en Handbook of Conducting Polymers, Skotheim, T., Elsenbaumer, R. L., and Reynolds, J. R., Ed.; Marcel Dekker, New York, 259-276, 1997.

52. R.W. Gumbs en Handbook of Organic Conductive Molecules and Polymers, Nalwa, H. S., Ed.; John Wiley \& Sons, Chichester, 469-504, 1997.

53. A.I. Nazzal, G.B. Street, Pyrrole Styrene Graft-Copolymers, Journal of the Chemical Society-Chemical Communications, 375-376, 1985.

54. Y. Wei, R. Hariharan, S.A. Patel, Chemical and Electrochemical Copolymerization of Aniline with Alkyl Ring-Substituted Anilines, Macromolecules 23, 758-764, 1990.

55. M. Clemente-Leon, E. Coronado, J.R. Galan-Mascaros, C. Gimenez-Saiz, C.J. Gomez-Garcia, T. Fernandez-Otero, Hybrid molecular materials based on organic molecules and the inorganic magnetic cluster [M-4(H2O)(2)(PW9O34)(2)](10-) (M2+ = Co, Mn), Journal of the Materials Chemistry 8, 309-312, 1998.

56. P. Gómez-Romero, M. Lira-Cantú, Hybrid organic-inorganic electrodes: The molecular material formed between polypyrrole and the phosphomolybdate anion, Advanced Materials 9, 144-147, 1997.

57. Saunders R, K.S. Murray, R. Cervini, N.S. Allen en Handbook of Organic Conductive Molecules and Polymers. 3rd ed. Nalwa H.S., editor. John Wiley \& Sons; Chichester, UK, 634-676, 1997.

58. T.F. Otero, M.V. Vázquez, Electrogeneration of a composite polypyrrolecarboxymethylcellulose: kinetic study, Journal of Electroanalytical Chemistry 397, 171176, 1995. 
59. T.F. Otero, V. Olazábal, Electrogeneration of polypyrrole in presence of polyvinylsulphonate. Kinetic study, Electrochimica Acta 41, 213-220, 1996.

60. T.F. Otero, J.M. Sansiñena, Influence of synthesis conditions on polypyrrolepoly(styrenesulphonate) composite electroactivity, Journal of Electroanalytical Chemistry 412, 109-116, 1996.

61. T.F. Otero, M.J. González-Tejera, Polypyrrole + polyacrylate composites: electrogeneration, Journal of Electroanalytical Chemistry 410, 69-77, 1996.

62. M.A. De Paoli en Handbook of Organic Conductive Molecules and Polymers, Nalwa, H. S., Ed.; John Wiley \& Sons, Chichester, 773-798, 1997.

63. J.C. Huang, Carbon black filled conducting polymers and polymer blends, Advances in Polymer Technology 21, 299-313, 2002.

64. T.F. Otero, M.J. Gonzalez Tejera, Polypyrrole plus polyacrylate composites, kinetic study, Journal of Electroanalytical Chemistry, 412(1-2).109-116, 1996.

65. T.F. Otero, P. Herrasti, P. Ocon, C.R. Alves, Electrogeneration of polypyrrolecarboxymethylcellulose composites: electrochemical, microgravimetric and morphological studies, Electrochimica Acta 43, 1089-1100, 1998.

66. E. Ruckenstein, J.H. Chen, Polypyrrole Conductive Composites Prepared by Coprecipitation, Polymer 32, 1230-1235, 1991.

67. D. Braun and H.Cherdron, Polymer Synthesis: Theory and Practice: Fundamentals, Methods, Experiments 5th ed. Springer, 2013

68. S. Sadki, P. Schottland, N. Brodie, G. Sabouraud, The mechanisms of pyrrole electropolymerization, Chemical Society Reviews 29, 283-293, 2000.

69. T. V. Vernitskaya and O. N. Efimov, Polypyrrole: a conducting polymer; its synthesis, properties and applications Russian Chemical Reviews 66, 443-457, 1997.

70. T. Zama, S. Hara, W. Takashima and K. Kaneto, Comparison of conducting polymer actuators based on polypyrrole doped with BF4(-), PF6(-), CF3SO3-, and ClO4 Bulletin of the Chemical Society of Japan 78, 506-511, 2005.

71. S. Hara, T. Zama, W. Takashima and K. Kaneto, Artificial muscles based on polypyrrole actuators with large strain and stress induced electrically Polymer Journal 36, 151-161, 2004.

72. L. Viau, J. Y. Hihn, S. Lakard, V. Moutarlier, V. Flaud and B. Lakard, Full characterization of polypyrrole thin films electrosynthesized in room temperature ionic liquids, water or acetonitrile, Electrochimica Acta 137, 298-310, 2014.

73. M. Pyo, J. Reynolds, L. Warren and H. Marcy, LONG-TERM REDOX SWITCHING STABILITY OF POLYPYRROLE Synthetic Metals, 68, 71-77, 1994. 
74. T. F. Otero and I. Cantero, Statistical design to optimize specific charges in polypyrrole by electrosynthesis Journal of the Electrochemical Society 146, 41184123, 1999.

75. T. Otero and J. Rodriguez, Parallel kinetic studies of the electrogeneration of conducting polymers: mixed materials, composition and properties control Electrochimica Acta 39, 245-253, 1994.

76. T.F. Otero, I. Boyano, Nucleation and nonstoichiometry in electrochromic conducting polymers, Chemphyschem 4, 868-872, 2003.

77. Steen Skaarup, Lasse Bay, Kamal Vidanapathirana, Susanne Thybo, Pentti Tofte, Keld Wes, Simultaneous anion and cation mobility polypyrrole, Solid State lonics: Proceedings 159, 143-147, 2003.

78. T.F.Otero, Handbook of Organic Conductive Molecules and Polymers, Volume 4, Conductive Polymers: Transport, Photophysics and Applications, H. S. Nalwa, editor, J. Wiley \& Sons, Chichester UK, 1997.

79. Ansari R.Klalkhali, A. Tamm J, Hallik A.Johansan, Tamm L, Electrochemical Synthesis and Characterization of electroactive conducting Polypyrrole Polymers, Russian Journal of Electrochemestry 41, 950-955, 2005.

80. Bar Yosph CohenElectroactive Polymers (EAP) Actuators as Artificial Muscles, SPIE press, Washington, 2ed., 2004.

81. R.H. Baughman, L.W. Shacklette en Science and Application of Conducting Polymers, Salaneck, W. R., Clark, D. T., and Samuelsen, E. J., Ed.; IOP Publishing Ltd, Bristol, 47-61, 1991.

82. R.H. Baughman, Conducting polymer artificial muscles, Synthetic Metals 78, 339-353, 1996.

83. W.S. Huang, B.D. Humphrey, A.G. Macdiarmid, Polyaniline, A Novel Conducting Polymer - Morphology and Chemistry of Its Oxidation and Reduction in Aqueous-Electrolytes, Journal of the Chemical Society-Faraday Transactions I 82, 2385, 1986.

84. T. Iyoda, A. Ohtani, T. Shimidzu, K. Honda, Conducting Polymer Electrodes with High Performances Through Anodic Doping Process, Synthetic Metals 18, 725730, 1987.

85. F.B. Li, W.J. Albery, Electrochemical and Insitu Electron-Paramagnetic Resonance Studies of Polypyrrole Doped with Polystyrene Sulfonate, Journal of the Chemical Society-Faraday Transactions 87, 2949-2954, 1991.

86. T. Shimidzu, A. Ohtani, T. lyoda, K. Honda, Effective Adsorption Desorption of Cations on A Polypyrrole Polymer Anion Composite Electrode, Journal of the Chemical Society-Chemical Communications, 1415-1417, 1986.

87. T. Shimidzu, A. Ohtani, T. Iyoda, K. Honda, Charge-Controllable Polypyrrole Polyelectrolyte Composite Membranes .2. Effect of Incorporated Anion Size on the 
Electrochemical Oxidation Reduction Process, Journal of Electroanalytical Chemistry 224, 123-135, 1987.

88. J. Yue, Z.H. Wang, K.R. Cromack, A.J. Epstein, A.G. Macdiarmid, Effect of Sulfonic-Acid Group on Polyaniline Backbone, Journal of the American Chemical Society 113, 2665-2671, 1991.

89. A.L. Dyer, J.R. Reynolds en Handbook of Conducting Polymers, Skotheim, T. A., Elsenbaumer, R. L., and Reynolds, J. R., Ed.; CRC Press, Boca Raton, 1-63, 2007.

90. H.W. Heuer, R. Wehrmann, S. Kirchmeyer, Electrochromic window based on conducting poly (3,4-ethylenedioxythiophene)poly(styrene sulfonate), Advanced Functional Materials 12, 89-94, 2002.

91. J.H. Kaufman, K.K. Kanazawa, G.B. Street, Gravimetric Electrochemical Voltage Spectroscopy - Insitu Mass Measurements During Electrochemical Doping of the Conducting Polymer Polypyrrole, Physical Review Letters 53, 2461-2464, 1984.

92. R.J. Mortimer, A.L. Dyer, J.R. Reynolds, Electrochromic organic and polymeric materials for display applications, Displays 27, 2-18, 2006.

93. V. Seshadri, J. Padilla, H. Bircan, B. Radmard, R. Draper, M. Wood, T.F. Otero, G.A. Sotzing, Optimization, preparation, and electrical short evaluation for $30 \mathrm{~cm}(2)$ active area dual conjugated polymer electrochromic windows, Organic Electronics 8, 367-381, 2007.

94. D.Pacheco Catalán, E.Morales, M.Smit, J.L.Acosta, Electrocatalytic activity towards oxygen reduction of mesoporous carbon/conducting polymer composites Application to PEM fuel cells, Journal of New Materials for Electrochemical Systems 12, 115-122, 2011.

95. P.Novak, K.Muller, K.S.V.Santhanam, O.Hass, Electrochemically active polymers for rechargable batteries, Chemical Reviews 97(1), 207-281, 1997.

96. Yun Hui Huang, John B.Goodenough, High-Rate LiFePO4 Lithium Rechargeable Battery Promoted by Electrochemically Active Polymers, Chemistry of materials 20, 7237-7241, 2008.

97. T.F. Otero, S.O. Costa, M.J. Ariza, M. Marquez, Electrodeposition of $\mathrm{Cu}$ on deeply reduced polypyrrole electrodes at very high cathodic potentials, Journal of Materials Chemistry 15, 1662-1667, 2005.

98. T.F. Otero, M.J. Ariza, Revisiting the electrochemical and polymeric behavior of a polypyrrole free-standing electrode in aqueous solution, Journal of Physical Chemistry B 107, 13954-13961, 2003.

99. P. Burgmayer, R.W. Murray, An Ion Gate Membrane - Electrochemical Control of Ion Permeability Through A Membrane with An Embedded Electrode, Journal of the American Chemical Society 104, 6139-6140, 1982. 
100. C. Chan, S. Chang, H.E. Naguib, Development and characterization of polypyrrole bi-layer and tri-layer thin porous films, Smart Materials \& Structures 18, 2009.

101. C. Ehrenbeck, K. Juttner, Development of an anion cation permeable freestanding membrane based on electrochemical switching of polypyrrole, Electrochimica Acta 41, 511-518, 1996.

102. C. Ehrenbeck, K. Juttner, lon conductivity and permselectivity measurements of polypyrrole membranes at variable states of oxidation, Electrochimica Acta 41, 18151823, 1996.

103. T.F. Otero, Biomimetics, Artificial Muscles \&amp; Nano-Bio 2007: Scientists Meet Doctors, Journal of Physics: Conference Series 127, 011001, 2008.

104. Y. Zhong, R.V. Bellamkonda, Controlled release of anti-inflammatory agent [alpha]-MSH from neural implants, Journal of Controlled Release 106, 309-318, 2005.

105. L.L. Miller, B. Zinger, Q.X. Zhou, Electrically controlled release of hexacyanoferrate(4-) from polypyrrole, Journal of the American Chemical Society 109, 2267-2272, 1987.

106. B. Zinger, L.L. Miller, Timed release of chemicals from polypyrrole films, Journal of the American Chemical Society 106, 6861-6863, 1984.

107. T.F. Otero, Biomimicking materials with smart polymers, in: M. Elices, R.W. Cahn (Eds.), Struct. Biol. Mater. Des. Struct.-Prop. Relatsh., Pergamon, Amsterdam, 2000.

108. T.F. Otero, M.T. Cortes, Artificial muscle: movement and position control, Chemical Communications, 284-285, 2004.

109. Cortes Maria Teresa, J.Carlos Moreno, Artificial muscles based on conducting polymers, e-polymers 41, 1-42, 2003

110. E. Smela, Conjugated polymer actuators for biomedical applications, Advanced Materials 15, 481-494, 2003.

111. E.W.H.Jager, Elisabeth Smela, le Inganas, Microfabricating Conjugated Polymer Actuators, Science 24, 1540-1545, 2000.

112. T. F. Otero Conducting polymers, electrochemistry and biomimicking processes. In: RE White, JOM Bockris, BE Conway, editors. Modern Aspects of Electrochemistry, vol. 33. New York: Springer US; 307-434, 1999.

113. T.F. Otero, Soft, wet, and reactive polymers. Sensing artificial muscles and conformational energy, Journal of Materials Chemistry 19, 681-689, 2009.

114. T.F. Otero en Handbook of conducting polymers, Skotheim, T. A., Elsenhaumer, R. L., and Reynolds, J. R., Ed.; Marcel Dekker Inc., New York, 10151028, 1998. 
115. Cortes Maria Teresa, "Artificial muscles base on conducting polymers"; epolymers 119-126, 2003.

116. Toribio F. Otero; Jose G. Martinez, Reactive actuators and sensors integrated in one device: mimicking brain-muscles feedback communication, Proc. SPIE 8687, Electroactive Polymer Actuators and Devices, 8687, 10-18, 2013.

117. T.F. Otero, M.T. Cortes, Artificial muscles with tactile sensitivity, Advanced Materials 15, 279-282, 2003.

118. Cortes Maria Teresa, J.Carlos Moreno, Artificial muscles based on conducting polymers, e-polymers 41, 1-42, 2003.

119. T.F.Otero, G.Vázquez Arenas, J.J.López Cascales, Effect of the doping lon on the electrical response of a free standing polypyrrole strip subjected to different preloads: Perspectives and limitation associated with the use of these devices actuators, Macromolecules 39, 9551-9556, 2006.

120. T.F. Otero, J.J. Lopez Cascales, G. Vazquez Arenas, Mechanical characterization of free-standing polypyrrole film, Materials Science and Engineering: $\mathrm{C}$ 27, 18-22, 2007.

121. P.G.A. Madden, J.D.W. Madden, P.A. Anquetil, N.A. Vandesteeg, I.W. Hunter, The relation of conducting polymer actuator material properties to performance, IEEE Journal of Oceanic Engineering 29, 696-705, 2004.

122. A. DellaSanta, D. DeRossi, A. Mazzoldi, Performance and work capacity of a polypyrrole conducting polymer linear actuator, Synthetic Metals 90, 93-100, 1997.

123. S. Hara, T. Zama, S. Sewa, W. Takashima, K. Kaneto, Highly stretchable and powerful polypyrrole linear actuators, Chemistry Letters 32, 576-577, 2003.

124. J. Madden, I.W. Hunter, R.J. Gilbert, Development of an artificial muscle fiber composed of the conducting polymer actuator polypyrrole, Gastroenterology 122, S1111, 2002.

125. J.D.W. Madden, N.A. Vandesteeg, P.A. Anquetil, P.G.A. Madden, A. Takshi, R.Z. Pytel, S.R. Lafontaine, P.A. Wieringa, I.W. Hunter, Artificial muscle technology: Physical principles and naval prospects, leee Journal of Oceanic Engineering 29, 706728, 2004.

126. A. Mazzoldi, C. Degl'Innocenti, M. Michelucci, D. De Rossi, Actuative properties of polyaniline fibers under electrochemical stimulation, Materials Science \& Engineering C-Biomimetic Materials Sensors and Systems 6, 65-72, 1998.

127. T.F.Otero, M.Broschar, Polypyrrole artificial muscles: a new rhombic element. Construction and electromechanical characterization, Journal of Applied Electrochemistry 36, 205-214, 2006.

128. A. DellaSanta, D. DeRossi, A. Mazzoldi, Characterization and modelling of a conducting polymer muscle-like linear actuator, Smart Materials \& Structures 6, 23-34, 1997. 
129. J. Ding, L. Liu, G.M. Spinks, D. Zhou, G.G. Wallace, J. Gillespie, High performance conducting polymer actuators utilising a tubular geometry and helical wire interconnects, Synthetic Metals 138, 391-398, 2003.

130. S. Hara, T. Zama, W. Takashima, K. Kaneto, Polypyrrole-metal coil composite actuators as artificial muscle fibres, Synthetic Metals 146, 47-55, 2004.

131. Tina Shoa et al,Electromechanical coupling in polypyrrole sensors and actuators, Sensors and Actuators A Physical.; 161(1-2):127-133, 2010

132. T.F.Otero, M.T.Cortés, I.Boyano, Macroscopic devices and complex movements developed with artificial muscles, Proceedings of the Smart Structures and Materials, 2002.

133. K. Yamato, K. Kaneto, Tubular linear actuators using conducting polymer, polypyrrole, Analytica Chimica Acta 568, 133-137, 2006.

134. C. Chan, S. Chang, H.E. Naguib, Development and characterization of polypyrrole bi-layer and tri-layer thin porous films, Smart Materials \& Structures 18, 2009.

135. R. Minato, G. Alici, S. McGovern, G. Spinks, Tri-layer conducting polymer actuators with variable dimensions - art. no. 65241J, Electroactive Polymer Actuators and Devices (EAPAD) 6524, J5241-J5241, 2007.

136. G.Y. Han, G.Q. Shi, High-response tri-layer electrochemical actuators based on conducting polymer films, Journal of Electroanalytical Chemistry 569, 169-174, 2004.

137. A. Kaynak, C.H. Yang, Y.C. Lim, A. Kouzani, Electrochemical fabrication and modelling of mechanical behavior of a tri-layer polymer actuator, Materials Chemistry and Physics 125, 113-117, 2011.

138. T.F. Otero, J. Rodriguez, Kinetics of Polypyrrole Electrogeneration on $\mathrm{Pt}$ Through Tafel Slopes, Synthetic Metals 55, 1436-1440, 1993.

139. T.F. Otero, M.T. Cortes, Artificial muscle: movement and position control, Chemical Communications, 284-285, 2004.

140. T.F.Otero, H.Grande Handbook of Conducting Polymers, T. Skotheim, J. Reynolds. CRC Press, 541-624, 2006.

141. T.F.Otero, G.Vázquez, L.Valero, Conducting polymers as simultaneous sensoractuators, SPIE press, Electroactive Polymer Actuators and devices Vol. 7642-W1, 2010.

142. G.Y. Han, G.Q. Shi, High-response tri-layer electrochemical actuators based on conducting polymer films, Journal of Electroanalytical Chemistry 569, 169-174, 2004.

143. S. Hara, T. Zama, W. Takashima, K. Kaneto, TFSI-doped polypyrrole actuator with 26\% strain, Journal of Materials Chemistry 14, 1516-1517, 2004. 
144. M. Onoda, Y. Kato, H. Shonaka, K. Tada, Artificial muscle using conducting polymers, Electrical Engineering in Japan 149, 7-13, 2004.

145. G.Y. Han, G.Q. Shi, High-response tri-layer electrochemical actuators based on conducting polymer films, Journal of Electroanalytical Chemistry 569, 169-174, 2004.

146. G. Han, G. Shi, Conducting polymer electrochemical actuator made of highstrength three-layered composite films of polythiophene and polypyrrole, Sensors and Actuators B: Chemical 99, 525-531, 2004.

147. K. Kaneto, M. Kaneko, Y. Min, A.G. MacDiarmid, "Artificial muscle": Electromechanical actuators using polyaniline films, Synthetic Metals 71, 2211-2212, 1995.

148. H. Okuzaki, N. Shirai, Contractile behavior of polypyrrole films in air under ac voltages, Synthetic Metals 153, 109-112, 2005.

149. M. Onoda, K. Tada, Anisotropic bending machine using conducting polypyrrole, leice Transactions on Electronics E87C, 128-135, 2004.

150. T.F. Otero, E. Angulo, Impedance Studies of Electrogenerated Polypyrrole Films, Synthetic Metals 51, 87-94, 1992.

151. T.F.Otero, M.Broschar, Polypyrrole artificial muscles: a new rhombic element. Construction and electromechanical characterization, Journal of Applied Electrochemistry 36, 205-214, 2006.

152. J.M.Sansiñena, T.F.Otero, Artificial Muscles Based on Conducting Polymers, Bioelectrochemistry and Bioenergetics, 42-117, 1997.

153. M.T.Cortes. T.F.Otero, Proceedings of the Smart Structures and Materials 934329, 2001.

154. M. Onoda, Y. Kato, H. Shonaka, K. Tada, Artificial muscle using conducting polymers, Electrical Engineering in Japan 149, 7-13, 2004.

155. T. Fernandez-Otero, H.J. Grande, Electromechanical Devices: Artificial Muscles Based on Conducting Polymers, Bioelectrochemistry and Bioenergetics 38411, 1998.

156. T.F. Otero, Reactive conducting polymers as actuating sensors and tactile muscles, Bioinspiration \& Biomimetics 3, 1-9, 2008.

157. S. Hara, T. Zama, A. Ametani, W. Takashima, K. Kaneto, Enhancement in electrochemical strain of a polypyrrole-metal composite film actuator, Journal of Materials Chemistry 14, 2724-2725, 2004.

158. S. Hara, T. Zama, W. Takashima, K. Kaneto, Tris(trifluoromethylsulfonyl)methide-doped polypyrrole as a conducting polymer actuator with large electrochemical strain, Synthetic Metals 156, 351-355, 2006. 
159. T.F. Otero, E. Angulo, J. Rodriguez, C. Santamaria, Electrochemomechanical Properties from A Bilayer - Polypyrrole Nonconducting and Flexible Material Artificial Muscle, Journal of Electroanalytical Chemistry 341, 369-375, 1992.

160. Wen Zheng, Analysis of high permormance polypirrole actuators, University of wollongogmg,2011, (www.uow.edu.au/cgi).

161. Artur de Jesus Motheo, Aspects on fundaments and applications of conducting, Published by InTech. 2012

.162. Laura. L Valero,Toribio F. Otero; Jose G. Martinez,, Polymeric Artificial Muscles are Linear Faradaic Motors, Key Engineering Materials, Volu. 644, 2015

163. T.F. Otero, J.G. Martinez, Physical and chemical awareness from sensing polymeric artificial muscles. Experiments and modeling, Progress in Polymer Science 44, 62-78, 2015.

164. Toribio F. Otero, Jose G. Martinez, Can human proprioception be described by physical-chemical equations? Proprioceptive artificial muscles, Key Engineering Materials Vol 644, 145-152, 2015.

165. D. Svirskis, B.E. Wright, J. Travas-Sejdic, A. Rodgers, S. Garg, Evaluation of physical properties and performance over time of an actuating polypyrrole based drug delivery system Sensors and Actuators B: Chemical, 151 ,97, 2010.

166. P.M. George, D.A. Lavan, J.A. Burdick, C.Y. Chen, E. Liang, R. Langer, Electrically controlled drug delivery from biotin-doped conductive polypyrrole, Advanced Materials, 18, 577, 2010.

167. Y.L. Li, K.G. Neoh, E.T. Kang, Controlled release of heparin from polypyrrolepoly(vinyl alcohol) assembly by electrical stimulation Journal of Biomedical Materials Research Part A, 73A ,171, 2005.

168. X.L. Luo, X.T. Cui, Sponge-like nanostructured conducting polymers for electrically controlled drug release Electrochemistry Communications, 11, 1956, 2009.

169. C. Gouveia-Caridade, C.M.A. Brett, Strategies, development and applications of polymer-modified electrodes for stripping analysis Current Analytical Chemistry, 4, 206, 2008.

170. Sengodu, P; Deshmukh, AD Conducting polymers and their inorganic composites for advanced Li-ion batteries: a review, Por: (Deshmukh, Abhay D.), RSC ADVANCES 5, 52, 42109-42130, 2015.

171. AISalhi, MS, Alam, J, Dass, LA, Raja, M, Recent Advances in Conjugated Polymers for Light Emitting Devices, INTERNATIONAL JOURNAL OF MOLECULAR SCIENCES, 2011; 12(3): 2036-2054, 2011

172. Aznar-Cervantes, Salvador, Roca, Maria I, Martinez, Jose G, Meseguer-Olmo, Luis, Fabrication of conductive electrospun silk fibroin scaffolds by coating with polypyrrole for biomedical applications, Bioelectrochemistry 85, 36-43, 2012. 
173. T.F. Otero, J.G. Martinez, J. Arias-Pardilla, Biomimetic electrochemistry from conducting polymers. A review Artificial muscles, smart membranes, smart drug delivery and computer/neuron interfaces, Electrochimica Acta 84 -112-128, 2012

174. D.T. Simon, S. Kurup, K.C. Larsson, R. Hori, K. Tybrandt, M. Goiny, E.H. Jager, M. Berggren, B. Canlon, A. Richter-Dahlfors, Organic electronics for precise delivery of neurotransmitters to modulate mammalian sensory function Natural. Materials., 8, 742, 2009.

175. Naka. Y.; Fuchiwaki. M.; Tanaka. K. A., micropump driven by a polypyrrolebased conducting polymer soft actuator, Polymer International, 59(3), 352-356, March 2010.

176. www.micromuscle.com, 2011.

177. www.artificialmuscle.com, 2011.

178. www.polypower.com, 2011.

179. www.empa.ch, 2011.

180. www.environmentalrobots.com, 2012.

181. Surgical Correction of Ptosis by Polymeric Artificial Muscles", CIP for US patent Patent No. 7, 625, 404, US Patent Office, Patent Publication No. 20100042146A1, February 18, 2010.

182. Richard Guy Compton (Author), Christopher BatchelorMcauley (Contributor), Edmund J F Dickinson (Contributor), Understanding Voltammetry: Problems and Solutions, ISBN-13: 978-1848167315, Imperial College Press 2012.

183. Allen J. Bard, Larry R. Faulkner, Electrochemical Methods: Fundamentals and Application,John Wiley and sons, 2nd Edition, 2001

184. Rudolf Holze, Experimental Electrochemistry, Wiley- VCH, 2009

185. T.F. Otero, J.M. Sansiñena, Artificial muscles based on conducting polymers, Bioelectrochemistry and Bioenergetics 38, 411-414, 1995.

186. Terje A. Skotheim,John Reynold, Conjugated Polymers: Processing and Applications, CRC Press, 2007.

187. T.F. Otero, M.T. Cortes, Electrochemical characterization and control triplelayer muscles, Smart Structures and Materials 2000: Electroactive Polymer Actuators and Devices (EAPAD) 3987, 252-260, 2000.

188. J.M.Sansiñena, Músculos artificiales: Dispositivos electroquimio-mecánicos basados en músculos artificiales, Tesis Doctoral, Universidad del Pais Vasco, 1998.

189. L.Valero, J.Arias-Pardilla, J.Cauich-Rodriguez, M.A.Smit, T.F.Otero, Polypyrrole free-standing electrodes sense temperature or current during reaction, Polymer International 59, 337-342, 2010. 
190. T.F. Otero, M.J. Ariza, Revisiting the electrochemical and polymeric behavior of a polypyrrole free-standing electrode in aqueous solution, Journal of Physical Chemistry B 107, 13954-13961, 2003.

191. J.Arias-Pardilla, C.Plesse, A.Khaldi, F.Vidal, C.Chevrot, T.F.Otero, Selfsupported semi-interpenetrating polymer networks as reactive ambient sensors, Journal of Electranalytical Chemistry 652, 37-43, 2011.

192. R. Verdu, R. Berenguer, J. Morales, G. Vazquez, T.F. Otero, L. Weruaga, Mechanical characterization of the life cycle of artificial muscles through stereoscopic computer vision and active contours, in: 2005 Int. Conf. Image Process. ICIP Vols 1-5, IEEE, New York, 3705-3708, 2005

193. T.F.Otero, Biomimetic conducting polymers: synthesis, materials, properties, functions, and devices. Polymer Reviews 53, 311-351, 2003.

194. West BJ, Otero TF, Shapiro B, Smela E, Chronoamperometric study of conformational relaxation in PPy(DBS). Journal of Physical Chemistry B 113, 12771293, 2009

195. Wernet W, Monkenbusch M, Wegner G, On structure and properties of polypyrrole alkyl-sulf(on)ates. Molecular Crystals and Liquid Crystals 118, 193-197, 1985.

196. Wernet W, Monkenbusch M, Wegner G A new series of conducting polymers with layered structure-polypyrrole normal-alkylsulfates and normal-alkylsulfonates. Makromolekulare Chemie Rapid Communications 5, 157-164, 1984.

197. David L. Brock, Review of Artificial Muscle based on Contractile Polymers, Massachusetts Institute of Technology Artificial Intelligence Laboratory,A.I.Memo No. 1330, 1991

198. J.Arias, J.G.Martínez, L.Valero, J.Cauich-Rodriguez, M.A.Smit, T.F.Otero, Reactive Polypyrrole Films Sense Environmental Conditions, Actuators 2010,12Th International Conference on new actuators I, 802-806, 2010.

199. T.F.Otero, G.Vázquez, L.Valero, Conducting Polymers as simultaneous sensors-actuators, Proc. of SPIE Electroactive Polymer Actuators and devices 7642, 76420W-1-76420W-12, 2010.

200. Toribio F. Otero, Mercedes Alfaro, Venancio Martinez,. Maria A. Perez and Jose G. Martinez, Biomimetic Structural Electrochemistry from Conducting Polymers: Processes, Charges, and Energies. Coulovoltammetric Results from Films on Metals Revisited, Advanced Functional Materials, 23(31), 3929-3940, 2013.

201. Toribio F. Otero, Jose G. Martinez, Masaki Fuchiwaki, Laura Valero, Structural Electrochemistry from Freestanding Polypyrrole Films: Full Hydrogen Inhibition from Aqueous Solutions, Advanced Functional Materials 24, 1259-1274,2014.

202. T.F. Otero and E. Angulo, Redox behavior od thin polypyrrole filmsoptimatization of response times, Solid State Ionics 63-5, 803-809, 1993. 
203. T.F. Otero, H. Grande, J. Rodriguez, Role of conformational relaxation on the voltammetric behavior of polypyrrole. Experiments and mathematical model, Journal of Physical Chemistry B 101, 8525-8533, 1997.

204. T.F. Otero, J.Rodríguez, H.Grande, Nucleation, relaxation and diffusion during oxid-red processes in conductive polymers, Journal of Brasilian Chemical Society 5 , 179-181, 1994.

205. H. Grande, T.F. Otero, I. Cantero, Conformational relaxation in conducting polymers: effect of polymer-solvent interactions, Journal of Non-Crystalline Solids 235, 619-622, 1998.

206. H. Grande, T.F. Otero, Intrinsic asymmetry, hysteresis, and conformational relaxation during redox switching in polypyrrole: A coulovoltametric study, Journal of Physical Chemistry B 102, 7535-7540, 1998.

207. Hans Jurgen Grande Telleria, Estimulación Electroquímica de la Relajación Conformacional en Polímeros Conductores, 1998.

208. Hans Jurgen Grande Telleria, Modelos de Relajación Electroquímica en Polímeros Conductores, 1994.

209. T.F. Otero, H. Grande, J. Rodriguez, Role of conformational relaxation on the voltammetric behavior of polypyrrole. Experiments and mathematical model, Journal of Physical Chemistry B 101, 8525-8533, 1997.

210. C. Odin, M. Nechtschein, Slow Relaxation in Conducting Polymers, Physical Review Letters 67, 1114-1117, 1991.

211. T.F. Otero, H.J. Grande, J. Rodriguez, Reinterpretation of polypyrrole electrochemistry after consideration of conformational relaxation processes, Journal of Physical Chemistry B 101, 3688-3697, 1997.

212. J. G. Martinez and T. F. Otero, Structural electrochemistry. Chronopotentiometric responses from rising compacted polypyrrole electrodes: experiments and model, RSC Advances 4, 29139-29145, 2014.

213. Terje A. Skotheim, John Reynold, Handbook of Conducting Polymers, 2 Volume Set, 18.3.1.2 CRC, 2007.

214. Robotics-Advances in Research and Application: Schoolarly Edition, 2013 (http. Schoolarlyeditions.com)

215. J.G. Martinez, TF Otero, Biomimetic Dual Sensing-Actuators: Theoretical Description. Sensing Electrolyte Concentration and Driving Current, Journal of Physical Chemistry B 116, 9223-9230, 2012.

215. T.F.Otero, Electro-chemo-mechanical Actuators touching and sensing both, Physical and chemical ambient, Advances in Science and Technology 61, 112-121, 2008. 
216. J.Arias, J.G.Martínez, L.Valero, J.Cauich-Rodriguez, M.A.Smit, T.F.Otero, Reactive Polypyrrole Films Sense Environmental Conditions, Actuators 2010, 12Th International Conference on new actuators I, 802-806, 2010

217. T. F. Otero, J. G. Martinez, Artificial Muscles: A Tool to Quantify Exchanged Solvent during Biomimetic Reactions, Chemistry of Materials, 24, 4093-4099, 2012

218. D. R. Lide, CRC Handbook of Chemistry and Physics, CRC-Press, London, 2002.

219. M. J. M. Jafeen, M. A. Careem, S. Skaarup, A novel method for the determination of membrane hydration numbers of cations in conducting polymers, Journal of Solid State Electrochemistry, 16, 1753-1759, 2012.

220. S. Skaarup, M. J. M. Jafeen, M. A. Careem, Dtermination of membrane hydrations numbers of alkali metal ions by insertion ina conducting polymer, Solid State lonics, 181, 1245-1250, 2010.

221. J.Arias, J.G.Martínez, L.Valero, J.Cauich-Rodriguez, M.A.Smit, T.F.Otero, Reactive Polypyrrole Films Sense Environmental Conditions, Actuators 2010,12Th International Conference on new actuators I, 802-806, 2010.

222. Lee $\mathrm{H}-\mathrm{H}$, Yamaoka S, Murayama N, Shibata J, Dispersion of $\mathrm{Fe} 3 \mathrm{O} 4$ suspensions using sodium dodecylbenzene sulphonate as dispersant. Materials Letters 61:3974-3977, 2007

223. Gaoyi Han, Gaoquan Shi, Conducting polymer electrochemical actuator made of high-strength three-layered composite films of polythiophene and polypyrrole, Sensors and Actuators B: Chemical 99,525-531, 2004.

224. Q. Yao, G. Alici, G. A. Spinks, Feedback control of tri-layer polymer actuators to improve their positioning ability and speed of response

Sensors and Actuators A-Physical, 144, 176, 2008.

225. Challa S. S. R. Kuma, Biomimetic and Bioinspired Nanomaterials, WileyVCH,2010. 